EUROPEAN ORGANIZATION FOR NUCLEAR RESEARCH

CERN-PH-EP/2005-045

4 October 2005

\title{
Measurement and Interpretation of Fermion-Pair Production at LEP energies above the $\mathrm{Z}$ Resonance
}

DELPHI Collaboration

\begin{abstract}
This paper presents DELPHI measurements and interpretations of crosssections, forward-backward asymmetries, and angular distributions, for the $\mathrm{e}^{+} \mathrm{e}^{-} \rightarrow \mathrm{f} \overline{\mathrm{f}}$ process for centre-of-mass energies above the $\mathrm{Z}$ resonance, from $\sqrt{s} \sim 130-207 \mathrm{GeV}$ at the LEP collider.

The measurements are consistent with the predictions of the Standard Model and are used to study a variety of models including the S-Matrix ansatz for $\mathrm{e}^{+} \mathrm{e}^{-} \rightarrow \mathrm{f} \overline{\mathrm{f}}$ scattering and several models which include physics beyond the Standard Model: the exchange of $\mathrm{Z}^{\prime}$ bosons, contact interactions between fermions, the exchange of gravitons in large extra dimensions and the exchange of $\tilde{\nu}$ in R-parity violating supersymmetry.
\end{abstract}

This paper is dedicated to the memory of Alan Segar. 


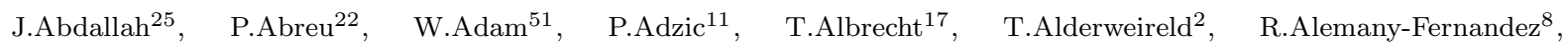
T.Allmendinger ${ }^{17}$, P.P.Allport ${ }^{23}$, U.Amaldi ${ }^{29}$, N.Amapane ${ }^{45}$, S.Amato ${ }^{48}$, E.Anashkin ${ }^{36}$, A.Andreazza ${ }^{28}$, S.Andringa ${ }^{22}$, N.Anjos ${ }^{22}$, P.Antilogus ${ }^{25}$, W-D.Apel ${ }^{17}$, Y.Arnoud ${ }^{14}$, S.Ask ${ }^{26}$, B.Asman ${ }^{44}$, J.E.Augustin ${ }^{25}$, A.Augustinus ${ }^{8}$, P.Baillon ${ }^{8}$, A.Ballestrero ${ }^{46}$, P.Bambade ${ }^{20}$, R.Barbier ${ }^{27}$, D.Bardin ${ }^{16}$, G.J.Barker ${ }^{17}$, A.Baroncelli ${ }^{39}$, M.Battaglia ${ }^{8}$, M.Baubillier ${ }^{25}$, K-H.Becks ${ }^{53}$, M.Begalli ${ }^{6}$, A.Behrmann ${ }^{53}$, E.Ben-Haim ${ }^{20}$, N.Benekos ${ }^{32}$, A.Benvenuti ${ }^{5}$, C.Berat ${ }^{14}$, M.Berggren ${ }^{25}$, L.Berntzon $^{44}$, D.Bertrand ${ }^{2}$, M.Besancon ${ }^{40}$, N.Besson ${ }^{40}$, D.Bloch ${ }^{9}$, M.Blom ${ }^{31}$, M.Bluj ${ }^{52}$, M.Bonesini ${ }^{29}$, M.Boonekamp ${ }^{40}$, P.S.L.Booth ${ }^{\dagger 23}$, G.Borisov ${ }^{21}$, O.Botner ${ }^{49}$, B.Bouquet ${ }^{20}$, T.J.V.Bowcock ${ }^{23}$, I.Boyko ${ }^{16}$, M.Bracko ${ }^{43}$, R.Brenner ${ }^{49}$, E.Brodet ${ }^{35}$, P.Bruckman ${ }^{18}$, J.M.Brunet ${ }^{7}$, P.Buschmann ${ }^{53}$, M.Calvi ${ }^{29}$, T.Camporesi ${ }^{8}$, V.Canale ${ }^{38}$, F.Carena ${ }^{8}$, N.Castro $^{22}$, F.Cavallo ${ }^{5}$, M.Chapkin ${ }^{42}$, Ph.Charpentier ${ }^{8}$, P.Checchia ${ }^{36}$, R.Chierici ${ }^{8}$, P.Chliapnikov ${ }^{42}$, J.Chudoba ${ }^{8}$, S.U.Chung ${ }^{8}$, K.Cieslik ${ }^{18}$, P.Collins ${ }^{8}$, R.Contri ${ }^{13}$, G.Cosme ${ }^{20}$, F.Cossutti ${ }^{47}$, M.J.Costa ${ }^{50}$, D.Crennell ${ }^{37}$, J.Cuevas ${ }^{34}$, J.D'Hondt $^{2}$, J.Dalmau ${ }^{44}$, T.da Silva ${ }^{48}$, W.Da Silva ${ }^{25}$, G.Della Ricca ${ }^{47}$, A.De Angelis ${ }^{47}$, W.De Boer ${ }^{17}$, C.De Clercq ${ }^{2}$, B.De Lotto ${ }^{47}$, N.De Maria ${ }^{45}$, A.De Min ${ }^{36}$, L.de Paula ${ }^{48}$, L.Di Ciaccio ${ }^{38}$, A.Di Simone ${ }^{39}$, K.Doroba ${ }^{52}$, J.Drees ${ }^{53,8}$, G.Eigen $^{4}$, T.Ekelof ${ }^{49}$, M.Ellert ${ }^{49}$, M.Elsing ${ }^{8}$, M.C.Espirito Santo ${ }^{22}$, G.Fanourakis ${ }^{11}$, D.Fassouliotis ${ }^{11,3}$, M.Feindt ${ }^{17}$,

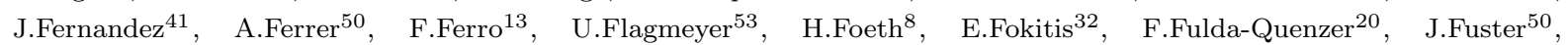
M.Gandelman ${ }^{48}$, C.Garcia ${ }^{50}$, Ph.Gavillet ${ }^{8}$, E.Gazis ${ }^{32}$, R.Gokieli ${ }^{8,52}$, B.Golob ${ }^{43}$, G.Gomez-Ceballos ${ }^{41}$, P.Goncalves ${ }^{22}$, E.Graziani ${ }^{39}$, G.Grosdidier ${ }^{20}$, K.Grzelak ${ }^{52}$, J.Guy ${ }^{37}$, C.Haag ${ }^{17}$, A.Hallgren ${ }^{49}$, K.Hamacher ${ }^{53}$, K.Hamilton ${ }^{35}$, S.Haug ${ }^{33}$, F.Hauler ${ }^{17}$, V.Hedberg ${ }^{26}$, M.Hennecke ${ }^{17}$, H.Herr ${ }^{\dagger 8}$, J.Hoffman ${ }^{52}$, S-O.Holmgren ${ }^{44}$, P.J.Holt ${ }^{8}$, M.A.Houlden ${ }^{23}$, K.Hultqvist ${ }^{44}$, J.N.Jackson ${ }^{23}$, G.Jarlskog ${ }^{26}$, P.Jarry ${ }^{40}$, D.Jeans ${ }^{35}$, E.K.Johansson ${ }^{44}$, P.D.Johansson ${ }^{44}$, P.Jonsson ${ }^{27}$, C.Joram ${ }^{8}$, L.Jungermann ${ }^{17}$, F.Kapusta ${ }^{25}$, S.Katsanevas ${ }^{27}$, E.Katsoufis ${ }^{32}$, G.Kernel ${ }^{43}$, B.P.Kersevan ${ }^{8,43}$, U.Kerzel ${ }^{17}$, B.T.King ${ }^{23}$, N.J.Kjaer ${ }^{8}$, P.Kluit ${ }^{31}$, P.Kokkinias ${ }^{11}$, C.Kourkoumelis ${ }^{3}$, O.Kouznetsov ${ }^{16}$, Z.Krumstein ${ }^{16}$, M.Kucharczyk ${ }^{18}$, J.Lamsa $^{1}$, G.Leder ${ }^{51}$, F.Ledroit ${ }^{14}$, L.Leinonen ${ }^{44}$, R.Leitner ${ }^{30}$, J.Lemonne ${ }^{2}$, V.Lepeltier ${ }^{20}$, T.Lesiak ${ }^{18}$, W.Liebig ${ }^{53}$, D.Liko ${ }^{51}$, A.Lipniacka ${ }^{44}$, J.H.Lopes ${ }^{48}$, J.M.Lopez ${ }^{34}$, D.Loukas ${ }^{11}$, P.Lutz ${ }^{40}$, L.Lyons ${ }^{35}$, J.MacNaughton ${ }^{51}$, A.Malek ${ }^{53}$, S.Maltezos $^{32}$, F.Mandl ${ }^{51}$, J.Marco ${ }^{41}$, R.Marco ${ }^{41}$, B.Marechal ${ }^{48}$, M.Margoni ${ }^{36}$, J-C.Marin ${ }^{8}$, C.Mariotti ${ }^{8}$, A.Markou ${ }^{11}$, C.Martinez-Rivero $^{41}$, J.Masik ${ }^{12}$, N.Mastroyiannopoulos ${ }^{11}$, F.Matorras ${ }^{41}$, $\quad$ C.Matteuzzi $^{29}$, F.Mazzucato ${ }^{36}$, M.Mazzucato $^{36}$, R.Mc Nulty ${ }^{23}$, C.Meroni ${ }^{28}$, E.Migliore ${ }^{45}$, W.Mitaroff ${ }^{51}$, U.Mjoernmark ${ }^{26}$, T.Moa ${ }^{44}$, M.Moch $^{17}$, K.Moenig ${ }^{8,10}$, R.Monge ${ }^{13}$, J.Montenegro ${ }^{31}$, D.Moraes ${ }^{48}$, S.Moreno ${ }^{22}$, P.Morettini ${ }^{13}$, U.Mueller ${ }^{53}$, K.Muenich ${ }^{53}$, M.Mulders ${ }^{31}$, L.Mundim ${ }^{6}$, W.Murray ${ }^{37}$, B.Muryn ${ }^{19}$, G.Myatt ${ }^{35}$, T.Myklebust ${ }^{33}$, M.Nassiakou ${ }^{11}$, F.Navarria ${ }^{5}$, K.Nawrocki ${ }^{52}$, R.Nicolaidou ${ }^{40}$, V.Nikolaenko ${ }^{9}$, M.Nikolenko ${ }^{16,9}$, A.Oblakowska-Mucha ${ }^{19}$, V.Obraztsov ${ }^{42}$, A.Olshevski ${ }^{16}$, A.Onofre ${ }^{22}$, R.Orava ${ }^{15}$, K.Osterberg ${ }^{15}$, A.Ouraou ${ }^{40}$, A.Oyanguren ${ }^{50}$, M.Paganoni ${ }^{29}$, S.Paiano ${ }^{5}$, J.P.Palacios ${ }^{23}$, H.Palka ${ }^{18}$, Th.D.Papadopoulou ${ }^{32}$, L.Pape ${ }^{8}$, C.Parkes ${ }^{24}$, F.Parodi ${ }^{13}$, U.Parzefall ${ }^{8}$, A.Passeri ${ }^{39}$, O.Passon ${ }^{53}$, L.Peralta ${ }^{22}$, V.Perepelitsa ${ }^{50}$, A.Perrotta ${ }^{5}$, A.Petrolini ${ }^{13}$, J.Piedra ${ }^{41}$, L.Pieri ${ }^{39}$, F.Pierre ${ }^{40}$, M.Pimenta ${ }^{22}$, E.Piotto ${ }^{8}$, T.Podobnik ${ }^{43}$, V.Poireau ${ }^{8}$, M.E.Pol ${ }^{6}$, G.Polok ${ }^{18}$, V.Pozdniakov ${ }^{16}$, N.Pukhaeva ${ }^{2,16}$, A.Pullia ${ }^{29}$, J.Rames ${ }^{12}$, A.Read ${ }^{33}$, P.Rebechi ${ }^{8}$, J.Rehn ${ }^{17}$, D.Reid ${ }^{31}$, R.Reinhardt ${ }^{53}$, P.Renton ${ }^{35}$, F.Richard ${ }^{20}$, J.Ridky ${ }^{12}$, M.Rivero ${ }^{41}$, D.Rodriguez ${ }^{41}$, A.Romero ${ }^{45}$, P.Ronchese $^{36}$, P.Roudeau ${ }^{20}$, T.Rovelli ${ }^{5}$, V.Ruhlmann-Kleider ${ }^{40}$, D.Ryabtchikov ${ }^{42}$, A.Sadovsky ${ }^{16}$, L.Salmi ${ }^{15}$, J.Salt ${ }^{50}$, C.Sander ${ }^{17}$, A.Savoy-Navarro ${ }^{25}$, U.Schwickerath ${ }^{8}$, A.Segar ${ }^{\dagger 5}$, R.Sekulin ${ }^{37}$, M.Siebel ${ }^{53}$, A.Sisakian ${ }^{16}$, G.Smadja ${ }^{27}$, O.Smirnova ${ }^{26}$, A.Sokolov ${ }^{42}$, A.Sopczak ${ }^{21}$, R.Sosnowski ${ }^{52}$, T.Spassov ${ }^{8}$, M.Stanitzki ${ }^{17}$, A.Stocchi ${ }^{20}$, J.Strauss ${ }^{51}$, B.Stugu ${ }^{4}$, M.Szczekowski ${ }^{52}$, M.Szeptycka ${ }^{52}$, T.Szumlak ${ }^{19}$, T.Tabarelli ${ }^{29}$, A.C.Taffard ${ }^{23}$, F.Tegenfeldt ${ }^{49}$, J.Timmermans ${ }^{31}$, L.Tkatchev ${ }^{16}$, M.Tobin ${ }^{23}$, S.Todorovova ${ }^{12}$, B.Tome ${ }^{22}$, A.Tonazzo ${ }^{29}$, P.Tortosa ${ }^{50}$, P.Travnicek ${ }^{12}$, D.Treille ${ }^{8}$, G.Tristram ${ }^{7}$, M.Trochimczuk ${ }^{52}$, C.Troncon ${ }^{28}$, M-L.Turluer ${ }^{40}$, I.A.Tyapkin ${ }^{16}$, P.Tyapkin ${ }^{16}$, S.Tzamarias ${ }^{11}$, V.Uvarov ${ }^{42}$, G.Valenti ${ }^{5}$, P.Van Dam ${ }^{31}$, J.Van Eldik ${ }^{8}$, N.van Remortel ${ }^{15}$, I.Van Vulpen ${ }^{8}$, G.Vegni ${ }^{28}$, F.Veloso ${ }^{22}$, W.Venus ${ }^{37}$, P.Verdier ${ }^{27}$, V.Verzi $^{38}$, D.Vilanova ${ }^{40}$, L.Vitale ${ }^{47}$, V.Vrba ${ }^{12}$, H.Wahlen ${ }^{53}$, A.J.Washbrook ${ }^{23}$, C.Weiser ${ }^{17}$, D.Wicke ${ }^{8}$, J.Wickens ${ }^{2}$, G.Wilkinson ${ }^{35}$, M.Winter ${ }^{9}$, M.Witek ${ }^{18}$, O.Yushchenko ${ }^{42}$, A.Zalewska ${ }^{18}$, P.Zalewski ${ }^{52}$, D.Zavrtanik ${ }^{43}$, V.Zhuravlov ${ }^{16}$, N.I.Zimin ${ }^{16}$, A.Zintchenko ${ }^{16}$, M.Zupan ${ }^{11}$ 


\footnotetext{
${ }^{1}$ Department of Physics and Astronomy, Iowa State University, Ames IA 50011-3160, USA

${ }^{2}$ Physics Department, Universiteit Antwerpen, Universiteitsplein 1, B-2610 Antwerpen, Belgium and IIHE, ULB-VUB, Pleinlaan 2, B-1050 Brussels, Belgium

and Faculté des Sciences, Univ. de l'Etat Mons, Av. Maistriau 19, B-7000 Mons, Belgium

${ }^{3}$ Physics Laboratory, University of Athens, Solonos Str. 104, GR-10680 Athens, Greece

${ }^{4}$ Department of Physics, University of Bergen, Allégaten 55, NO-5007 Bergen, Norway

${ }^{5}$ Dipartimento di Fisica, Università di Bologna and INFN, Via Irnerio 46, IT-40126 Bologna, Italy

${ }^{6}$ Centro Brasileiro de Pesquisas Físicas, rua Xavier Sigaud 150, BR-22290 Rio de Janeiro, Brazil and Depto. de Física, Pont. Univ. Católica, C.P. 38071 BR-22453 Rio de Janeiro, Brazil and Inst. de Física, Univ. Estadual do Rio de Janeiro, rua São Francisco Xavier 524, Rio de Janeiro, Brazil ${ }^{7}$ Collège de France, Lab. de Physique Corpusculaire, IN2P3-CNRS, FR-75231 Paris Cedex 05, France ${ }^{8}$ CERN, CH-1211 Geneva 23, Switzerland

${ }^{9}$ Institut de Recherches Subatomiques, IN2P3 - CNRS/ULP - BP20, FR-67037 Strasbourg Cedex, France

${ }^{10}$ Now at DESY-Zeuthen, Platanenallee 6, D-15735 Zeuthen, Germany

${ }^{11}$ Institute of Nuclear Physics, N.C.S.R. Demokritos, P.O. Box 60228, GR-15310 Athens, Greece

${ }^{12} \mathrm{FZU}$, Inst. of Phys. of the C.A.S. High Energy Physics Division, Na Slovance 2, CZ-180 40, Praha 8, Czech Republic

${ }^{13}$ Dipartimento di Fisica, Università di Genova and INFN, Via Dodecaneso 33, IT-16146 Genova, Italy

${ }^{14}$ Institut des Sciences Nucléaires, IN2P3-CNRS, Université de Grenoble 1, FR-38026 Grenoble Cedex, France

${ }^{15}$ Helsinki Institute of Physics and Department of Physical Sciences, P.O. Box 64, FIN-00014 University of Helsinki, Finland

${ }^{16}$ Joint Institute for Nuclear Research, Dubna, Head Post Office, P.O. Box 79, RU-101 000 Moscow, Russian Federation

${ }^{17}$ Institut für Experimentelle Kernphysik, Universität Karlsruhe, Postfach 6980, DE-76128 Karlsruhe, Germany

${ }^{18}$ Institute of Nuclear Physics PAN,Ul. Radzikowskiego 152, PL-31142 Krakow, Poland

${ }^{19}$ Faculty of Physics and Nuclear Techniques, University of Mining and Metallurgy, PL-30055 Krakow, Poland

${ }^{20}$ Université de Paris-Sud, Lab. de l'Accélérateur Linéaire, IN2P3-CNRS, Bât. 200, FR-91405 Orsay Cedex, France

${ }^{21}$ School of Physics and Chemistry, University of Lancaster, Lancaster LA1 4YB, UK

${ }^{22}$ LIP, IST, FCUL - Av. Elias Garcia, 14-1 ${ }^{\circ}$, PT-1000 Lisboa Codex, Portugal

${ }^{23}$ Department of Physics, University of Liverpool, P.O. Box 147, Liverpool L69 3BX, UK

${ }^{24}$ Dept. of Physics and Astronomy, Kelvin Building, University of Glasgow, Glasgow G12 8QQ

${ }^{25}$ LPNHE, IN2P3-CNRS, Univ. Paris VI et VII, Tour 33 (RdC), 4 place Jussieu, FR-75252 Paris Cedex 05, France

${ }^{26}$ Department of Physics, University of Lund, Sölvegatan 14, SE-223 63 Lund, Sweden

${ }^{27}$ Université Claude Bernard de Lyon, IPNL, IN2P3-CNRS, FR-69622 Villeurbanne Cedex, France

${ }^{28}$ Dipartimento di Fisica, Università di Milano and INFN-MILANO, Via Celoria 16, IT-20133 Milan, Italy

${ }^{29}$ Dipartimento di Fisica, Univ. di Milano-Bicocca and INFN-MILANO, Piazza della Scienza 2, IT-20126 Milan, Italy

${ }^{30}$ IPNP of MFF, Charles Univ., Areal MFF, V Holesovickach 2, CZ-180 00, Praha 8, Czech Republic

${ }^{31}$ NIKHEF, Postbus 41882, NL-1009 DB Amsterdam, The Netherlands

${ }^{32}$ National Technical University, Physics Department, Zografou Campus, GR-15773 Athens, Greece

${ }^{33}$ Physics Department, University of Oslo, Blindern, NO-0316 Oslo, Norway

${ }^{34}$ Dpto. Fisica, Univ. Oviedo, Avda. Calvo Sotelo s/n, ES-33007 Oviedo, Spain

${ }^{35}$ Department of Physics, University of Oxford, Keble Road, Oxford OX1 3RH, UK

${ }^{36}$ Dipartimento di Fisica, Università di Padova and INFN, Via Marzolo 8, IT-35131 Padua, Italy

${ }^{37}$ Rutherford Appleton Laboratory, Chilton, Didcot OX11 OQX, UK

${ }^{38}$ Dipartimento di Fisica, Università di Roma II and INFN, Tor Vergata, IT-00173 Rome, Italy

${ }^{39}$ Dipartimento di Fisica, Università di Roma III and INFN, Via della Vasca Navale 84, IT-00146 Rome, Italy

${ }^{40}$ DAPNIA/Service de Physique des Particules, CEA-Saclay, FR-91191 Gif-sur-Yvette Cedex, France

${ }^{41}$ Instituto de Fisica de Cantabria (CSIC-UC), Avda. los Castros s/n, ES-39006 Santander, Spain

${ }^{42}$ Inst. for High Energy Physics, Serpukov P.O. Box 35, Protvino, (Moscow Region), Russian Federation

${ }^{43}$ J. Stefan Institute, Jamova 39, SI-1000 Ljubljana, Slovenia and Laboratory for Astroparticle Physics,

Nova Gorica Polytechnic, Kostanjeviska 16a, SI-5000 Nova Gorica, Slovenia,

and Department of Physics, University of Ljubljana, SI-1000 Ljubljana, Slovenia

${ }^{44}$ Fysikum, Stockholm University, Box 6730, SE-113 85 Stockholm, Sweden

${ }^{45}$ Dipartimento di Fisica Sperimentale, Università di Torino and INFN, Via P. Giuria 1, IT-10125 Turin, Italy

${ }^{46}$ INFN,Sezione di Torino and Dipartimento di Fisica Teorica, Università di Torino, Via Giuria 1, IT-10125 Turin, Italy

${ }^{47}$ Dipartimento di Fisica, Università di Trieste and INFN, Via A. Valerio 2, IT-34127 Trieste, Italy and Istituto di Fisica, Università di Udine, IT-33100 Udine, Italy

${ }^{48}$ Univ. Federal do Rio de Janeiro, C.P. 68528 Cidade Univ., Ilha do Fundão BR-21945-970 Rio de Janeiro, Brazil

${ }^{49}$ Department of Radiation Sciences, University of Uppsala, P.O. Box 535, SE-751 21 Uppsala, Sweden

${ }^{50}$ IFIC, Valencia-CSIC, and D.F.A.M.N., U. de Valencia, Avda. Dr. Moliner 50, ES-46100 Burjassot (Valencia), Spain

${ }^{51}$ Institut für Hochenergiephysik, Österr. Akad. d. Wissensch., Nikolsdorfergasse 18, AT-1050 Vienna, Austria

${ }^{52}$ Inst. Nuclear Studies and University of Warsaw, Ul. Hoza 69, PL-00681 Warsaw, Poland

${ }^{53}$ Fachbereich Physik, University of Wuppertal, Postfach 100 127, DE-42097 Wuppertal, Germany

$\dagger$ deceased
} 


\section{Introduction}

This paper presents measurements and interpretations of cross-sections, $\sigma$, forwardbackward asymmetries, $\mathrm{A}_{\mathrm{FB}}$, and angular distributions, $\mathrm{d} \sigma / \mathrm{d} \cos \theta$, for $\mathrm{e}^{+} \mathrm{e}^{-} \rightarrow \mathrm{f} \overline{\mathrm{f}}$ processes for centre-of-mass energies above the $\mathrm{Z}$ resonance, as measured in the DELPHI experiment at the LEP collider. Measurements of flavour tagged q $\bar{q}$ samples will be included in an additional publication.

For the first part of the LEP programme, LEP I, $\mathrm{e}^{+} \mathrm{e}^{-}$collisions were provided at centre-of-mass energies close to the $Z$ resonance. Measurements of the process $\mathrm{e}^{+} \mathrm{e}^{-} \rightarrow \mathrm{f} \overline{\mathrm{f}}$ were used to determine properties of the $\mathrm{Z}$ and electroweak parameters of the Standard Model [1,2]. In 1995 the operation of LEP moved into the LEP II programme. The collision energy was raised to significantly above the $\mathrm{Z}$ resonance, and a total of approximately $0.7 \mathrm{fb}^{-1}$ of integrated luminosity was delivered to the DELPHI experiment at energies ranging from $130 \mathrm{GeV}$ to a maximum collision energy of $209 \mathrm{GeV}$ during LEP II operations. A breakdown of the centre-of-mass energies, and integrated luminosities is given in Table 1. Measurements of the process $\mathrm{e}^{+} \mathrm{e}^{-} \rightarrow \mathrm{f} \overline{\mathrm{f}}$ from LEP II are less sensitive to the electroweak parameters of the Standard Model. Nevertheless, taken together, the measurements constitute a test of the Standard Model at the $\mathcal{O}(1 \%)$ level, at the highest $\mathrm{e}^{+} \mathrm{e}^{-}$collision energies to date. Furthermore, the $\mathrm{e}^{+} \mathrm{e}^{-} \rightarrow \mathrm{f} \overline{\mathrm{f}}$ measurements at LEP II are predicted to be more sensitive to a variety of models of physics beyond the Standard Model than the LEP I measurements. Having determined many of the parameters of the Standard Model, largely from LEP I data, studies of the process $\mathrm{e}^{+} \mathrm{e}^{-} \rightarrow \mathrm{f} \overline{\mathrm{f}}$ at LEP II are, therefore, a suitable place to look for physics beyond the Standard Model.

Results from the analysis of data at centre-of-mass energies from 130-189 GeV have already been published [3,4]. Results at energies from $192-207 \mathrm{GeV}$ are published here for the first time. Some previously published results at energies of 183 and $189 \mathrm{GeV}$ [4] have been reanalysed and are presented again here. For completeness we present again the results from $130-172 \mathrm{GeV}$ data which have not been reanalysed. Results on f $\bar{f}$ production from the other LEP experiments can be found in [5-7].

The operation of LEP during the LEP II programme is discussed in Section 2. The DELPHI detector itself is described in Section 3, and the measurement of the integrated luminosity is described in Section 4. Features of $\mathrm{f} \overline{\mathrm{f}}$ production at LEP II, details of the event simulation and theoretical predictions are given in Section 5. Sections 5.1 to 5.4 cover the analysis of the individual channels $\mathrm{e}^{+} \mathrm{e}^{-} \rightarrow \mathrm{e}^{+} \mathrm{e}^{-}, \mathrm{e}^{+} \mathrm{e}^{-} \rightarrow \mu^{+} \mu^{-}, \mathrm{e}^{+} \mathrm{e}^{-} \rightarrow \tau^{+} \tau^{-}$ and $\mathrm{e}^{+} \mathrm{e}^{-} \rightarrow \mathrm{q} \overline{\mathrm{q}}$. In each section the criteria for selecting events are described and the methods for evaluating the efficiency and backgrounds are discussed. Results of the individual measurements are provided and the principal sources of systematic error are described. The sets of measurements are summarised in Section 5.5, where the results are compared to the predictions of the Standard Model. Further interpretations of the results are presented in Section 6. In particular the results, together with LEP I data, are interpreted with the S-Matrix formalism in Section 6.1, and also in a variety of models which include explicit forms of physics beyond the Standard Model: models with $\mathrm{Z}^{\prime}$ bosons in Section 6.2, contact interactions (Section 6.3), models which include the exchange of gravitons in large extra dimensions (Section 6.4) and models which consider possible $s$ or $t$ channel sneutrino $\widetilde{\nu}_{l}$ exchange in R-parity violating supersymmetry (Section 6.5). In each case, a resumé of the model, the method of comparing predictions of the model to the data and the results of the interpretation are provided. Conclusions drawn from the DELPHI analyses of the $\mathrm{e}^{+} \mathrm{e}^{-} \rightarrow \mathrm{f} \overline{\mathrm{f}}$ processes at LEP II are given in Section 7 . 


\begin{tabular}{|c|c|c|c|}
\hline Year & $\begin{array}{c}\text { Nominal } \\
\text { Energy } \\
(\mathrm{GeV})\end{array}$ & $\begin{array}{c}\text { Mean } \\
\text { Energy } \\
(\mathrm{GeV})\end{array}$ & $\begin{array}{c}\text { Integrated } \\
\text { Luminosity } \\
\left(\mathrm{pb}^{-1}\right)\end{array}$ \\
\hline \hline 1995 & 130 & 130.3 & 3 \\
136 & 136.3 & 3 \\
\hline 1996 & 161 & 161.5 & 10 \\
& 172 & 172.4 & 10 \\
\hline 1997 & 183 & 182.7 & 53 \\
\hline 1998 & 189 & 188.6 & 155 \\
\hline 1999 & 192 & 191.8 & 25 \\
& 196 & 195.7 & 76 \\
& 200 & 199.7 & 83 \\
& 202 & 201.8 & 40 \\
\hline 2000 & 205 & 204.9 & 82 \\
& 207 & 206.6 & 135 \\
\hline \hline Total & & 195.6 & 675 \\
\hline
\end{tabular}

Table 1: Nominal centre-of-mass energies with approximate centre-of-mass energy and integrated luminosity collected by DELPHI. The values used in the final analyses differ due to data collection efficiencies and selection criteria. 


\section{LEP}

The LEP collider was upgraded from its original configuration, used for running at energies around the $\mathrm{Z}$ resonance, by the addition of superconducting RF cavities. This then allowed operations at energies well above the $\mathrm{Z}$ resonance. In the years 1995 to 1999 LEP delivered $\mathrm{e}^{+} \mathrm{e}^{-}$collisions at one or more discrete energies, each LEP fill corresponding to a particular energy. In 2000 the luminosity at any given time was delivered at the maximum energy available from the LEP RF system, within a margin of safety which allowed for the trip of either two, one or no RF units before the beam was lost. In a given fill the energy would usually be increased from the limit set by two RF trips, to the limit set by one trip and eventually no RF trips. The luminosity was, therefore, delivered more or less continuously over a range of energies. For analysis purposes the data were grouped into two energy points: data taken at centre-of-mass energies between 202.5 and 205.5 GeV and data taken at energies above 205.5 GeV. Data collected during the time in which the energy was increasing are not analysed here, which represents a loss of approximately $1 \%$ of the delivered luminosity.

As well as providing collisions at energies above the $\mathrm{Z}$ resonance, LEP also ran at a centre-of-mass energy close to the pole of the $\mathrm{Z}$ resonance in each year. The data gathered at this energy were used by DELPHI for detector calibration purposes. Typically $2.5 \mathrm{pb}^{-1}$ were delivered at the start of the year in 1996-2000, with additional luminosity delivered on several other occasions when requested by the experiments. The data collected in 1995 at centre-of-mass energies of 130 and $136 \mathrm{GeV}$ followed a long run at energies close to the $\mathrm{Z}$ resonance.

The energy of the $\mathrm{e}^{+} \mathrm{e}^{-}$collisions was determined by the LEP energy group. During LEP I this was based primarily on the resonant depolarisation technique [8], where the energy was determined with very high precision at the end of a large number of fills making the measurements at close to the actual collision energies. The measurements were used to normalise a model of the RF system, from which the energies during actual collisions were determined. At LEP II the energy was again calibrated using the resonant depolarisation technique, but it was not possible to obtain polarisation at the actual collision energies, so the RF model had to be used to extrapolate from the calibration energies (up to $60 \mathrm{GeV}$ per beam) to the collision energies. The accuracy of this extrapolation was checked using a number of techniques:

- dedicated LEP runs, at collision energies, in which the energy of the electron and positron beams were determined from the deflection of the beams in a magnetic spectrometer whose magnetic field was known to high precision;

- measurements of the synchrotron tune as a function of RF voltage;

- measurements of the total magnetic field seen by the beams using the flux loop system of LEP.

The precision that was obtained was $\pm(20-40) \mathrm{MeV}$ on the centre-of-mass energy [9]. The instantaneous difference in energy between the electron and positron beams was less than $\pm 100 \mathrm{MeV}$.

The beam energy spread at LEP II is larger than at LEP I, due to the increased synchrotron energy loss at the higher beam energies. At LEP I the beam energy spread was $\sim 40 \mathrm{MeV}$, at LEP II this increases to $\sim 180 \mathrm{MeV}$ at the highest centre-of-mass energies. At LEP I the beam energy spread had to be taken into account in the estimation of the total cross-section. For example, at the peak of the $\mathrm{Z}$ resonance, the expected cross-section was significantly lower than would have been observed for a monochromatic beam. At LEP II the corresponding correction is not significant for $\mathrm{e}^{+} \mathrm{e}^{-} \rightarrow \mathrm{f} \overline{\mathrm{f}}$ since 
the cross-sections are nearly linear over a small range of energies. However, for certain measurements at LEP II it is necessary to account for the beam energy spread for the determination of the event kinematics.

\section{The DELPHI Detector}

A detailed description of the DELPHI apparatus as used at LEP I and its performance can be found in refs. [10,11]. For the present analysis the following parts of the detector are relevant:

- for the measurement of charged particles the Microvertex Detector (VD), the Inner Detector (ID), the Time Projection Chamber (TPC), the Outer Detector(OD) and the Forward Chambers A and B (FCA and FCB). For the running from 1995 onwards a lengthened Inner Detector was installed. The polar angle ${ }^{1}$ coverage was thereby extended from $23^{\circ}<\theta<157^{\circ}$ to $15^{\circ}<\theta<165^{\circ}$ with a corresponding increase in forward tracking efficiency. For a period in 2000 when part of the TPC was not operational (see later), space points from the Barrel RICH detector were included in the track fit;

- for the measurement of electromagnetic energy the High-density Projection Chamber (HPC) and the Forward Electromagnetic Calorimeter (FEMC); these detectors were also used for identifying minimum ionising particles;

- for the measurement of the hadronic energy and muon identification the Hadron Calorimeter (HCAL), which covered both the barrel and endcap regions;

- for muon identification the barrel (MUB), the endcap (MUF), and the surround muon chambers (SMC), which completed the polar angle coverage between barrel and endcap;

- for the trigger, besides the detectors mentioned above, the barrel Time of Flight counters (TOF), the endcap scintillators (HOF) and a scintillator layer embedded in the HPC;

- for the measurement of luminosity the Small Angle Tile Calorimeter (STIC).

The DELPHI detector was upgraded for LEP II data taking. Changes were made to the subdetectors, the trigger system, the run control and the algorithms used in the offline reconstruction of tracks, which improved the performance compared to LEP I [11].

The major change was the inclusion of the Very Forward Tracker (VFT) [12], which extended the coverage of the innermost silicon tracker out to $11^{\circ}<\theta<169^{\circ}$. Together with improved tracking algorithms and alignment and calibration procedures optimised for LEP II, these changes led to an improved track reconstruction efficiency in the forward regions of DELPHI.

A smaller change was the removal of the tungsten nose cone in front of the Small Angle Tile Calorimeter, to increase the forward electromagnetic coverage. This change had consequences for the luminosity determination discussed in Section 4.

Changes were made to the electronics of the trigger and timing system which improved the stability of the running during data taking [13]. The trigger conditions were optimised for LEP II running, to give high efficiency for Standard Model 2- and 4-fermion processes and also to give sensitivity to events which might be signatures of new physics. In addition, improvements were made to the operation of the detector during the LEP

\footnotetext{
${ }^{1}$ The DELPHI coordinate system is a RH system with the $z$-axis collinear with the incoming electron beam, the $x$ axis pointing to the centre of the LEP accelerator and the $y$ axis vertical. The polar angle $\theta$ is with reference to the $z$-axis, and $\phi$ is the azimuthal angle in the $x, y$ plane. $\mathrm{r}=\sqrt{x^{2}+y^{2}}$.
} 
cycle, to prepare the detector for data taking at the very start of stable collisions of the $\mathrm{e}^{+} \mathrm{e}^{-}$beams, and to respond to adverse backgrounds from LEP, if they occurred. These changes led to an overall improvement in the efficiency for collecting the delivered luminosity from $\sim 85 \%$ at the start of LEP II in 1995 to $\sim 95 \%$ by the end in 2000 .

During the operation of the DELPHI detector in 2000 one of the 12 sectors of the central tracking chamber, the TPC, failed. After September $1^{\text {st }}$ it was not possible to detect tracks left by charged particles inside the broken sector. The data affected corresponds to $\sim 1 / 4$ of that collected in 2000. Nevertheless, the redundancy of the tracking system of DELPHI meant that tracks of charged particles passing through that sector could still be reconstructed from signals in other tracking detectors. A modified track reconstruction algorithm was used in this sector, which included space points reconstructed in the Barrel RICH detector, these points having a significant role in the determination of the polar angle of tracks. As a result, the track reconstruction efficiency was only slightly reduced in the region covered by the broken sector, but on average the resolution on the perigee parameters of the tracks was worse than prior to the failure of the sector. The impact of the failure of this part of the detector on the different analyses is discussed further in Section 5 .

\section{Luminosity determination}

The luminosity measurement followed closely the analysis described in [2]. It was based on counting the number of Bhabha events in the Small Angle Tile Calorimeter of DELPHI, which covered the region between 29 and $185 \mathrm{mrad}$ with respect to the beam line. A detailed description of this detector and its performance can be found in [14]. It provided a very uniform energy response and an accurate energy resolution of $2.0 \%$ for $100 \mathrm{GeV}$ electrons. The tungsten nose, which was used at LEP I to define the inner edge of the calorimeter, was removed between data taking in 1995 and 1996. After 1995 the definition of the geometrical acceptance was entirely based on the reconstructed radii of the electron and positron showers.

The trigger for Bhabha events was prescaled by a factor 3 to 4 to reduce the overall trigger rate. A comparison between the measured luminosity and the scalers of the trigger shows that the prescaling had no effect on the luminosity measurement [13]. Furthermore a prescaled single arm trigger was used to monitor possible trigger inefficiencies, which were found to be smaller than $2 \times 10^{-4}$.

In the selection of the Bhabha events, only the most energetic electromagnetic cluster in each arm of the STIC was used. To remove the background due to off-momentum particles, the following cuts were applied:

- in each arm, the energy of the cluster was required to be larger than $65 \%$ of the beam energy;

- the acoplanarity ${ }^{2}$ between the two clusters was required to be less than $20^{\circ}$.

A special trigger, requiring a coincidence between the signal from one arm of the STIC and a delayed signal from the other, measured the residual background due to off-momentum particles. The measurement showed that it was smaller than $10^{-4}$ of the accepted events.

In order to minimize the sensitivity of the accepted cross-section to the transverse position of the interaction point, an asymmetric acceptance was defined, with a narrow side and a wide side. The following cuts were applied to define the geometrical acceptance:

\footnotetext{
${ }^{2}$ In general the acoplanarity is defined as the complement of the angle, in the plane transverse to the beam, between two tracks. In this case the "tracks" are lines joining the two clusters to the interaction point.
} 


\begin{tabular}{|c||c|c|c|c|c|c|}
\hline Year & 1995 & 1996 & 1997 & 1998 & 1999 & 2000 \\
\hline \hline Number of Bhabha events $\left(\times 10^{3}\right)$ & 156 & 144 & 384 & 1195 & 856 & 757 \\
\hline
\end{tabular}

Table 2: Number of Bhabha events at LEP II.

- the radial position of the reconstructed shower was required to be between 10 and $25 \mathrm{~cm}$ on the narrow side;

- the radial position of the reconstructed shower was required to be between 9.1 and $28 \mathrm{~cm}$ on the wide side.

The cuts were chosen at the borders between rings, where the best spatial resolution is achieved. The narrow side was alternated between the forward and the backward hemispheres in successive events, in order to minimize the sensitivity to the longitudinal position of the interaction point.

The calculation of the accepted cross-section was based on the event generator BHLUMI 4.04 [15], which includes the full $\mathcal{O}\left(\alpha^{2}\right)$ calculation. The generated events were passed through a detailed simulation of the detector, and analysed in the same way as the real data. The total accepted cross-section was estimated to be $10.712 \pm 0.010 \mathrm{nb}$, at a centre-of-mass energy of $200 \mathrm{GeV}$. The contribution of the process $e^{+} e^{-} \rightarrow \gamma \gamma$ in the selected sample of Bhabha events was calculated to be $0.06 \%$. The theoretical uncertainty on the estimation of the cross-section is estimated to be $\pm 0.12 \%$ [16]. There is a factor of two improvement on the error quoted in [3,4]; in the analysis of the results and interpretations made in the paper these smaller errors have been applied to all data, including those data sets which have not been reanalysed in full.

The number of selected Bhabhas collected at LEP II energies during the different years of data taking are reported in Table 2 .

The experimental systematics in the luminosity measurement were dominated by the uncertainty in the radial cut which defines the inner border of the acceptance. The bias of the radius reconstruction was carefully studied by comparing the STIC measurement with the independent measurement from the two planes of the Silicon Shower Maximum Detectors, embedded inside the STIC. The difference between the two measurements was monitored throughout data taking and the bias was evaluated to be $250 \mu \mathrm{m}$, corresponding to a systematic uncertainty of $\pm 0.5 \%$. The other sources of uncertainties in the event selection were estimated to contribute $\pm 0.04 \%$ to the systematics. The luminosity had to be corrected for the average displacement of the interaction point from its nominal position, as measured by the DELPHI tracking system. The residual systematic uncertainty, connected with the fill-to-fill fluctuations, was evaluated to be $\pm 0.03 \%$. The uncertainty in the beam energy at LEP II propagated into a systematic uncertainty of $\pm 0.04 \%$. A detailed list of the contributions to the systematic uncertainty is given in Table 3 . The overall systematic uncertainty is evaluated to be $\pm 0.5 \%$ and it is common to all LEP II data taking, except for 1995. For 1995, the presence of the tungsten mask made the luminosity determination more accurate, giving an experimental systematic uncertainty of $\pm 0.09 \%$ on the luminosity determination, for data taken during that year. 


\begin{tabular}{|l|c|}
\hline Source & $\Delta \mathcal{L} / \mathcal{L}(\%)$ \\
\hline \hline Bias on inner edge of the acceptance & 0.5 \\
Beam energy & 0.04 \\
Position of the IP & 0.03 \\
Event selection & 0.04 \\
Background subtraction & 0.01 \\
Trigger inefficiency & 0.02 \\
\hline \hline Total experimental & 0.5 \\
\hline \hline Total theoretical & 0.12 \\
\hline
\end{tabular}

Table 3: Contributions to the systematic uncertainty of the luminosity measurement at LEP II energies from 1996 onwards. 

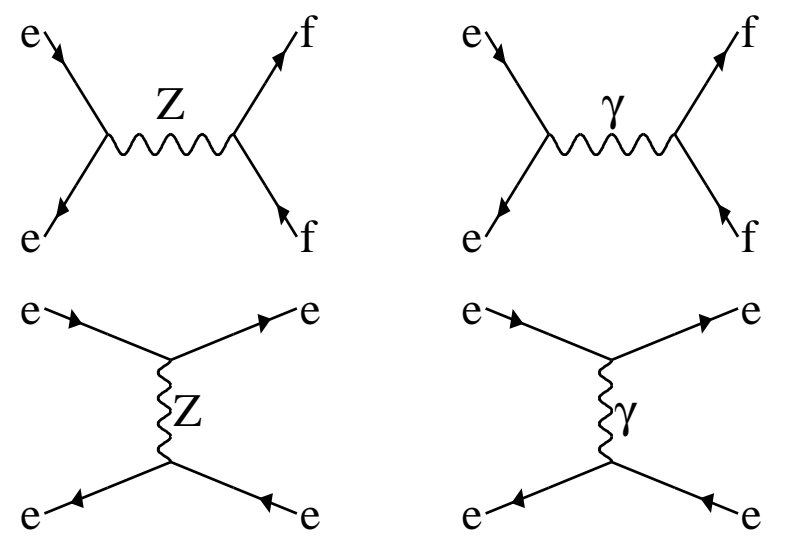

Figure 1: The Standard Model s-channel processes $\mathrm{e}^{+} \mathrm{e}^{-} \rightarrow \mathrm{f} \overline{\mathrm{f}}$ (upper) and t-channel processes in $\mathrm{e}^{+} \mathrm{e}^{-} \rightarrow \mathrm{e}^{+} \mathrm{e}^{-}$(lower).

\section{Analysis of $\mathrm{f} \overline{\mathrm{f}}$ final states}

The process $\mathrm{e}^{+} \mathrm{e}^{-} \rightarrow \mathrm{f} \overline{\mathrm{f}}$ at LEP II is very similar to that at LEP I. In the Standard Model the $s$-channel processes $\mathrm{e}^{+} \mathrm{e}^{-} \rightarrow \mu^{+} \mu^{-}, \mathrm{e}^{+} \mathrm{e}^{-} \rightarrow \tau^{+} \tau^{-}$and $\mathrm{e}^{+} \mathrm{e}^{-} \rightarrow \mathrm{q} \overline{\mathrm{q}}$ are described by the exchange of virtual photons and $\mathrm{Z}$ bosons, shown as Feynman diagrams in Figure 1. At LEP I the process is dominated by the formation and decay of the Z. At LEP II, the exchange of the photon and the interference between the $\mathrm{Z}$ and the photon (which is highly suppressed at the $\mathrm{Z}$ pole due to the phases of the $\mathrm{Z}$ and photon exchange amplitudes) are more important. Moreover, the predicted Born level cross-sections for the $s$-channel process are some $2-3$ orders of magnitude smaller than at the $\mathrm{Z}$ resonance, and the forward-backward asymmetries are larger.

At the peak of the $\mathrm{Z}$ resonance $\mathrm{QED}$ radiative corrections are significant, leading to $\mathrm{a} \sim 30 \%$ reduction of the cross-section relative to the Born level prediction. At LEP II energies, QED radiative corrections are again significant, but here they lead to an increase of a factor of $\mathcal{O}(2-3)$ in the total cross-section above the Born level predictions. This is predominantly through the process of radiative return, in which a photon is radiated from the incoming electron or positron, reducing the centre-of-mass energy of the hard scattering from the full centre-of-mass energy, $\sqrt{s}$, to close to the $\mathrm{Z}$ resonance. The photon is typically emitted along the direction of the incoming beams, and usually goes undetected down the LEP beampipe. The final-state fermion pairs in radiative return events are, therefore, usually acollinear, though they are typically produced back-to-back in the plane transverse to the incoming beams. An important step of the analysis for each channel is to determine the reduced centre-of-mass energy of the collisions, $\sqrt{s^{\prime}}$. This is used to separate events with high collision energies from radiative return events. Measured cross-sections and forward-backward asymmetries are quoted for non-radiative samples, for which $\sqrt{s^{\prime}} \sim \sqrt{s}$ and for inclusive samples of events, which also include radiative return events. The algorithms employed to determine $\sqrt{s^{\prime}}$ are different in each channel.

The process $\mathrm{e}^{+} \mathrm{e}^{-} \rightarrow \mathrm{e}^{+} \mathrm{e}^{-}$is dominated by the $t$-channel exchange of virtual photons. The $t$-channel processes are shown in Figure 1. At the $\mathrm{Z}$ resonance the cross-sections are sensitive to $s$-channel $\mathrm{Z}$ exchange. Therefore, at LEP I it was reasonable to subtract off the predicted $t$-channel contributions to measured quantities for $\mathrm{e}^{+} \mathrm{e}^{-} \rightarrow \mathrm{e}^{+} \mathrm{e}^{-}$collisions in 
order to extract Z parameters, such as the Z mass and coupling to electrons . Above the Z resonance, this subtraction is less appropriate. Physics beyond the Standard Model may manifest itself through $t$-channel processes. No subtraction of the $t$-channel contributions to the measured cross-sections, asymmetries or differential cross-sections has been made. A cut on the acollinearity of the electron-positron pair in the final state is used to separate events with high invariant masses from radiative return events.

In addition to falling cross-sections for the signal $\mathrm{e}^{+} \mathrm{e}^{-} \rightarrow \mathrm{f} \overline{\mathrm{f}}$ processes compared to LEP I, the backgrounds from other processes increase at LEP II. The cross-section for two-photon collision processes increases as log $s$ and new channels such as $\mathrm{e}^{+} \mathrm{e}^{-} \rightarrow \mathrm{W}^{+} \mathrm{W}^{-}$ and $\mathrm{e}^{+} \mathrm{e}^{-} \rightarrow \mathrm{ZZ}$ open at $\sqrt{s} \sim 2 \mathrm{M}_{\mathrm{W}}$ and $\sqrt{s} \sim 2 \mathrm{M}_{\mathrm{Z}}$ respectively.

With some small modifications to take into account the new radiative return topologies and the smaller signal over background ratio, the selection of $\mathrm{e}^{+} \mathrm{e}^{-} \rightarrow \mathrm{f} \overline{\mathrm{f}}$ events at LEP II is very similar to that at LEP I. Classification into $\mathrm{e}^{+} \mathrm{e}^{-}, \mu^{+} \mu^{-}, \tau^{+} \tau^{-}$and $\mathrm{q} \overline{\mathrm{q}}$ final states is based on the the multiplicity of final state particles, the responses of the electromagnetic and hadronic calorimeters and the muon chambers, and the momenta of charged particles measured in the tracking system.

\section{Event simulation}

To determine selection efficiencies and backgrounds for each analysis, events were simulated using a variety of event generators and the DELPHI detector simulation [11] and passed through the full data analysis chain. To allow studies of the data taken after September $1^{\text {st }} 2000$, samples of events were simulated dropping information from the broken sector of the TPC.

The $s$-channel $\mathrm{e}^{+} \mathrm{e}^{-} \rightarrow \mathrm{f} \bar{f}$ processes were simulated with KK 4.14 [17], while events in the $\mathrm{e}^{+} \mathrm{e}^{-} \rightarrow \mathrm{e}^{+} \mathrm{e}^{-}$channel were simulated with BHWIDE 1.01 [18]. The fragmentation of $q \bar{q}$ events into hadrons was simulated using PYTHIA 6.156 [19,20], with parameters tuned to DELPHI data [21]. Spin effects in $\tau$-lepton decays were handled by TAUOLA 2.6 [22] using the helicity approximation. There were typically 1 million simulated events per energy for the $\mathrm{e}^{+} \mathrm{e}^{-} \rightarrow \mathrm{e}^{+} \mathrm{e}^{-}$and $\mathrm{e}^{+} \mathrm{e}^{-} \rightarrow \mathrm{q} \overline{\mathrm{q}}$ channels, with 100,000 simulated events for the $\mathrm{e}^{+} \mathrm{e}^{-} \rightarrow \mu^{+} \mu^{-}$channel and 200,000 simulated events for the $\mathrm{e}^{+} \mathrm{e}^{-} \rightarrow \tau^{+} \tau^{-}$channel at each energy.

Four-fermion background events, including high-mass two-photon collision events, were simulated with the generator WPHACT 2.0 [23]. The generated events were divided into 3 samples [24]; the first dominated by charged current processes, $\mathrm{e}^{+} \mathrm{e}^{-} \rightarrow \mathrm{W}^{+} \mathrm{W}^{-}$; the second dominated by neutral current processes with topologies similar to $\mathrm{e}^{+} \mathrm{e}^{-} \rightarrow \mathrm{ZZ}$ events; and the third sample being neutral current samples dominated by multi-peripheral processes, $\mathrm{e}^{+} \mathrm{e}^{-} \rightarrow \mathrm{e}^{+} \mathrm{e}^{-} \mathrm{f} \overline{\mathrm{f}}$. Low mass two-photon collisions were simulated with BDKRC [25] for leptonic final states and with PYTHIA for hadronic final states. Since the samples of 4-fermion events were generated with certain kinematic cuts, the background subtraction involved an extrapolation to estimate the backgrounds coming from events which were not simulated. Theoretical uncertainties amount to $\pm 5 \%$ on the $\mathrm{e}^{+} \mathrm{e}^{-} \rightarrow \mathrm{e}^{+} \mathrm{e}^{-\mathrm{ff}}$ samples, $\pm 0.5 \%$ on the $\mathrm{e}^{+} \mathrm{e}^{-} \rightarrow \mathrm{W}^{+} \mathrm{W}^{-}$and $\pm 2 \%$ on the $\mathrm{e}^{+} \mathrm{e}^{-} \rightarrow \mathrm{ZZ}$ samples. Where possible the real data were used to control the simulation predictions for the backgrounds in the selected samples.

\section{Theoretical predictions and signal definition}

The measurements reported in this paper are compared to theoretical predictions, from the Standard Model and from models which include physics beyond the Standard Model. 
Throughout this paper Standard Model predictions are taken from the semi-analytical QED corrected, Improved Born Approximation computations of ZFITTER [26] for $\mathrm{e}^{+} \mathrm{e}^{-} \rightarrow \mu^{+} \mu^{-}, \mathrm{e}^{+} \mathrm{e}^{-} \rightarrow \tau^{+} \tau^{-}$and $\mathrm{e}^{+} \mathrm{e}^{-} \rightarrow \mathrm{q} \overline{\mathrm{q}}$, and from BHWIDE [18] for $\mathrm{e}^{+} \mathrm{e}^{-} \rightarrow \mathrm{e}^{+} \mathrm{e}^{-}$. ZFITTER version $6.36^{3}$ was used with the following central values for input parameters

$$
\begin{aligned}
\mathrm{M}_{\mathrm{Z}} & =91.1875 \mathrm{GeV} / c^{2} \\
\mathrm{~m}_{\mathrm{t}} & =175.0 \mathrm{GeV} / c^{2} \\
\mathrm{M}_{\mathrm{H}} & =150.0 \mathrm{GeV} / c^{2} \\
\alpha_{s}\left(\mathrm{M}_{\mathrm{Z}}\right) & =0.118 \\
\Delta \alpha_{\text {had }}^{(5)} & =0.02761
\end{aligned}
$$

where $\Delta \alpha_{\text {had }}^{(5)}$ is the contribution to the running of the electromagnetic coupling constant, $\alpha$, due to contributions from hadronic loops containing 5 quark flavours. To make the comparison it was necessary to match the signal definitions in the data, simulation and semi-analytical computations. There were several choices to be made: definition of $\sqrt{s^{\prime}}$; subtraction or inclusion of QED corrections from the interference between Initial State Radiation (ISR) and Final Sate Radiation (FSR); inclusion of $\mathrm{f} \overline{\mathrm{f}}$ events with additional radiated pairs of fermions; angular acceptance and $\sqrt{s^{\prime}}$ acceptance. The signal definition adopted here was chosen to make analysis of the non-radiative events as straightforward as possible:

- for $\mathrm{e}^{+} \mathrm{e}^{-} \rightarrow \mu^{+} \mu^{-}$and $\mathrm{e}^{+} \mathrm{e}^{-} \rightarrow \tau^{+} \tau^{-}, \sqrt{s^{\prime}}$ is taken to be the invariant mass of the fermion pair, $\mathrm{M}_{\mathrm{f} \overline{\mathrm{f}}}$. For $\mathrm{e}^{+} \mathrm{e}^{-} \rightarrow \mathrm{q} \overline{\mathrm{q}}, \sqrt{s^{\prime}}$ is taken to be the invariant mass of the fermion pair with any FSR included - for $s$-channel processes this corresponds to computing the invariant mass, $Q$, of the virtual propagator;

- QED corrections from the interference between ISR and FSR are included for $\mathrm{e}^{+} \mathrm{e}^{-} \rightarrow \mu^{+} \mu^{-}$and $\mathrm{e}^{+} \mathrm{e}^{-} \rightarrow \tau^{+} \tau^{-}$. These corrections are included in the simulated events. For $\mathrm{e}^{+} \mathrm{e}^{-} \rightarrow \mathrm{q} \overline{\mathrm{q}}$ these corrections are not included in the simulated events, furthermore, the definition $\sqrt{s^{\prime}}=Q$ is ambiguous in the presence of interference between ISR and FSR. Therefore, for the $\mathrm{e}^{+} \mathrm{e}^{-} \rightarrow \mathrm{q} \overline{\mathrm{q}}$ channel, the signal is defined as having no ISR-FSR interference. Corrections are applied to the data to remove the effects of the interference;

- events with additional radiated fermion pairs are subtracted as part of the 4-fermion background. The bulk of the pairs come from internal conversion of ISR photons into $\mathrm{e}^{+} \mathrm{e}^{-}$pairs, which are lost in the beampipe, and which are topologically equivalent to radiative return events. However, for the non-radiative samples this background is small compared to the signal;

- the total cross-sections and forward-backward asymmetries are quoted in the full $4 \pi$ acceptance for $\mathrm{e}^{+} \mathrm{e}^{-} \rightarrow \mu^{+} \mu^{-}, \mathrm{e}^{+} \mathrm{e}^{-} \rightarrow \tau^{+} \tau^{-}$and $\mathrm{e}^{+} \mathrm{e}^{-} \rightarrow \mathrm{q} \overline{\mathrm{q}}$. For $\mathrm{e}^{+} \mathrm{e}^{-} \rightarrow \mu^{+} \mu^{-}$and $\mathrm{e}^{+} \mathrm{e}^{-} \rightarrow \tau^{+} \tau^{-}$this involves an extrapolation from the fiducial volume of the detector using generated events. For the electrons the measurements are made within the acceptance $44^{\circ}<\theta<136^{\circ}$ - which corresponds to the acceptance of the HPC. Measurements in the FEMC region, $12^{\circ}<\theta<35^{\circ}$ and $145^{\circ}<\theta<168^{\circ}$, are not reported here, since they are subject to greater experimental uncertainties and the

\footnotetext{
${ }^{3}$ The following ZFITTER flags were used: AFBC: 1 SCAL: 0 SCRE: 0 AMT4: 4 BORN: 0 BOXD: 2 CONV: 2 FOT2: 3 GAMS: 1 DIAG: 1 BARB: 2 PART: 0 POWR: 1 PRNT: 0 ALEM: 2 QCDC: 3 VPOL: 1 WEAK: 1 FTJR: 1 EXPR: 0 EXPF: 0 HIGS: 0 AFMT: 1 CZAK: 0 PREC:10 HIG2: 0 ALE2: 3 GFER: 2 ISPP: 2 FSRS: 1 MISC: 0 MISD: 1 IPFC: 5 IPSC: 0 IPTO:-1 FBHO: 0 FSPP: 0 FUNA: 0 ASCR: 1 SFSR: 1 ENUE: 1 TUPV: 1 . For $\mathrm{e}^{+} \mathrm{e}^{-} \rightarrow$ q $\overline{\mathrm{q}}$ FINR: 0 INTF: 0, while for $\mathrm{e}^{+} \mathrm{e}^{-} \rightarrow \mu^{+} \mu^{-}$and $\mathrm{e}^{+} \mathrm{e}^{-} \rightarrow \tau^{+} \tau^{-}$FINR: 1 INTF: 2 For non-radiative samples FSRS: 0, while for inclusive samples FSRS: 1.
} 
cross-sections are dominated by the $t$-channel process. The differential cross-sections for $\mathrm{e}^{+} \mathrm{e}^{-} \rightarrow \mu^{+} \mu^{-}$and $\mathrm{e}^{+} \mathrm{e}^{-} \rightarrow \tau^{+} \tau^{-}$are quoted within the fiducial volume of the detector, with a cut at $|\cos \theta|=0.97$ for $\mathrm{e}^{+} \mathrm{e}^{-} \rightarrow \mu^{+} \mu^{-}$and 0.96 for $\tau^{+} \tau^{-}$final states;

- for the inclusive samples the $\sqrt{s^{\prime}}$ acceptances for $\mu^{+} \mu^{-}, \tau^{+} \tau^{-}$final states are $\sqrt{s^{\prime}}>75 \mathrm{GeV}$ and $\sqrt{s^{\prime}}>0.10 \sqrt{s}$ for $\mathrm{q} \overline{\mathrm{q}}$ final states. For the non-radiative samples $\sqrt{s^{\prime}} / \sqrt{s}>0.85$ for all these processes. For $\mathrm{e}^{+} \mathrm{e}^{-}$final states the cut on $\sqrt{s^{\prime}}$ is replaced by a cut on the acollinearity, $\theta_{\text {acol }}<20^{\circ}$; both $s$ and $t$ channel processes leading to $\mathrm{e}^{+} \mathrm{e}^{-}$final states are considered as signal.

In the following sections the analyses of the different final states are discussed and results of the measurements are presented. In all cases the latest theoretical predictions have been used, updating values given in previous publications $[3,4]$.

\section{Experimental uncertainties}

Although a large number of possible biases and sources of uncertainty were investigated for all final states, only those sources of bias and uncertainty which lead to significant systematic errors for each particular analysis are described in the sections below. Sources of bias and uncertainty which are negligible for a particular channel are not described.

For measurements of the differential cross-sections for the $\mathrm{e}^{+} \mathrm{e}^{-} \rightarrow \mu^{+} \mu^{-}$and $\mathrm{e}^{+} \mathrm{e}^{-} \rightarrow \tau^{+} \tau^{-}$channels, some of the bins used for the analysis contain only a small number of observed events. For these measurements statistical errors were computed both from the square root of the number of observed events and of the number of events expected from the Standard Model. The second error provides a suitable weight which can be used to combine measurements from different energies and can also be used when combining data from different LEP experiments or fitting small deviations from the Standard Model to the measurements. In all other cases the statistical errors were computed solely from the square root of the number of observed events.

\section{Analyses of the various final states}

\section{$5.1 \mathrm{e}^{+} \mathrm{e}^{-}$final states}

An analysis of $\mathrm{e}^{+} \mathrm{e}^{-}$final states at $\mathrm{e}^{+} \mathrm{e}^{-}$collision energies of $189 \mathrm{GeV}$ and above is presented. This updates the analysis of data taken at $189 \mathrm{GeV}$ [4]. Compared to the previous analysis, an explicit correction is applied for charge misidentification in the measurement of the forward-backward asymmetry. New results are presented for differential cross-sections. Data taken at collision energies below $189 \mathrm{GeV}$ [3, 4], have not been reanalysed.

\subsubsection{Analysis}

\section{Run Selection}

Runs were selected requiring that the Vertex Detector (VD), the TPC and the electromagnetic calorimeters in the barrel of DELPHI (HPC) were operative. For the 2000 data fraction with a TPC sector dead, the requirement for the TPC was restricted to the remaining sectors of the detector. 


\section{Event selection}

Events were selected with two almost-independent sets of experimental cuts, chosen in such a way as to minimize the correlations between the two sets $[1,2]$. Only the barrel region of DELPHI was used for this analysis. In each selection, both the electron and the positron were required to be within the range $44^{\circ}<\theta<136^{\circ}$ and the acollinearity was required to be smaller than $20^{\circ}$. A cut in polar angle at $90 \pm 2^{\circ}$ was applied to remove the region with neither TPC nor HPC coverage.

The first set of cuts (selection A) is based on requirements of calorimetric-energy clusters associated to hits in the Vertex Detector. In particular events were selected if they had:

- at least two electromagnetic clusters in the HPC one with energy above $75 \%$ of the beam energy and another above $55 \%$ and with an acollinearity angle between the clusters less than $20^{\circ}$;

- at least two track segments in opposite hemispheres seen by the VD and no more than four in total; events with a 2-versus-2 topology were excluded;

- no energy in the last two layers of the hadron calorimeter (HCAL) in correspondence with electromagnetic clusters detected at large distance $\left(> \pm 1^{\circ}\right.$ in $\left.\phi\right)$ from the HPC sector boundaries.

The second selection (selection B) is based on the charged-particle momentum as measured by the tracking system. In particular events were selected if they had:

- at least 2 charged-particle tracks, of momentum greater than $1.5 \mathrm{GeV} / c$ and distance of closest approach to the nominal vertex position less than $5 \mathrm{~cm}$, seen by the DELPHI tracking system outside the VD, with acollinearity less than $20^{\circ}$ and no more than four tracks in total; the 2-versus-2 track topology was excluded;

- the quadratic sum $\left(p_{\text {rad }}\right)$ of the two momenta of the highest-momentum charged particle in each hemisphere greater than $0.99 \sqrt{s} / 2$;

- no energy observed in the last three layers of HCAL associated to the impact points of the two highest-momentum charged particles;

- the OD hit pattern associated to the impact points of the tracks compatible with the pattern of a particle showering in or before the OD, or giving back-scattering from the HPC calorimeter;

- no tracks identified as a muon.

\section{Estimation of the selection efficiency and background}

Considering the selections $\mathrm{A}$ and $\mathrm{B}$ as independent, the efficiency of each of them could be easily computed by a comparison of the number of events selected by each one separately or by both simultaneously. This is much easier at LEP II energies, given the $t$-channel dominance, compared to the analysis at the $\mathrm{Z}$ resonance because of the much smaller background ( $\tau$ events estimated from simulation) as shown in Figure 2(left).

The efficiency of the two selections at the different energy points is given in Table 4 . In both selections the measured efficiencies did not include the loss due to the exclusion of the polar angle region around $90^{\circ}$. The total number of events selected by selection $\mathrm{A}$ at the different centre-of-mass energies is shown in Table 4.

The simulated $\mathrm{e}^{+} \mathrm{e}^{-}$events were used to estimate and remove the bias caused by the correlation between the two selections due to the detector structure or to the kinematics of the events. The bias on the efficiency was found to be $(0.4 \pm 0.7) \%$. However in 
DELPHI

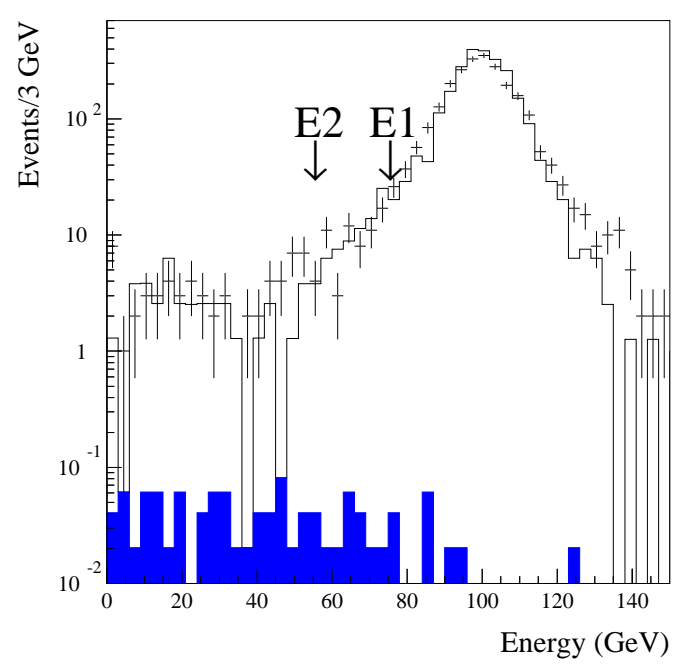

DELPHI

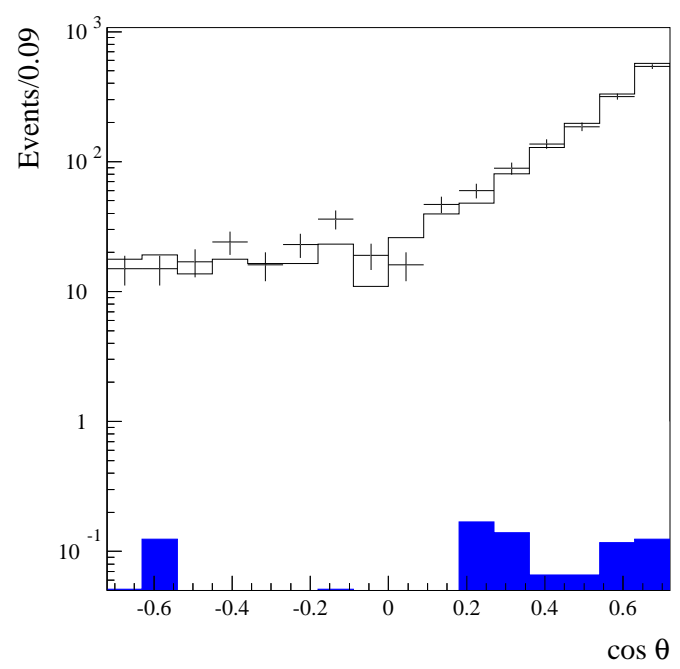

Figure 2: The energy of the two most energetic clusters (left) and the uncorrected $\cos \theta$ distribution (right). Both distributions correspond to data taken at $\sqrt{s} \sim 200$ GeV and the left plot does not include the energy cuts (indicated by the arrows) applied in selection A. The histogram corresponds to the simulated events and the dark area shows the $\tau$ background. 


\begin{tabular}{|c|c|c|c|c|c|c|c|}
\hline \multicolumn{8}{|c|}{$\mathrm{e}^{+} \mathrm{e}^{-} \rightarrow \mathrm{e}^{+} \mathrm{e}^{-}$} \\
\hline & \multicolumn{7}{|c|}{ Energy point $(\mathrm{GeV})$} \\
\hline & 189 & 192 & 196 & 200 & 202 & 205 & 207 \\
\hline Energy $(\mathrm{GeV})$ & 188.63 & 192.17 & 196.10 & 200.12 & 202.07 & 204.88 & 206.59 \\
\hline Lumi $\left(\mathrm{pb}^{-1}\right)$ & 155.11 & 25.12 & 76.16 & 83.07 & 40.05 & 88.55 & 128.39 \\
\hline No. Events (A) & 3179 & 518 & 1568 & 1554 & 778 & 1500 & 2126 \\
\hline Efficiency (A) (\%) & 91.9 & 92.3 & 93.7 & 92.8 & 93.6 & 92.6 & 92.6 \\
\hline Efficiency (B) (\%) & 77.4 & 76.6 & 76.4 & 76.7 & 75.1 & 72.6 & 72.6 \\
\hline Background (\%) & 0.2 & 0.2 & 0.2 & 0.2 & 0.2 & 0.2 & 0.2 \\
\hline
\end{tabular}

Table 4: Details of LEP II analysis for $\mathrm{e}^{+} \mathrm{e}^{-} \rightarrow \mathrm{e}^{+} \mathrm{e}^{-}$channel. The table shows the actual centre-of-mass energy and luminosity analysed at each energy point, the number of events selected by selection $A$ and the efficiencies of selecting $\mathrm{e}^{+} \mathrm{e}^{-} \rightarrow \mathrm{e}^{+} \mathrm{e}^{-}$events with selections $A$ and $B$ and the background selected with selection $A$.

the 2000 data new algorithms were used for track reconstruction based on VD hits. An increased correlation between the two selections was expected. In order to measure it, 1999 data reconstructed with the new and the old algorithm were compared. This gave a correction factor of $1.016 \pm 0.003$ to be applied to the cross-section measurements of the 2000 data.

\subsubsection{Results}

\section{Cross-sections}

The cross-section was obtained as:

$$
\sigma_{e e}=\frac{N_{A}}{\mathcal{L} \epsilon_{A}} \cdot c_{f}+\sigma^{90},
$$

where $N_{A}$ is the number of events selected by selection A and $\epsilon_{A}$ is the efficiency of this selection, $\mathcal{L}$ is the luminosity, $c_{f}$ is a correction factor including the bias in the evaluation of $\epsilon_{A}$ due to the correlation between the two selection methods and also the background subtraction, and $\sigma^{90}$ is the correction for the central region $(90 \pm 2)^{\circ}$. The value of $\sigma^{90}$ was computed by TOPAZ0 [27] and ALIBABA [28] and its value ranges from $0.43 \pm 0.04 \mathrm{pb}$ to $0.36 \pm 0.04 \mathrm{pb}$.

The statistical error on the cross-section includes the statistical uncertainty on the determination of the efficiency, taking into account the statistical uncertainties on correlated and uncorrelated subsamples from selections A and B.

All the values of the measured cross-sections are given in Table 5 and shown in Figure 10 .

\section{Forward-backward asymmetries}

In order to measure the forward-backward asymmetry the charge of the event was defined as positive when the positron was in the forward hemisphere with respect to the incoming positron direction, and negative in the opposite case. In the $\mathrm{e}^{+} \mathrm{e}^{-} \rightarrow \mathrm{e}^{+} \mathrm{e}^{-}$ events, in addition to the canonical charge definition from reconstructed tracks, it is 


\begin{tabular}{|c|c|c|c|c|c|}
\hline \multicolumn{6}{|c|}{$\mathrm{e}^{+} \mathrm{e}^{-} \rightarrow \mathrm{e}^{+} \mathrm{e}^{-}$} \\
\hline \multirow{2}{*}{$\begin{array}{c}\sqrt{s} \\
(\mathrm{GeV})\end{array}$} & \multicolumn{2}{|c|}{$\theta_{\text {acol }}<20^{\circ}$} & \multirow{2}{*}{$\begin{array}{c}\sqrt{s} \\
(\mathrm{GeV}) \\
\end{array}$} & \multicolumn{2}{|c|}{$\theta_{\text {acol }}<20^{\circ}$} \\
\hline & $\sigma_{e e}(\mathrm{pb})$ & $\mathrm{A}_{\mathrm{FB}}^{\mathrm{e}}$ & & $\sigma_{e e}(\mathrm{pb})$ & $\mathrm{A}_{\mathrm{FB}}^{\mathrm{e}}$ \\
\hline 130 & $\begin{array}{c}42.00 \pm 4.00 \pm 0.78 \\
(48.70)\end{array}$ & $\begin{array}{c}0.810 \pm 0.060 \pm 0.003 \\
(0.810)\end{array}$ & 192 & $\begin{array}{c}22.71 \pm 0.98 \pm 0.24 \\
(22.13)\end{array}$ & $\begin{array}{c}0.831 \pm 0.024 \pm 0.003 \\
(0.820)\end{array}$ \\
\hline 136 & $\begin{array}{c}47.10 \pm 4.20 \pm 0.73 \\
(44.60)\end{array}$ & $\begin{array}{c}0.890 \pm 0.040 \pm 0.003 \\
(0.810)\end{array}$ & 196 & $\begin{array}{c}22.33 \pm 0.55 \pm 0.23 \\
(21.24)\end{array}$ & $\begin{array}{c}0.823 \pm 0.014 \pm 0.003 \\
(0.821)\end{array}$ \\
\hline 161 & $\begin{array}{c}27.10 \pm 1.80 \pm 0.43 \\
(31.90)\end{array}$ & $\begin{array}{c}0.820 \pm 0.040 \pm 0.003 \\
(0.830)\end{array}$ & 200 & $\begin{array}{c}20.52 \pm 0.51 \pm 0.21 \\
(20.36)\end{array}$ & $\begin{array}{c}0.788 \pm 0.016 \pm 0.003 \\
(0.823)\end{array}$ \\
\hline 172 & $\begin{array}{c}30.30 \pm 1.90 \pm 0.45 \\
(28.00)\end{array}$ & $\begin{array}{c}0.810 \pm 0.040 \pm 0.003 \\
(0.830)\end{array}$ & 202 & $\begin{array}{c}21.11 \pm 0.74 \pm 0.22 \\
(19.97)\end{array}$ & $\begin{array}{c}0.831 \pm 0.020 \pm 0.003 \\
(0.822)\end{array}$ \\
\hline 183 & $\begin{array}{c}25.63 \pm 0.76 \pm 0.26 \\
(24.54)\end{array}$ & $\begin{array}{c}0.814 \pm 0.017 \pm 0.003 \\
(0.817)\end{array}$ & 205 & $\begin{array}{c}18.94 \pm 0.48 \pm 0.21 \\
(19.33)\end{array}$ & $\begin{array}{c}0.797 \pm 0.016 \pm 0.004 \\
(0.820)\end{array}$ \\
\hline 189 & $\begin{array}{c}22.73 \pm 0.40 \pm 0.23 \\
(22.93)\end{array}$ & $\begin{array}{c}0.804 \pm 0.010 \pm 0.003 \\
(0.820)\end{array}$ & 207 & $\begin{array}{c}18.52 \pm 0.39 \pm 0.20 \\
(19.07)\end{array}$ & $\begin{array}{c}0.820 \pm 0.012 \pm 0.004 \\
(0.822)\end{array}$ \\
\hline
\end{tabular}

Table 5: Measured cross-sections and forward-backward asymmetries for non-radiative $\mathrm{e}^{+} \mathrm{e}^{-} \rightarrow \mathrm{e}^{+} \mathrm{e}^{-}$events. The statistical error is followed by the total systematic error. In parentheses the expected values as computed by BHWIDE, which have a precision of $\pm 2 \%$ on $\sigma_{e e}$ and \pm 0.02 on $\mathrm{A}_{\mathrm{FB}}^{\mathrm{e}}$, are given. Results are quoted for an acceptance of $44^{\circ}<\theta<136^{\circ}$. 
possible to correlate the charges of an event by looking at the effects of the bending due to the magnetic field on the impact position of HPC clusters, giving a high redundancy on the charge determination. The latter method was used to determine the charge of an event in cases where the reconstructed charges of the tracks were equal.

Given the high expected asymmetry the measurements have to be corrected for the residual wrong charge assignments. The asymmetry was corrected by a factor $1+2 \xi_{ \pm}$with $\xi_{ \pm}=(7.2 \pm 1.4) \cdot 10^{-3}$ corresponding to the charge misassignment which was determined from simulated events:

$$
A_{F B}^{0}=\frac{N_{+}-N_{-}}{N_{+}+N_{-}} \cdot\left(1+2 \xi_{ \pm}\right)
$$

where $N_{+}$and $N_{-}$are the number of events with positive and negative charge, respectively. In the previous publications this correction was not applied and an asymmetric error was given. The forward-backward asymmetry is corrected also for the missing central region:

$$
A_{F B}=A_{F B}^{0} \cdot \frac{\sigma_{e e}-\sigma^{90}}{\sigma_{e e}}+\frac{\sigma_{+}^{90}-\sigma_{-}^{90}}{\sigma_{e e}},
$$

where $\sigma_{+}^{90}$ and $\sigma_{-}^{90}$ are the computed cross-sections for the regions $\left[88^{\circ}, 90^{\circ}\right]$ and $\left[90^{\circ}, 92^{\circ}\right]$ respectively.

All the values of the measured forward-backward asymmetries are given in Table 5 and shown in Figure 11.

\section{Differential cross-sections}

As with the measurement of the forward-backward asymmetry, measurements of the differential cross-sections require the determination of the charge of the electron and positron, and a correction has to be applied for mismeasurements of the charge. A bin by bin correction was applied to the events detected in each polar angle bin $N_{\theta_{i}}$ which was largest in the very forward and backward bins due to the large asymmetry in the cross-sections:

$$
N_{\theta_{i}}^{c}=N_{\theta_{i}} \cdot\left(1+\xi_{ \pm}\right)-N_{\bar{\theta}_{i}} \cdot\left(\xi_{ \pm}\right),
$$

where $N_{\bar{\theta}_{i}}$ is the number of events detected in the opposite hemisphere. The bin $-0.72<$ $\cos \theta<-0.54$ has the largest correction which is about 25 to $30 \%$. The differential cross-section is obtained as

$$
\mathrm{d} \sigma / \mathrm{d} \cos \theta_{i}=\frac{N_{\theta_{i}}^{c}}{\mathcal{L} \epsilon_{\theta_{i}} \cdot \Delta \cos \theta},
$$

where $\epsilon_{\theta_{i}}$ is a bin correction factor dominated by the efficiency evaluation and $\Delta \cos \theta$ is the bin width. The $90^{\circ}$ region correction is taken into account by adding $\sigma_{+}^{90} / \Delta \cos \theta$ and $\sigma_{-}^{90} / \Delta \cos \theta$ to the corresponding bins. The uncorrected $\cos \theta$ distribution is shown in Figure 2(right). The differential cross-sections for the 1998, 1999 and 2000 data are given in Table 6.

\section{Systematic errors}

Apart from the luminosity, systematic uncertainties arise from the event selection correlation, acceptance definition and from the background subtraction. The largest uncertainty comes from the selection correlation $( \pm 0.7 \%)$ due to the statistics of simulated events. The simulated data for 2000 were produced exclusively with a new tracking algorithm giving large correlations (which were measured directly from the data) and 


\begin{tabular}{|c|c|c|c|c|c|}
\hline & & & \multicolumn{3}{|c|}{$\mathrm{e}^{+} \mathrm{e}^{-} \rightarrow \mathrm{e}^{+} \mathrm{e}^{-}$} \\
\hline \multicolumn{3}{|c|}{$\sqrt{s} \sim 189$} & \multicolumn{3}{|c|}{$\sqrt{s} \sim 192$} \\
\hline \multirow[b]{2}{*}{$\cos \theta$} & \multicolumn{2}{|c|}{$\mathrm{d} \sigma / \mathrm{d} \cos \theta(\mathrm{pb})$} & \multirow[b]{2}{*}{$\cos \theta$} & \multicolumn{2}{|c|}{$\mathrm{d} \sigma / \mathrm{d} \cos \theta(\mathrm{pb})$} \\
\hline & $\mathrm{SM}$ & Measurement & & $\mathrm{SM}$ & Measurement \\
\hline$[-0.72,-0.54]$ & 1.89 & $2.69 \pm 0.31 \pm 0.09$ & {$[-0.72,-0.54]$} & 1.81 & $2.03 \pm 0.73 \pm 0.09$ \\
\hline$[-0.54,-0.36]$ & 2.36 & $2.31 \pm 0.32 \pm 0.04$ & {$[-0.54,-0.36]$} & 2.29 & $1.87 \pm 0.80 \pm 0.04$ \\
\hline$[-0.36,-0.18]$ & 2.98 & $2.53 \pm 0.34 \pm 0.03$ & {$[-0.36,-0.18]$} & 2.93 & $2.40 \pm 0.87 \pm 0.03$ \\
\hline$[-0.18,0.00]$ & 4.23 & $4.73 \pm 0.36 \pm 0.23$ & {$[-0.18,0.00]$} & 4.05 & $5.36 \pm 1.00 \pm 0.23$ \\
\hline$[0.00,0.09]$ & 5.86 & $5.27 \pm 0.49 \pm 0.34$ & {$[0.00,0.09]$} & 5.64 & $4.05 \pm 1.40 \pm 0.34$ \\
\hline$[0.09,0.18]$ & 7.55 & $8.18 \pm 0.77 \pm 0.07$ & $0.09,0.18]$ & 7.44 & $11.39 \pm 2.18 \pm 0.08$ \\
\hline $0.18,0.27$ & 10.16 & $9.79 \pm 0.88 \pm 0.10$ & $0.18,0.27]$ & 9.83 & $9.74 \pm 2.20 \pm 0.10$ \\
\hline $0.27,0.36]$ & 14.44 & $12.58 \pm 1.06 \pm 0.14$ & $0.27,0.36]$ & 13.80 & $12.03 \pm 2.64 \pm 0.14$ \\
\hline $0.36,0.45$ & 21.15 & $21.48 \pm 1.31 \pm 0.21$ & $0.36,0.45]$ & 20.35 & $20.72 \pm 3.34 \pm 0.21$ \\
\hline $0.45,0.54]$ & 32.75 & $31.21 \pm 1.60 \pm 0.32$ & $0.45,0.54]$ & 31.32 & $29.61 \pm 3.88 \pm 0.32$ \\
\hline $0.54,0.63]$ & 52.28 & $52.76 \pm 2.04 \pm 0.51$ & $0.54,0.63]$ & 50.21 & $54.58 \pm 4.79 \pm 0.51$ \\
\hline $0.63,0.72]$ & 87.65 & $87.85 \pm 2.59 \pm 0.86$ & $0.63,0.72]$ & 85.17 & $87.93 \pm 6.28 \pm 0.87$ \\
\hline \multicolumn{3}{|c|}{$\sqrt{s} \sim 200$} & \multicolumn{3}{|c|}{$\sqrt{s} \sim 202$} \\
\hline & \multicolumn{2}{|c|}{$\mathrm{d} \sigma / \mathrm{d} \cos \theta(\mathrm{pb})$} & & \multicolumn{2}{|c|}{$\mathrm{d} \sigma / \mathrm{d} \cos \theta(\mathrm{pb})$} \\
\hline $\cos \theta$ & SM & Measurement & $\cos \theta$ & SM & Measurement \\
\hline$[-0.72,-0.54]$ & 1.57 & $1.73 \pm 0.38 \pm 0.08$ & {$[-0.72,-0.54]$} & 1.61 & $1.50 \pm 0.55 \pm 0.09$ \\
\hline$[-0.54,-0.36]$ & 2.07 & $2.81 \pm 0.40 \pm 0.03$ & {$[-0.54,-0.36]$} & 2.03 & $1.96 \pm 0.57 \pm 0.04$ \\
\hline$[-0.36,-0.18]$ & 2.66 & $2.86 \pm 0.45 \pm 0.03$ & {$[-0.36,-0.18]$} & 2.58 & $2.98 \pm 0.62 \pm 0.03$ \\
\hline$[-0.18,0.00]$ & 3.73 & $4.95 \pm 0.48 \pm 0.23$ & {$[-0.18,0.00]$} & 3.68 & $3.40 \pm 0.64 \pm 0.23$ \\
\hline$[0.00,0.09]$ & 5.23 & $5.23 \pm 0.73 \pm 0.34$ & {$[0.00,0.09]$} & 4.99 & $4.55 \pm 0.86 \pm 0.34$ \\
\hline$[0.09,0.18]$ & 6.72 & $6.26 \pm 0.95 \pm 0.07$ & $0.09,0.18]$ & 6.63 & $6.16 \pm 1.39 \pm 0.07$ \\
\hline $0.18,0.27$ & 9.02 & $9.14 \pm 1.17 \pm 0.09$ & $0.18,0.27]$ & 8.90 & $5.82 \pm 1.57 \pm 0.09$ \\
\hline $0.27,0.36$ & 12.85 & $13.16 \pm 1.37 \pm 0.13$ & $0.27,0.36]$ & 12.44 & $13.02 \pm 1.93 \pm 0.13$ \\
\hline $0.36,0.45]$ & 18.57 & $20.06 \pm 1.64 \pm 0.18$ & $0.36,0.45]$ & 18.44 & $18.85 \pm 2.40 \pm 0.19$ \\
\hline $0.45,0.54]$ & 29.03 & $26.43 \pm 2.03 \pm 0.29$ & $0.45,0.54]$ & 28.11 & $33.61 \pm 2.85 \pm 0.28$ \\
\hline $0.54,0.63]$ & 46.21 & $46.00 \pm 2.58 \pm 0.46$ & $0.54,0.63]$ & 45.70 & $48.97 \pm 3.74 \pm 0.46$ \\
\hline \multirow[t]{16}{*}{$0.63,0.72]$} & 78.54 & $77.96 \pm 3.34 \pm 0.78$ & $0.63,0.72]$ & 76.92 & $84.69 \pm 4.74 \pm 0.78$ \\
\hline & & & \multicolumn{3}{|c|}{$\sqrt{s} \sim 207$} \\
\hline & & & & \multicolumn{2}{|c|}{$\mathrm{d} \sigma / \mathrm{d} \cos \theta(\mathrm{pb})$} \\
\hline & & & $\cos \theta$ & $\mathrm{SM}$ & Measurement \\
\hline & & & {$[-0.72,-0.54]$} & 1.53 & 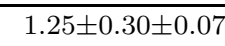 \\
\hline & & & {$[-0.54,-0.36]$} & 1.95 & $2.25 \pm 0.32 \pm 0.03$ \\
\hline & & & {$[-0.36,-0.18]$} & 2.55 & $2.11 \pm 0.35 \pm 0.03$ \\
\hline & & & {$[-0.18,0.00]$} & 3.41 & $3.85 \pm 0.36 \pm 0.23$ \\
\hline & & & {$[0.00,0.09]$} & 4.84 & $5.84 \pm 0.49 \pm 0.34$ \\
\hline & & & $0.09,0.18$ & 6.34 & $6.30 \pm 0.81 \pm 0.07$ \\
\hline & & & $0.18,0.27]$ & 8.30 & $7.59 \pm 0.89 \pm 0.09$ \\
\hline & & & $0.27,0.36]$ & 11.82 & $10.86 \pm 1.06 \pm 0.12$ \\
\hline & & & $0.36,0.45]$ & 17.70 & $16.84 \pm 1.29 \pm 0.19$ \\
\hline & & & $0.45,0.54]$ & 26.95 & $25.22 \pm 1.60 \pm 0.28$ \\
\hline & & & $0.54,0.63]$ & 43.65 & $42.40 \pm 2.03 \pm 0.46$ \\
\hline & & & $0.63,0.72]$ & 73.37 & $72.69 \pm 2.60 \pm 0.77$ \\
\hline
\end{tabular}

Table 6: Differential cross-sections for non-radiative $\mathrm{e}^{+} \mathrm{e}^{-} \rightarrow \mathrm{e}^{+} \mathrm{e}^{-}$events at centre-of-mass energies from $\sim 189$ to $207 \mathrm{GeV}$. The tables show the bins and the predicted Standard Model differential cross-sections and the measurements with statistical and experimental systematic errors. 


\begin{tabular}{|l|c||c|}
\hline \multicolumn{3}{|c|}{$\mathrm{e}^{+} \mathrm{e}^{-} \rightarrow \mathrm{e}^{+} \mathrm{e}^{-}$} \\
\hline \hline Source & $\begin{array}{c}\Delta \sigma / \sigma \\
\text { (non-rad.) }\end{array}$ & $\begin{array}{c}\Delta A_{F B} \\
\text { (non-rad.) }\end{array}$ \\
\hline \hline Efficiency & 76 & - \\
Acceptance & 48 & 20 \\
Charge misidentification & - & 20 \\
Backgrounds & 20 & 20 \\
Luminosity & 58 & - \\
TPC sector instability & - & 20 \\
\hline \hline Total uncertainty & 109 & 35 \\
\hline Correlated & 102 & 35 \\
Uncorrelated & 38 & - \\
\hline
\end{tabular}

Table 7: Systematic uncertainties on the measured cross-sections and forward-backward asymmetries for data taken at $\sqrt{s} \sim 207$ GeV. All numbers in units of $10^{-4}$. The total uncertainty does not include the error due to TPC sector instability which applies only to a part of the 2000 data. The correlated error component includes errors correlated between energies and channels and those correlated with other LEP experiments. 
therefore the statistical error cannot be reduced. The error on the cross-section due to the acceptance definition arises from the uncertainty on the absolute polar angle determination. For events having fully reconstructed charged particle tracks this uncertainty is very small $\left(<0.02^{\circ}\right)$, while for those having the acceptance defined by HPC clusters it is larger (about $0.04^{\circ}$ ). The latter sample has an additional contribution due to the poorer precision $\left( \pm 0.2^{\circ}\right)$ on the cluster polar angle determination. The total contribution to the acceptance definition depends on the fraction of events with HPC-based acceptance and it ranges from $\pm 0.24 \%$ to $\pm 0.30 \%$. Beam energy spread effects on the acceptance were investigated and they were found to be $\pm 0.28 \%$.

The corrections applied for the $\pm 2^{\circ}$ polar angle fiducial cut around $90^{\circ}$ in the analysis of the total and differential cross-sections were computed at the different energies by using the program TOPAZ0 and checked with ALIBABA. No significant differences were found between the two generators. An error of $\pm 0.25 \%$ was assigned to the total cross-section correlated between all energies. The rate of events with two same-charged particle tracks was compatible between data and simulation and consequently the charge misassignment was determined from simulated events. The charge misassignment error was determined by the statistics of the simulated events and it amounts to \pm 0.002 on the forward-backward asymmetry, correlated among all energies.

The TPC failure in the last part of the 2000 run had a clear influence on the Selection B efficiency: $63.0 \%$ instead of $75.5 \%$ obtained during the first part of the 2000 run. However the overall effect on the cross-sections and asymmetries was not very significant: the statistical error increase was of the order of a few percent and only an additional systematic error of $\pm 0.3 \%$ on the asymmetry was estimated because of the charge determination.

A breakdown of the systematic errors for data taken at $\sqrt{s} \sim 207 \mathrm{GeV}$ is given in Table 7.

\section{$5.2 \mu^{+} \mu^{-}$final states}

An analysis of $\mu^{+} \mu^{-}$final states at $\mathrm{e}^{+} \mathrm{e}^{-}$collision energies of $183 \mathrm{GeV}$ and above is presented. Data taken at energies of $183 \mathrm{GeV}$ and $189 \mathrm{GeV}$ [4] have been reanalysed to be consistent with data taken at higher energies. Compared to the previous analysis, the new analysis benefited from an increase in Monte Carlo statistics available in the whole LEP II data set. This made it possible to perform detailed comparisons of data and simulated events, which ultimately led to improvements in the estimation of the systematic errors on the measurements. For data taken at collision energies below $183 \mathrm{GeV}$ [3], the statistical errors are so large that improvements in the systematic errors would be negligible. These data have not been reanalysed.

\subsubsection{Analysis}

\section{Run selection}

Runs in which important components of the detector, such as the muon chambers, were not functioning satisfactorily were removed from the analysis.

\section{Event selection}

Events in the channel $\mathrm{e}^{+} \mathrm{e}^{-} \rightarrow \mu^{+} \mu^{-}$were selected by first imposing the following kinematic requirements:

- at least two reconstructed tracks from charged particles; 
- to allow for photon conversions or splitting of tracks, events with up to 7 reconstructed tracks were accepted;

- the measured momenta of the two highest-momentum reconstructed charged particles had to be greater than $15 \mathrm{GeV} / c$;

- the polar angles of the two highest-momentum reconstructed charged particles had to lie between $14^{\circ}$ and $166^{\circ}$;

- to suppress backgrounds from $\mathrm{e}^{+} \mathrm{e}^{-} \rightarrow \tau^{+} \tau^{-}$events, the momentum of the third highest-momentum charged particle had to be less than $5 \mathrm{GeV} / c$ in all cases where the energy of the leading charged particle was less than $90 \%$ of the beam energy.

Individual charged particles were identified as muons if:

- there was at least one hit in the muon chambers associated to the reconstructed track; or

- the associated energy deposits in the HCAL were consistent with a minimum ionising particle; or

- the associated energy in the electromagnetic calorimeters was less than $1.5 \mathrm{GeV}$.

The two highest-momentum charged particles had to be identified as muons. Events were rejected if:

- either of the two highest-momentum charged particles had no associated muon chambers hits and the energy per layer of the HCAL exceeded $5 \mathrm{GeV}$; or

- the electromagnetic energy associated to either of the two highest-momentum particles exceeded $10 \mathrm{GeV}$ and neither track had associated muon chamber hits - this removed $\mathrm{e}^{+} \mathrm{e}^{-} \rightarrow \mathrm{e}^{+} \mathrm{e}^{-}$events.

To reduce cosmic ray backgrounds, the reconstructed tracks of the two highest-momentum charged particles had to appear to come from the interaction region:

- for tracks which had associated VD hits, the modulus of the impact parameter relative to the beamspot in the plane transverse to the beam axis had to be less than $0.1 \mathrm{~cm}$, for at least one of the tracks;

- for tracks without hits in the VD, the modulus of the impact parameter had to be less than $1.0 \mathrm{~cm}$, for both tracks, reflecting the worse resolution for these tracks;

- in all cases, the impact parameter with respect to the beamspot in the direction parallel to the beam axis had to be less than $2.0 \mathrm{~cm}$, this cut being set by the length of the electron and positron bunches.

\section{$\sqrt{s^{\prime}}$ reconstruction}

Having selected suitable $\mathrm{e}^{+} \mathrm{e}^{-} \rightarrow \mu^{+} \mu^{-}$events, kinematic fits were used to determine the invariant mass of the $\mu^{+} \mu^{-}$pair, $\sqrt{s^{\prime}}$. The kinematic fits attempted to match the measured event to several possible event topologies, using energy and momentum constraints to improve the determination of the 3-momenta of the reconstructed muons. Use of the kinematic fits improved the resolution of the measured invariant masses of the $\mu^{+} \mu^{-}$pairs, compared to using the directly measured momenta of all muons, or using the measured angles of the muons, assuming a single photon emitted down the beampipe. As well as using the tracks selected as muons, up to one reconstructed photon per event was included in the fit. Electromagnetic energy clusters, not associated to any reconstructed track, were considered as photons if they had an energy exceeding $5 \mathrm{GeV}$.

The following kinematic fits were tried in the order given: 


\section{DELPHI}

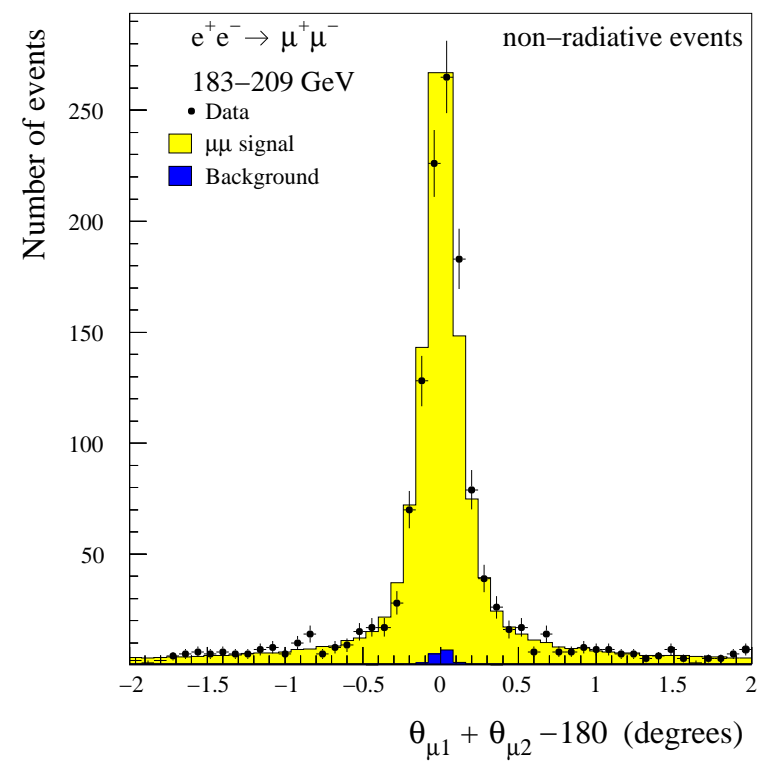

Figure 3: The distribution of $\theta_{\mu 1}+\theta_{\mu 2}-180^{\circ}$ for the two highest momentum muons in data and simulation. For back-to-back events the sum should be $0^{\circ}$. This is broadened by radiation, resolution and beam energy spread. Data from 199\%-2000 are shown.

- if a photon was found, the event was fitted assuming a seen photon and a possible additional unseen photon;

- a fit in which there was assumed to be no photons radiated in the event;

- a fit assuming a single photon emitted along the beampipe;

- a fit assuming a single photon emitted in any direction but unseen in the detector.

In each case, if the fit gave a satisfactory $\chi^{2}$ the invariant mass of the $\mu^{+} \mu^{-}$pair was calculated from the fitted momenta of the particles. Otherwise, the next fit was attempted. If none of the fits gave an acceptable $\chi^{2}$ the measured momenta of the muons were used to determine the invariant mass of the $\mu^{+} \mu^{-}$pair. Events were divided into inclusive and non-radiative classes according to the definitions given in the introduction to Section 5.

In the non-radiative class, migrations into and out of the sample were determined from the simulated events. The migrations amount to a correction of between $1.6 \%$ and $3.6 \%$ on the measured cross-sections.

\section{Estimation of the selection efficiency and background}

Selection efficiencies and backgrounds were determined from simulated events, but critical components of the analysis, such as the efficiency for reconstructing and identifying muons, were determined from the data. To obtain good agreement between the data and the predictions of simulations it was necessary to apply a number of corrections.

Using $\mathrm{e}^{+} \mathrm{e}^{-} \rightarrow \mu^{+} \mu^{-}$events, collected at $\sqrt{s} \sim \mathrm{M}_{\mathrm{Z}}$ at various times during 1997 through to 2000, smearings and shifts in the mean values for distributions of $1 / p$ were computed for different charges of particle, different ranges of $\cos \theta$, and different combinations of tracking detectors involved in the track reconstruction. These corrections were applied to data taken at LEP II energies, improving the agreement between data 
and simulations in the distribution of the momenta of particles at these higher centre-ofmass energies. Application of these corrections led to only small changes in the expected numbers of events selected in the inclusive and non-radiative classes. This reflects the fact that the relative weights given to measurements of angles in the kinematic fits were larger than the weights given to the less precisely measured momenta of particles.

In the simulations of $\mathrm{e}^{+} \mathrm{e}^{-} \rightarrow \mu^{+} \mu^{-}$events, the momenta of the incoming electrons and positrons were taken to be equal and opposite. However, in the data the particles in the incoming bunches of electrons and positrons had a momentum spread of $\sim 0.2$ $\mathrm{GeV} / c$, such that the energies of the electron and positron before collision were not necessarily equal. This led to an observable broadening of the acollinearity distribution at low acollinearities, in the data. By applying boosts to the simulated $\mu^{+} \mu^{-}$events, after the full detector simulation, reflecting the momentum spread of the real beams, this effect was taken into account. This led to improved agreement between data and simulation in distributions which were used to study the performance of the kinematic fits. The agreement between data and simulation after inclusion of the appropriate boosts is shown in Figure 3. Due to the relatively loose cuts in $\sqrt{s^{\prime}} / \sqrt{s}$, the application of the boosts in the simulation made a relatively small change to the expected number of events selected in the non-radiative sample.

Corrections were also applied to bring agreement between the data and simulation for the numbers of muon chamber hits associated to reconstructed tracks. These were determined from the high energy data themselves. Part of the corrections were to take into account unsimulated high voltage trips of the chambers which actually happened during the collection of the LEP II data set. Because the muon identification criteria select events from a union of 3 highly overlapping samples, application of the correction had only a limited impact on the expected numbers of particles identified as muons.

After applying the corrections above, differences between data and simulation were still found in the efficiency to reconstruct and identify single muons. High purity samples of single muons with high momentum were selected, and the efficiency to reconstruct and identify a single muon was determined from the number of times a second muon was found accompanying the first muon. The backgrounds from events other than $\mathrm{e}^{+} \mathrm{e}^{-} \rightarrow \mu^{+} \mu^{-}$ events, such as $\mathrm{e}^{+} \mathrm{e}^{-} \rightarrow \tau^{+} \tau^{-}$and $\mathrm{e}^{+} \mathrm{e}^{-} \rightarrow \mathrm{W}^{+} \mathrm{W}^{-}$which could lead to only one muon and one other track in the detector, were negligible. Averaging over all data from $\sqrt{s} \sim$ $183 \mathrm{GeV}$ and above, it was found that the efficiency for selecting $\mathrm{e}^{+} \mathrm{e}^{-} \rightarrow \mu^{+} \mu^{-}$events determined from the simulation had to be reduced by $(1.08 \pm 0.49) \%$. The error on this value, which is dominated by statistics of events selected in data, is the most significant systematic error for the measurement of the non-radiative cross-sections, and is correlated between measurements at all energies.

The efficiency for selecting $\mathrm{e}^{+} \mathrm{e}^{-} \rightarrow \mu^{+} \mu^{-}$events determined from the simulation was also corrected for the efficiency for triggering DELPHI on these events. This was estimated using the redundancy of the DELPHI trigger system in selected $\mathrm{e}^{+} \mathrm{e}^{-} \rightarrow \mu^{+} \mu^{-}$ events. It was found to be stable over all years of data taking. Averaging over all years the efficiency was determined to be $(99.82 \pm 0.07) \%$. No significant variation in efficiency was found over $\cos \theta$ within the acceptance of the analysis.

Backgrounds from $\mathrm{e}^{+} \mathrm{e}^{-} \rightarrow \tau^{+} \tau^{-}, \mathrm{e}^{+} \mathrm{e}^{-} \rightarrow \mathrm{W}^{+} \mathrm{W}^{-}, \mathrm{e}^{+} \mathrm{e}^{-} \rightarrow \mathrm{ZZ}$ and $\mathrm{e}^{+} \mathrm{e}^{-} \rightarrow \mathrm{e}^{+} \mathrm{e}^{-} \mathrm{f} \overline{\mathrm{f}}$ processes were estimated from samples of simulated events. Backgrounds from $\mathrm{e}^{+} \mathrm{e}^{-} \rightarrow \mathrm{e}^{+} \mathrm{e}^{-}, \mathrm{e}^{+} \mathrm{e}^{-} \rightarrow \mathrm{q} \overline{\mathrm{q}}$ and two-photon collisions were estimated to be negligible. The background from cosmic rays inside the selected sample was estimated by extrapolating the numbers of cosmic rays failing the cosmic ray rejection cuts, from within a looser selec- 


\begin{tabular}{|l|c|c|}
\hline \multicolumn{3}{|c|}{$\mathrm{e}^{+} \mathrm{e}^{-} \rightarrow \mu^{+} \mu^{-}$} \\
\hline \hline $\begin{array}{l}\text { Background } \\
\text { source }\end{array}$ & $\begin{array}{c}\text { Non-radiative } \\
\text { background }\end{array}$ & $\begin{array}{c}\text { Inclusive } \\
\text { background }\end{array}$ \\
\hline \hline $\mathrm{e}^{+} \mathrm{e}^{-} \rightarrow \tau^{+} \tau^{-}$ & $0.08 \pm 0.02$ & $0.94 \pm 0.03$ \\
$\mathrm{e}^{+} \mathrm{e}^{-} \rightarrow \mathrm{W}^{+} \mathrm{W}^{-}$ & $0.16 \pm 0.07$ & $3.56 \pm 0.21$ \\
$\mathrm{e}^{+} \mathrm{e}^{-} \rightarrow \mathrm{ZZ}$ & $0.26 \pm 0.06$ & $1.01 \pm 0.06$ \\
$\mathrm{e}^{+} \mathrm{e}^{-} \rightarrow \mathrm{e}^{+} \mathrm{e}^{-} \mathrm{ff}$ & $0.10 \pm 0.06$ & $3.75 \pm 0.59$ \\
Cosmic rays & $0.44 \pm 0.04$ & $0.45 \pm 0.04$ \\
\hline \hline Total & $1.04 \pm 0.12$ & $9.72 \pm 0.63$ \\
\hline
\end{tabular}

Table 8: Residual background levels for data taken at $\sqrt{s} \sim 207$ GeV (in \% relative to the number of selected $\mathrm{e}^{+} \mathrm{e}^{-} \rightarrow \mu^{+} \mu^{-}$candidates).

\section{DELPHI}

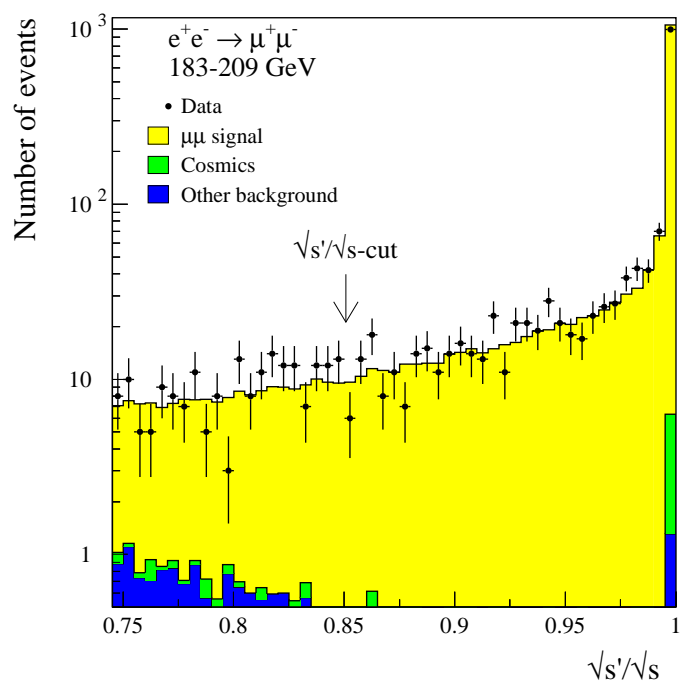

DELPHI

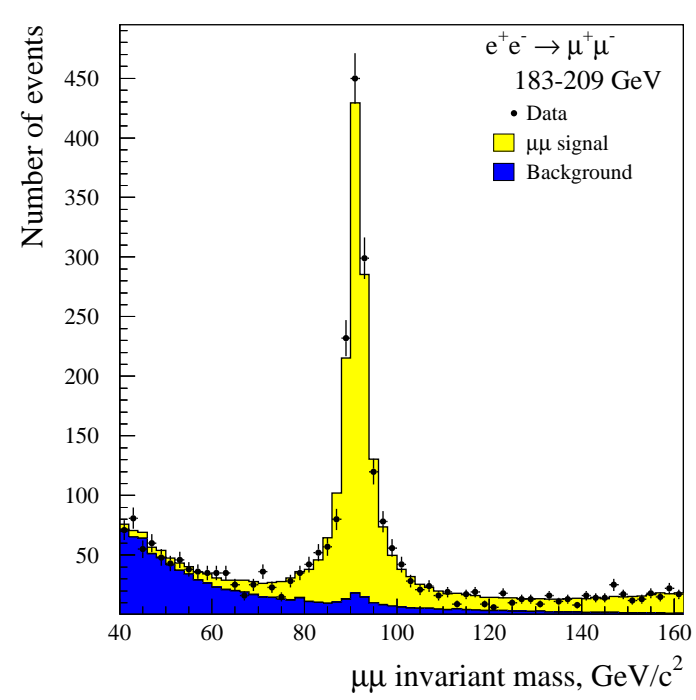

Figure 4: The reconstructed $\sqrt{s^{\prime}} / \sqrt{s}$ distribution (left) and the reconstructed $\sqrt{s^{\prime}}$ distribution (right) for $\mathrm{e}^{+} \mathrm{e}^{-} \rightarrow \mu^{+} \mu^{-}$events. Both plots show data from 1997-2000. In the right hand plot all selection criteria except for the cut on the $\mu^{+} \mu^{-}$invariant mass were applied.

tion on the impact parameters of the reconstructed tracks into the selected $\mathrm{e}^{+} \mathrm{e}^{-} \rightarrow \mu^{+} \mu^{-}$ region. The estimated backgrounds are given in Table 8 .

The distributions of $\sqrt{s^{\prime}} / \sqrt{s}$ for events in the non-radiative region and $\sqrt{s^{\prime}}$ in the radiative return region are shown in Figure 4 . There is good agreement between data and simulation, including in the low mass region dominated by backgrounds.

\subsubsection{Results}

In total 3684 events were selected in the inclusive sample and 1595 were selected in the non-radiative class from data at the $183-207 \mathrm{GeV}$ energy points. For the non- 
radiative sample the average efficiency in the fiducial volume, taking into account all corrections, was $93.25 \pm 0.48 \%$, and the background from non $\mathrm{e}^{+} \mathrm{e}^{-} \rightarrow \mu^{+} \mu^{-}$collisions was estimated to increase from $(10 \pm 2) \mathrm{fb}$ at $\sqrt{s} \sim 183 \mathrm{GeV}$ to $(13 \pm 3) \mathrm{fb}$ at $\sqrt{s} \sim$ $207 \mathrm{GeV}$, which is at most approximately $0.6 \%$ of the signal cross-section. In the inclusive sample the total background amounted to, at most, $9 \%$ of the signal cross-section. The dominant backgrounds came from four-fermion events which were misidentified as twofermion events. Cosmic ray events were estimated to account for between $0.31 \%$ and $0.45 \%$ of the selected events. The efficiency, background etc. at each energy is given in Table 9. The selected events were used to measure the total cross-sections, forwardbackward asymmetries and the differential cross-sections for non-radiative events.

\section{Cross-sections}

The total cross-section for the non-radiative and inclusive samples of $\mathrm{e}^{+} \mathrm{e}^{-} \rightarrow \mu^{+} \mu^{-}$ events was computed from

$$
\sigma_{\mu \mu}=\eta_{4 \pi} \frac{\left(N_{s e l}-N_{b g}\right)(1-f)}{\epsilon \mathcal{L}}
$$

where $N_{\text {sel }}$ is the numbers of events selected in each sample, $N_{b g}$ is the predicted number of background events selected in the sample for the luminosity $\mathcal{L}$. The efficiency for selecting $\mathrm{e}^{+} \mathrm{e}^{-} \rightarrow \mu^{+} \mu^{-}$events in the sample is $\epsilon$, which includes corrections for the efficiency to reconstruct and identify muons and the trigger efficiency, obtained from the data. $f$ (applicable to the non-radiative cross-section only) is a factor for the migration of $\mathrm{e}^{+} \mathrm{e}^{-} \rightarrow \mu^{+} \mu^{-}$events into the sample from lower centre-of-mass energy. $\eta_{4 \pi}$ is a correction factor required to extrapolate the measurements from the polar angular acceptance to the full $4 \pi$ acceptance, determined from simulated events. The measured cross-sections are given in Table 10 and shown in Figure 10.

For the $207 \mathrm{GeV}$ energy point separate measurements were made for the periods before and after the failure of the sector of the TPC. These measured cross-sections were combined using the BLUE technique [29] which performs a weighted average of the measurements taking into account correlated errors.

\section{Forward-backward asymmetries}

The forward-backward asymmetry for each sample was computed from

$$
\mathrm{A}_{\mathrm{FB}}=\frac{N_{f}-c_{b} N_{b}}{N_{f}+c_{b} N_{b}}
$$

where $N_{f}$ and $N_{b}$ are the numbers of $\mathrm{e}^{+} \mathrm{e}^{-} \rightarrow \mu^{+} \mu^{-}$events in which the $\mu^{-}$is in the forward and backward hemispheres defined as $\cos \theta>0$ and $\cos \theta<0$ respectively. Differences in the efficiencies for selecting events and the migration of events into the non-radiative samples in the forward and backward hemispheres are accounted for by the factor $c_{b}$, which was determined for each sample from simulated events. These differences arise from the different fractions of non-radiative and radiative return events in the forward and backward hemispheres reflecting the very different forward-backward asymmetries of these subsamples. $N_{f}$ and $N_{b}$ are computed from

$$
N_{f / b}=\eta_{2 \pi}\left(N_{\mu^{+} \mu^{-}}^{f / b}-N_{b g}^{f / b}+N_{ \pm}^{f / b}\right),
$$

where $N_{\mu^{+} \mu^{-}}^{f / b}$ and $N_{b g}^{f / b}$ are the numbers of events selected and the predicted backgrounds in each hemisphere. $\eta_{2 \pi}$ is a factor to extrapolate the observed number of events in 


\begin{tabular}{|l|c|c|c|c|c|c|c|c|}
\hline \multicolumn{10}{|c|}{$\mathrm{e}^{+} \mathrm{e}^{-} \rightarrow \mu^{+} \mu^{-}$} \\
\hline \hline & 183 & 189 & 192 & 196 & 200 & 202 & 205 & 207 \\
\cline { 2 - 9 } & 182.65 & 188.56 & 191.60 & 195.53 & 199.53 & 201.65 & 204.85 & 206.55 \\
\hline \hline Energy (GeV) & 52.54 & 156.38 & 25.79 & 73.98 & 83.14 & 40.51 & 75.55 & 137.07 \\
Lumi $\left(\mathrm{pb}^{-1}\right)$ & 379 & 991 & 167 & 389 & 506 & 205 & 373 & 674 \\
No. Events & 92.7 & 93.7 & 93.1 & 93.5 & 93.2 & 93.2 & 93.6 & 92.7 \\
Efficiency (\%) & 0.7 & 0.9 & 0.8 & 0.8 & 0.8 & 0.9 & 1.1 & 1.0 \\
Background (\%) & 0.9 & \multicolumn{10}{|c|}{ Energy point $(\mathrm{GeV})$} \\
\hline
\end{tabular}

Table 9: Details of LEP II analysis for the $\mathrm{e}^{+} \mathrm{e}^{-} \rightarrow \mu^{+} \mu^{-}$channel. The table shows the actual centre-of-mass energy and luminosity analysed at each energy point, the number of events selected in the inclusive analysis and the efficiencies for selecting events in the non-radiative samples and the backgrounds selected in the non-radiative samples.

\begin{tabular}{|c|c|c|c|c|}
\hline \multicolumn{5}{|c|}{$\mathrm{e}^{+} \mathrm{e}^{-} \rightarrow \mu^{+} \mu^{-}$} \\
\hline \multirow{2}{*}{$\begin{array}{c}\sqrt{s} \\
(\mathrm{GeV})\end{array}$} & \multicolumn{2}{|c|}{$\sqrt{s^{\prime}}>75(\mathrm{GeV})$} & \multicolumn{2}{|c|}{$\sqrt{s^{\prime}} / \sqrt{s}>0.85$} \\
\hline & $\sigma_{\mu \mu}(\mathrm{pb})$ & $\mathrm{A}_{\mathrm{FB}}^{\mu}$ & $\sigma_{\mu \mu}(\mathrm{pb})$ & $\mathrm{A}_{\mathrm{FB}}^{\mu}$ \\
\hline 130 & $\begin{array}{c}24.30 \pm 3.20 \pm 0.83 \\
(20.04)\end{array}$ & $\begin{array}{c}0.450 \pm 0.120 \pm 0.002 \\
(0.339)\end{array}$ & $\begin{array}{c}9.70 \pm 1.90 \pm 0.36 \\
(8.11)\end{array}$ & $\begin{array}{c}0.670 \pm 0.150 \pm 0.003 \\
(0.719)\end{array}$ \\
\hline 136 & $\begin{array}{c}17.00 \pm 2.60 \pm 0.58 \\
(17.07)\end{array}$ & $\begin{array}{c}0.560 \pm 0.130 \pm 0.002 \\
(0.340)\end{array}$ & $\begin{array}{c}6.60 \pm 1.60 \pm 0.25 \\
(7.00)\end{array}$ & $\begin{array}{c}0.740 \pm 0.160 \pm 0.003 \\
(0.699)\end{array}$ \\
\hline 161 & $\begin{array}{c}9.30 \pm 1.10 \pm 0.32 \\
(10.32)\end{array}$ & $\begin{array}{c}0.390 \pm 0.110 \pm 0.002 \\
(0.335)\end{array}$ & $\begin{array}{c}3.60 \pm 0.70 \pm 0.13 \\
(4.43)\end{array}$ & $\begin{array}{c}0.430 \pm 0.160 \pm 0.003 \\
(0.629)\end{array}$ \\
\hline 172 & $\begin{array}{c}8.90 \pm 1.10 \pm 0.31 \\
(8.74)\end{array}$ & $\begin{array}{c}0.550 \pm 0.100 \pm 0.002 \\
(0.332)\end{array}$ & $\begin{array}{c}3.60 \pm 0.70 \pm 0.12 \\
(3.79)\end{array}$ & $\begin{array}{c}0.940 \pm 0.140 \pm 0.003 \\
(0.610)\end{array}$ \\
\hline 183 & $\begin{array}{c}8.28 \pm 0.46 \pm 0.07 \\
(7.56)\end{array}$ & $\begin{array}{c}0.299 \pm 0.051 \pm 0.003 \\
(0.329)\end{array}$ & $\begin{array}{c}3.61 \pm 0.28 \pm 0.03 \\
(3.30)\end{array}$ & $\begin{array}{c}0.588 \pm 0.064 \pm 0.001 \\
(0.596)\end{array}$ \\
\hline 189 & $\begin{array}{c}7.17 \pm 0.24 \pm 0.06 \\
(7.01)\end{array}$ & $\begin{array}{c}0.336 \pm 0.032 \pm 0.003 \\
(0.327)\end{array}$ & $\begin{array}{c}3.07 \pm 0.15 \pm 0.02 \\
(3.07)\end{array}$ & $\begin{array}{c}0.600 \pm 0.039 \pm 0.001 \\
(0.589)\end{array}$ \\
\hline 192 & $\begin{array}{c}7.37 \pm 0.61 \pm 0.07 \\
(6.76)\end{array}$ & $\begin{array}{c}0.275 \pm 0.078 \pm 0.003 \\
(0.327)\end{array}$ & $\begin{array}{c}2.82 \pm 0.36 \pm 0.02 \\
(2.97)\end{array}$ & $\begin{array}{c}0.636 \pm 0.098 \pm 0.001 \\
(0.586)\end{array}$ \\
\hline 196 & $\begin{array}{c}5.89 \pm 0.32 \pm 0.06 \\
(6.45)\end{array}$ & $\begin{array}{c}0.297 \pm 0.052 \pm 0.004 \\
(0.326)\end{array}$ & $\begin{array}{c}2.76 \pm 0.21 \pm 0.02 \\
(2.84)\end{array}$ & $\begin{array}{c}0.586 \pm 0.061 \pm 0.001 \\
(0.582)\end{array}$ \\
\hline 200 & $\begin{array}{c}6.95 \pm 0.33 \pm 0.07 \\
(6.16)\end{array}$ & $\begin{array}{c}0.334 \pm 0.044 \pm 0.003 \\
(0.324)\end{array}$ & $\begin{array}{c}3.08 \pm 0.21 \pm 0.02 \\
(2.71)\end{array}$ & $\begin{array}{c}0.548 \pm 0.056 \pm 0.001 \\
(0.578)\end{array}$ \\
\hline 202 & $\begin{array}{c}5.70 \pm 0.44 \pm 0.06 \\
(6.02)\end{array}$ & $\begin{array}{c}0.365 \pm 0.070 \pm 0.004 \\
(0.324)\end{array}$ & $\begin{array}{c}2.46 \pm 0.27 \pm 0.02 \\
(2.65)\end{array}$ & $\begin{array}{c}0.544 \pm 0.090 \pm 0.001 \\
(0.577)\end{array}$ \\
\hline 205 & $\begin{array}{c}5.46 \pm 0.31 \pm 0.06 \\
(5.81)\end{array}$ & $\begin{array}{c}0.304 \pm 0.053 \pm 0.004 \\
(0.323)\end{array}$ & $\begin{array}{c}2.35 \pm 0.19 \pm 0.02 \\
(2.56)\end{array}$ & $\begin{array}{c}0.642 \pm 0.061 \pm 0.001 \\
(0.574)\end{array}$ \\
\hline 207 & $\begin{array}{c}5.49 \pm 0.23 \pm 0.06 \\
(5.70)\end{array}$ & $\begin{array}{c}0.373 \pm 0.039 \pm 0.006 \\
(0.323)\end{array}$ & $\begin{array}{c}2.47 \pm 0.15 \pm 0.02 \\
(2.52)\end{array}$ & $\begin{array}{c}0.558 \pm 0.048 \pm 0.001 \\
(0.573)\end{array}$ \\
\hline
\end{tabular}

Table 10: Measured cross-sections and forward-backward asymmetries for inclusive and non-radiative $\mathrm{e}^{+} \mathrm{e}^{-} \rightarrow \mu^{+} \mu^{-}$events. The first uncertainty is statistical, the second systematic. Numbers in brackets are the theoretical predictions of ZFITTER, which are estimated to have a precision of $\pm 0.4 \%$ on $\sigma_{\mu \mu}$ and \pm 0.004 on $\mathrm{A}_{\mathrm{FB}}^{\mu}$. 
the angular acceptance to $0 \leq|\cos \theta| \leq 1 . N_{ \pm}^{f / b}$ account for the expected numbers of events in each hemisphere with misidentified charges, such that an event in which the $\mu^{-}$was produced in the forward hemisphere is reconstructed as an event with the $\mu^{-}$in the backward hemisphere and vice versa. There were approximately $0.5 \%$ of events in which the charge of one of the particles was misidentified, so that both particles were reconstructed with the same charge. In these cases, the particle with the larger relative uncertainty on its momentum was considered to have its charge mismeasured and its charge was inverted before the event was assigned to either the forward or backward hemisphere. From simulation this was found to identify the wrongly measured charge in $93 \pm 5 \%$ of cases. The rate of events in which both particles had their charges mismeasured was found to be negligible. The measured forward-backward asymmetries are given in Table 10 and shown in Figure 11.

For the $207 \mathrm{GeV}$ energy point, separate measurements of the numbers of events in the forward and backward hemispheres were made for the periods before and after the failure of the sector of the TPC. These measurements were added together and an average forward-backward asymmetry was computed.

\section{Differential cross-sections}

The differential cross-sections, $\mathrm{d} \sigma / \mathrm{d} \cos \theta$ for the non-radiative samples of $\mathrm{e}^{+} \mathrm{e}^{-} \rightarrow \mu^{+} \mu^{-}$events were computed in 10 bins, $i$, of $\cos \theta$ in the range $-0.970<\cos \theta<$ 0.970 using

$$
\mathrm{d} \sigma / \mathrm{d} \cos \theta_{i}=\frac{\eta_{i} f^{i}\left(N_{\mu^{+} \mu^{-}}^{i}-N_{b g}^{i}+N_{ \pm}^{i}\right)}{\epsilon_{i} \mathcal{L} \Delta_{i}},
$$

where $N_{\mu^{+} \mu^{-}}^{i}$ and $N_{b g}^{i}$ are the number of observed events and the expected number of background events in each bin of $\cos \theta$, where $\theta$ is the polar angle of the negatively charged muon with respect to the beam axis. $\epsilon_{i}$ is the efficiency in each bin of $\cos \theta$, which was corrected, in all bins, for the global track reconstruction and muon identification correction factor determined for the total cross-sections and the trigger efficiency. The bin width in $\cos \theta$ is $\Delta_{i}$. The migration factor between non-radiative and radiative return events, $f$, was computed individually for each bin from simulated events

Migrations between neighbouring bins of $\cos \theta$ were found to be negligible. The expected number of events with mismeasured charges in each bin was accounted for by $N_{ \pm}^{i}$.

Measurements in the outer bins of $\cos \theta$ were not corrected to the full angular acceptance. Corrections were made for the experimental cuts on the polar angle of both muons by the factor $\eta$ which was computed from simulated events and found to be significant only in the bins with largest $|\cos \theta|$. The measured differential cross-sections are given in Table 11.

For the $207 \mathrm{GeV}$ energy point separate measurements of the differential cross-sections in each bin were made for the periods before and after the failure of the sector of the TPC. The measurements were combined using the BLUE technique, taking into account correlated systematic errors.

\section{Systematic errors}

For measurements of the non-radiative cross-sections the largest single systematic error comes from the determination of the selection efficiency which is dominated by the correction factor for muon identification and track reconstruction efficiencies. The 


\begin{tabular}{|c|c|c|c|c|c|c|c|c|}
\hline \multicolumn{9}{|c|}{$\mathrm{e}^{+} \mathrm{e}^{-} \rightarrow \mu^{+} \mu^{-}$} \\
\hline \multicolumn{3}{|c|}{$\sqrt{s} \sim 183$} & \multicolumn{3}{|c|}{$\sqrt{s} \sim 189$} & \multicolumn{3}{|c|}{$\sqrt{s} \sim 192$} \\
\hline \multirow[b]{2}{*}{$\cos \theta$} & \multicolumn{2}{|r|}{$\mathrm{d} \sigma / \mathrm{d} \cos \theta(\mathrm{pb})$} & \multirow[b]{2}{*}{$\cos \theta$} & \multicolumn{2}{|r|}{$\mathrm{d} \sigma / \mathrm{d} \cos \theta(\mathrm{pb})$} & \multirow[b]{2}{*}{$\cos \theta$} & \multicolumn{2}{|r|}{$\mathrm{d} \sigma / \mathrm{d} \cos \theta(\mathrm{pb})$} \\
\hline & $\mathrm{SM}$ & Measurement & & $\mathrm{SM}$ & Measurement & & SM & Measurement \\
\hline$[-0.97,-0.80]$ & 0.47 & $-0.01 \pm 0.00(0.24) \pm 0.00$ & {$[-0.97,-0.80]$} & 0.46 & $0.41 \pm 0.13(0.14) \pm 0.01$ & {$[-0.97,-0.80]$} & 0.45 & $-0.01 \pm 0.00(0.33) \pm 0.00$ \\
\hline$[-0.80,-0.60]]$ & 0.48 & $0.41 \pm 0.21(0.22) \pm 0.01$ & {$[-0.80,-0.60]$} & 0.46 & $0.47 \pm 0.13(0.12) \pm 0.01$ & {$[-0.80,-0.60]$} & 0.45 & $0.21 \pm 0.21(0.31) \pm 0.00$ \\
\hline$[-0.60,-0.40]$ & 0.57 & $1.00 \pm 0.32(0.24) \pm 0.01$ & {$[-0.60,-0.40]$} & 0.54 & $0.54 \pm 0.14(0.14) \pm 0.01$ & {$[-0.60,-0.40]$} & 0.53 & $0.83 \pm 0.42(0.33) \pm 0.01$ \\
\hline$[-0.40,-0.20]$ & 0.76 & $1.06 \pm 0.33(0.27) \pm 0.01$ & {$[-0.40,-0.20]$} & 0.71 & $0.43 \pm 0.12(0.16) \pm 0.01$ & {$[-0.40,-0.20]$} & 0.69 & $0.42 \pm 0.30(0.38) \pm 0.01$ \\
\hline$[-0.20,0.00]$ & 1.05 & $1.14 \pm 0.35(0.33) \pm 0.01$ & {$[-0.20,0.00]$} & 0.98 & $1.21 \pm 0.21(0.19) \pm 0.02$ & {$[-0.20,0.00]$} & 0.94 & $1.09 \pm 0.49(0.45) \pm 0.01$ \\
\hline $0.00,0.20]$ & 1.44 & $1.69 \pm 0.43(0.39) \pm 0.02$ & {$[0.00,0.20]$} & 1.33 & $1.25 \pm 0.21(0.22) \pm 0.02$ & {$[0.00,0.20]$} & 1.28 & $0.67 \pm 0.40(0.54) \pm 0.01$ \\
\hline $0.20,0.40]$ & 1.92 & $1.79 \pm 0.43(0.44) \pm 0.02$ & $0.20,0.40]$ & 1.78 & $2.03 \pm 0.26(0.24) \pm 0.02$ & $0.20,0.40]$ & 1.71 & $2.98 \pm 0.80(0.60) \pm 0.02$ \\
\hline $0.40,0.60]$ & 2.52 & $3.34 \pm 0.59(0.51) \pm 0.03$ & $0.40,0.60]$ & 2.33 & $2.19 \pm 0.28(0.28) \pm 0.02$ & $0.40,0.60]$ & 2.24 & $2.27 \pm 0.69(0.68) \pm 0.02$ \\
\hline $0.60,0.80]$ & 3.22 & $3.30 \pm 0.60(0.58) \pm 0.03$ & $0.60,0.80]$ & 2.98 & $2.86 \pm 0.32(0.32) \pm 0.03$ & $0.60,0.80]$ & 2.87 & $2.30 \pm 0.70(0.77) \pm 0.02$ \\
\hline $0.80,0.97]$ & 4.00 & $4.07 \pm 0.72(0.71) \pm 0.04$ & $0.80,0.97]$ & 3.70 & $3.90 \pm 0.40(0.39) \pm 0.04$ & $0.80,0.97]$ & 3.57 & $3.22 \pm 0.90(0.94) \pm 0.03$ \\
\hline \multicolumn{3}{|c|}{$\sqrt{s} \sim 196$} & \multicolumn{3}{|c|}{$\sqrt{s} \sim 200$} & \multicolumn{3}{|c|}{$\sqrt{s} \sim 202$} \\
\hline \multirow[b]{2}{*}{$\cos \theta$} & \multicolumn{2}{|r|}{ 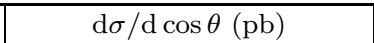 } & \multirow[b]{2}{*}{$\cos \theta$} & \multicolumn{2}{|r|}{ 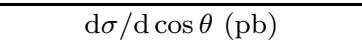 } & \multicolumn{3}{|r|}{$\overline{\mathrm{d} \sigma} \sigma / \mathrm{d} \cos \theta(\mathrm{pb})$} \\
\hline & SM & Measurement & & $\mathrm{SM}$ & Measurement & $\cos \theta$ & $\mathrm{SM}$ & Measurement \\
\hline$[-0.97,-0.80]$ & 0.44 & $0.68 \pm 0.24(0.19) \pm 0.01$ & 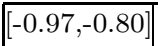 & 0.43 & $0.53 \pm 0.20(0.18) \pm 0.01$ & {$[-0.97,-0.80]$} & 0.43 & $1.43 \pm 0.48(0.26) \pm 0.02$ \\
\hline$[-0.80,-0.60]$ & 0.44 & $0.51 \pm 0.19(0.18) \pm 0.01$ & {$[-0.80,-0.60]$} & 0.43 & $0.26 \pm 0.13(0.17) \pm 0.00$ & {$[-0.80,-0.60]$} & 0.42 & $0.54 \pm 0.27(0.24) \pm 0.01$ \\
\hline$[-0.60,-0.40]$ & 0.51 & $0.65 \pm 0.22(0.19) \pm 0.01$ & {$[-0.60,-0.40]$} & 0.49 & $0.83 \pm 0.23(0.18) \pm 0.01$ & {$[-0.60,-0.40]$} & 0.49 & $0.25 \pm 0.18(0.25) \pm 0.00$ \\
\hline$[-0.40,-0.20]$ & 0.66 & $0.22 \pm 0.13(0.22) \pm 0.01$ & {$[-0.40,-0.20]$} & 0.64 & $0.71 \pm 0.22(0.20) \pm 0.01$ & {$[[-0.40,-0.20]$} & 0.62 & $0.26 \pm 0.19(0.29) \pm 0.01$ \\
\hline$[-0.20,0.00]$ & 0.90 & $0.84 \pm 0.26(0.26) \pm 0.01$ & {$[-0.20,0.00]$} & 0.86 & $1.10 \pm 0.28(0.24) \pm 0.01$ & {$[-0.20,0.00]$} & 0.84 & $0.56 \pm 0.28(0.34) \pm 0.01$ \\
\hline$[0.00,0.20]$ & 1.22 & $1.71 \pm 0.37(0.31) \pm 0.02$ & {$[0.00,0.20]$} & 1.17 & $1.44 \pm 0.32(0.28) \pm 0.01$ & {$[0.00,0.20]$} & 1.14 & $0.71 \pm 0.32(0.40) \pm 0.01$ \\
\hline$[0.20,0.40]$ & 1.63 & $1.33 \pm 0.32(0.35) \pm 0.01$ & $0.20,0.40]$ & 1.56 & $1.66 \pm 0.33(0.32) \pm 0.02$ & $0.20,0.40]$ & 1.52 & $2.45 \pm 0.58(0.45) \pm 0.02$ \\
\hline$[0.40,0.60]$ & 2.14 & $2.08 \pm 0.39(0.39) \pm 0.02$ & $0.40,0.60]$ & 2.04 & $2.72 \pm 0.42(0.36) \pm 0.02$ & $0.40,0.60]$ & 1.99 & $1.45 \pm 0.44(0.51) \pm 0.02$ \\
\hline$[0.60,0.80]$ & 2.74 & $2.92 \pm 0.46(0.45) \pm 0.02$ & $0.60,0.80]$ & 2.61 & $2.80 \pm 0.43(0.41) \pm 0.02$ & $0.60,0.80]$ & 2.55 & $1.87 \pm 0.50(0.58) \pm 0.02$ \\
\hline $0.80,0.97]$ & 3.41 & $2.75 \pm 0.49(0.54) \pm 0.03$ & {$[0.80,0.97]$} & 3.25 & $3.18 \pm 0.50(0.50) \pm 0.03$ & {$[0.80,0.97]$} & 3.18 & $3.14 \pm 0.71(0.71) \pm 0.03$ \\
\hline \multicolumn{3}{|c|}{$\sqrt{s} \sim 205$} & & & & \multicolumn{3}{|c|}{$\sqrt{s} \sim 207$} \\
\hline \multirow[b]{2}{*}{$\cos \theta$} & \multicolumn{2}{|r|}{$\mathrm{d} \sigma / \mathrm{d} \cos \theta(\mathrm{pb})$} & & & & \multirow[b]{2}{*}{$\cos \theta$} & \multicolumn{2}{|r|}{$\mathrm{d} \sigma / \mathrm{d} \cos \theta(\mathrm{pb})$} \\
\hline & SM & Measurement & & & & & $\mathrm{SM}$ & Measurement \\
\hline$[-0.97,-0.80]$ & 0.42 & $0.67 \pm 0.24(0.19) \pm 0.01$ & & & & {$[-0.97,-0.80]$} & 0.42 & $0.47 \pm 0.15(0.14) \pm 0.01$ \\
\hline$[-0.80,-0.60]$ & 0.42 & $0.35 \pm 0.16(0.17) \pm 0.00$ & & & & {$[-0.80,-0.60]$} & 0.41 & $0.47 \pm 0.13(0.13) \pm 0.00$ \\
\hline$[-0.60,-0.40]$ & 0.47 & $0.20 \pm 0.12(0.18) \pm 0.00$ & & & & {$[-0.60,-0.40]$} & 0.47 & $0.42 \pm 0.13(0.13) \pm 0.00$ \\
\hline$[-0.40,-0.20]$ & 0.60 & $0.34 \pm 0.16(0.20) \pm 0.01$ & & & & {$[-0.40,-0.20]$} & 0.59 & $0.54 \pm 0.14(0.15) \pm 0.01$ \\
\hline$[-0.20,0.00]$ & 0.81 & $0.57 \pm 0.20(0.24) \pm 0.01$ & & & & {$[-0.20,0.00]$} & 0.80 & $0.71 \pm 0.16(0.18) \pm 0.01$ \\
\hline$[0.00,0.20]$ & 1.10 & $0.95 \pm 0.27(0.28) \pm 0.01$ & & & & {$[0.00,0.20]$} & 1.08 & $1.00 \pm 0.20(0.21) \pm 0.01$ \\
\hline$[0.20,0.40]$ & 1.47 & $1.33 \pm 0.31(0.32) \pm 0.01$ & & & & {$[0.20,0.40]$} & 1.44 & $1.39 \pm 0.23(0.24) \pm 0.01$ \\
\hline$[0.40,0.60]$ & 1.92 & $1.40 \pm 0.32(0.37) \pm 0.02$ & & & & $0.40,0.60]$ & 1.88 & $2.61 \pm 0.32(0.27) \pm 0.02$ \\
\hline$[0.60,0.80]$ & 2.46 & $2.16 \pm 0.40(0.42) \pm 0.02$ & & & & $0.60,0.80]$ & 2.41 & $1.93 \pm 0.28(0.31) \pm 0.02$ \\
\hline$[0.80,0.97]$ & 3.06 & $4.23 \pm 0.61(0.51) \pm 0.03$ & & & & {$[0.80,0.97]$} & 3.01 & $2.65 \pm 0.36(0.38) \pm 0.02$ \\
\hline
\end{tabular}

Table 11: Differential cross-sections for non-radiative $\mathrm{e}^{+} \mathrm{e}^{-} \rightarrow \mu^{+} \mu^{-}$events at centre-of-mass energies from 183 to $207 \mathrm{GeV}$. The tables show the bins, the predictions of the Standard Model and the measurements. The errors quoted are the statistical and experimental systematic errors. The statistical errors are shown as the measured errors and, in brackets, the expected errors, computed from the square root of the observed and expected numbers of events respectively. 


\begin{tabular}{|l|c|c||c|c|}
\hline \multicolumn{5}{|c|}{$\mathrm{e}^{+} \mathrm{e}^{-} \rightarrow \mu^{+} \mu^{-}$} \\
\hline \hline Source & $\begin{array}{c}\Delta \sigma / \sigma \\
\text { (non-rad.) }\end{array}$ & $\begin{array}{c}\Delta \sigma / \sigma \\
\text { (inclus.) }\end{array}$ & $\begin{array}{c}\Delta A_{F B} \\
\text { (non-rad.) }\end{array}$ & $\begin{array}{c}\Delta A_{F B} \\
\text { (inclus.) }\end{array}$ \\
\hline \hline Efficiency & 51 & 51 & 9 & 20 \\
$\sqrt{s^{\prime}}$ reconstruction & 14 & 20 & 2 & 0 \\
Charge misidentification & - & - & 6 & 4 \\
QED & 2 & 9 & 1 & 0 \\
Backgrounds & 12 & 70 & 6 & 51 \\
Luminosity & 55 & 55 & - & - \\
\hline \hline Total uncertainty & 77 & 105 & 12 & 55 \\
\hline Correlated & 75 & 103 & 11 & 53 \\
Uncorrelated & 16 & 17 & 5 & 17 \\
\hline
\end{tabular}

Table 12: Systematic uncertainties on the measured cross-sections and forward-backward asymmetries for data taken at $\sqrt{s} \sim 207 \mathrm{GeV}$. All numbers in units of $10^{-4}$. Separate measurements were made before and after the TPC sector failure, and then combined. There is thus no explicit systematic uncertainty related to the TPC sector instability. The correlated error component includes errors correlated between energies and channels and those correlated with other LEP experiments. 
relative error on the correction factor is $0.49 \%$ and is correlated between all energies and between all bins in the estimation of the differential cross-sections. Additional, smaller sources of error on the efficiency come from the statistics of simulated event samples and the estimated trigger efficiency.

The uncertainty on the ISR migration factors arising from misreconstruction of $\sqrt{s^{\prime}}$ has two components: an uncorrelated component from the statistics of simulated $\mathrm{e}^{+} \mathrm{e}^{-} \rightarrow \mu^{+} \mu^{-}$events; and an uncertainty estimated from switching off the simulation of the beam energy spread in the simulated sample, which was found by comparing the change in the migration factor from the standard analysis. This was determined for a single energy and applied as a correlated error over all energies. These uncertainties were estimated bin-by-bin for the differential cross-sections.

The uncertainty on the angular acceptance corrections comes from the statistics of the simulated $\mathrm{e}^{+} \mathrm{e}^{-} \rightarrow \mu^{+} \mu^{-}$samples. The uncertainty on the forward-backward asymmetry and differential cross-sections due to charge misidentification comes from the estimation of the efficiency for correctly identifying which particle had the mismeasured charge in such events. The uncertainty amounts to, at most, 0.0006 on the non-radiative forwardbackward asymmetries. This introduces an anticorrelation between bins in the forward and backward hemispheres in the differential cross-sections.

The uncertainties arising from missing higher orders in the QED radiative corrections in the simulation were estimated by taking half the difference in the measurements obtained using KK simulated events in which the highest order corrections available in the program were either included or excluded.

Uncertainties on the backgrounds come from the statistics of the simulated samples and from the number of cosmic rays failing vertex-selection cuts used to estimate the residual cosmic ray background, which are uncorrelated between energy points. The final source comes from the theoretical precision on the total cross-section of the different simulated background samples, which is correlated between energy points and is significant for the measurement of the inclusive cross-sections and forward-backward asymmetries.

Uncertainties from the evaluation of the luminosity were included in the measurements of the cross-sections. The systematic errors are summarised in Table 12.

\section{$5.3 \tau^{+} \tau^{-}$final states}

The analysis of tau pair production in 1997-98 LEP runs was presented in [4]. Here we present a complete reanalysis of those data together with the new analysis of data taken in 1999-2000. Several aspects of the analysis have changed with respect to the previous publication: improved track reconstruction algorithm, optimised event selection procedure and a better description of signal and background processes with new event generators. All the data taken in the 1997-2000 runs were analysed with a homogeneous procedure which is described below. The data taken above the $\mathrm{Z}$ resonance in the 1995-96 runs [3] were not reanalysed since the improvements of the new analysis are negligible compared to the large statistical uncertainty of those measurements.

\subsubsection{Analysis}

\section{Run selection}

Runs in which detector components essential for the analysis were not fully operational were rejected to ensure a high quality of the data used in the analysis. This included the requirement that the TPC, HPC and Forward Electromagnetic Calorimeters (FEMC) 
had a performance close to optimal. In addition, from the two pairs of detectors VD and Inner Detector (ID), and the forward tracking chambers, FCA and FCB, at least one of the two detectors in both pairs had to be operational. About $2.5 \%$ of total integrated luminosity was rejected by this procedure.

\section{Track and photon selection}

The $\tau$-pair selection was based on the reconstructed kinematic properties of the events. Therefore it was important that only well reconstructed tracks and photons were used in the analysis. To ensure this the following selection was applied to the tracks found by the reconstruction program:

- The particle momentum had to be at least $100 \mathrm{MeV} / c$;

- The error of the momentum reconstruction had to be less than the absolute value of the momentum;

- The track extrapolation had to originate from the region around the nominal interaction point (less than $5 \mathrm{~cm}$ in the plane perpendicular to the beam, less than 10 $\mathrm{cm}$ along the $\mathrm{z}$ axis);

- The track had to be seen either in VD or in ID;

- The track had to be seen in at least one tracking device beyond VD and ID (namely TPC, OD, FCA, FCB).

The last condition was not applied to the tracks close to the azimuthal boundaries of the TPC sectors, nor was it applied to the tracks pointing to the broken TPC sector for the last period of 2000 data taking. In these cases, if the last condition was not fulfilled the track was still accepted if it had a measured RICH tracking point or if there was any deposition in an electromagnetic or hadron calorimeter within 10 degrees from the track direction.

An unassociated electromagnetic cluster was accepted as a photon if it had more than $0.5 \mathrm{GeV}$ of energy deposition and was found more than one degree from the nearest track. Identified electron-positron pairs compatible with a photon conversion were considered as photons rather than charged particles.

The event selection was based on the tracks and photons accepted by these procedures.

\section{Event selection}

The selection of $\tau$-pairs was similar to the one presented in [4]. Several cuts were changed to optimise the product of efficiency and purity.

Events with fewer than 2 tracks were rejected. The charged particle tracks were grouped into two jets using the LUCLUS algorithm [20]. Tracks in each jet were considered as decay products of a $\tau$-candidate. The most energetic charged particle in each jet was called the leading charged particle and its track was called the leading track. The momentum direction of the $\tau$-candidate was approximated by the vector sum of momenta of its charged decay products.

Under the assumption that a single ISR photon is emitted along the beam direction, the $\tau$-candidate momenta can be estimated (neglecting the $\tau$ mass) from:

$$
P_{i}^{\tau}=2 \cdot \frac{\sin \theta_{j}}{\sin \theta_{1}+\sin \theta_{2}+\left|\sin \left(\theta_{1}+\theta_{2}\right)\right|} P_{\text {beam }}
$$


where $\theta_{i}$ are the polar angles of the tau-candidate momenta, and $j$ denotes the $\tau$ candidate opposite to the candidate $i$. The reduced centre-of-mass energy, $\sqrt{s^{\prime}}$, is then given by:

$$
\frac{s^{\prime}}{s}=\frac{\sin \theta_{1}+\sin \theta_{2}-\left|\sin \left(\theta_{1}+\theta_{2}\right)\right|}{\sin \theta_{1}+\sin \theta_{2}+\left|\sin \left(\theta_{1}+\theta_{2}\right)\right|} .
$$

The $\tau$ pairs were selected using the set of cuts listed below:

- To reject multi-hadronic events the number of selected tracks $N_{c h}$ had to be less than seven: $N_{c h}<7$. For events in which both jets contained more than one track the invariant mass of each jet (computed using the charged particles, which were assumed to be pions) had to be less than $2.5 \mathrm{GeV} / c^{2}$;

- The background from WW pairs, ZZ pairs and the $\mathrm{e}^{+} \mathrm{e}^{-} \rightarrow \mathrm{e}^{+} \mathrm{e}^{-\mathrm{f} \overline{\mathrm{f}}}$ process was suppressed by requiring that the acoplanarity of the two leading tracks had to be less than 12 degrees: $\theta_{A C O P}<12^{\circ}$;

- To suppress background from two-photon collisions the visible energy (computed as a scalar sum of charged particle momenta and photon energies) had to be larger than $20 \%$ of the nominal centre-of-mass energy: $\mathrm{E}_{\mathrm{VIS}}>0.2 \sqrt{\mathrm{s}}$. The cut value was increased to $0.225 \sqrt{s}$ for $\mathrm{e}^{+} \mathrm{e}^{-}$and $\mu^{+} \mu^{-}$candidates (events with exactly two tracks both satisfying loose electron identification or muon identification criteria);

- Additional suppression of two-photon background was achieved using a cut on the transverse momentum $\mathrm{P}_{T}$ (computed as the projection onto the $\mathrm{r}-\phi$ plane of the vector sum of all charged particle momenta): $\mathrm{P}_{T}<2.5 \mathrm{GeV} / c$. The cut value was increased to $8 \mathrm{GeV} / c$ for $\mathrm{e}^{+} \mathrm{e}^{-}$and $\mu^{+} \mu^{-}$candidates;

- The background from $\mathrm{e}^{+} \mathrm{e}^{-} \rightarrow \mu^{+} \mu^{-}$and partially from $\mathrm{e}^{+} \mathrm{e}^{-} \rightarrow \mathrm{e}^{+} \mathrm{e}^{-}$events was rejected using the "radial momentum" variable, $\mathrm{P}_{\mathrm{RAD}}=\sqrt{\left(\mathrm{p}_{1} / \mathrm{P}_{1}^{\tau}\right)^{2}+\left(\mathrm{p}_{2} / \mathrm{P}_{2}^{\tau}\right)^{2}}$, where $p_{i}$ are the momenta of leading charged particles and $P_{i}^{\tau}$ are the $\tau$ momenta estimated using eqn. 10. The selection cut was $\mathrm{P}_{\mathrm{RAD}}<0.95$ for $\mu^{+} \mu^{-}$candidates and $\mathrm{P}_{\mathrm{RAD}}<1.2$ for all other events;

- The bulk of the $\mathrm{e}^{+} \mathrm{e}^{-} \rightarrow \mathrm{e}^{+} \mathrm{e}^{-}$background was rejected using the depositions in the electromagnetic calorimeters HPC and FEMC. The "reduced electromagnetic energies" $e_{i}$ were defined for each jet as $e_{i}=E_{i} / E_{i}^{\tau}$, where $E_{i}$ are the total electromagnetic energy depositions in cones of half angle $30^{\circ}$ around the two leading charged particles and $E_{i}^{\tau}$ are the estimated $\tau$ energies, computed from the estimated momenta $P_{i}^{\tau}$ of eqn. 10. For the events contained within the HPC acceptance both reduced electromagnetic energies had to satisfy the condition $e_{i}<0.85$. In addition, the distance between the point with coordinates $\left(e_{1}, e_{2}\right)$ and the point $(1,1)$ expected for $\mathrm{e}^{+} \mathrm{e}^{-} \rightarrow \mathrm{e}^{+} \mathrm{e}^{-}$events had to be more than 0.5 . In the forward part of the detector the $\mathrm{e}^{+} \mathrm{e}^{-} \rightarrow \mathrm{e}^{+} \mathrm{e}^{-}$background was significantly higher than in the barrel part. Therefore the selection cuts were slightly tighter for the events contained within the FEMC acceptance and an additional cut was applied: the energy deposited beyond the first layer of the HCAL had to be non-zero;

- The background from cosmic-ray events was rejected using the impact parameters of the two leading tracks in the $\mathrm{r}-\phi$ plane $\left(R_{1,2}^{I M P}\right)$ and along the $\mathrm{z}$ axis $\left(Z_{1,2}^{I M P}\right)$. The values of $Z_{1,2}^{I M P}$ had to be not simultaneously larger than $+3 \mathrm{~cm}$ or simultaneously smaller than $-3 \mathrm{~cm}$ and at least one of $R_{1}^{I M P}$ or $R_{2}^{I M P}$ had to be less than $3 \mathrm{~mm}$ in absolute value. In addition, for the two-track events, the difference $\left|Z_{1}^{I M P}-Z_{2}^{I M P}\right|$ had to be less than $3 \mathrm{~cm}$;

- The remaining background from $\mathrm{e}^{+} \mathrm{e}^{-} \rightarrow \mathrm{e}^{+} \mathrm{e}^{-}, \mathrm{e}^{+} \mathrm{e}^{-} \rightarrow \mu^{+} \mu^{-}$and cosmic-ray events was reduced by rejecting back-to-back events with very low acollinearity between the two leading tracks: $\theta_{A C O L}<0.3^{\circ}$. 


\section{DELPHI}

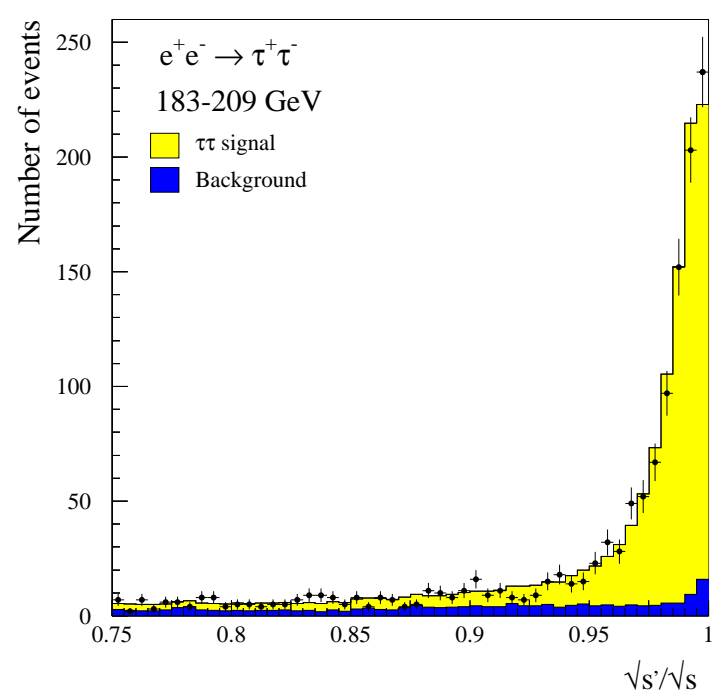

DELPHI

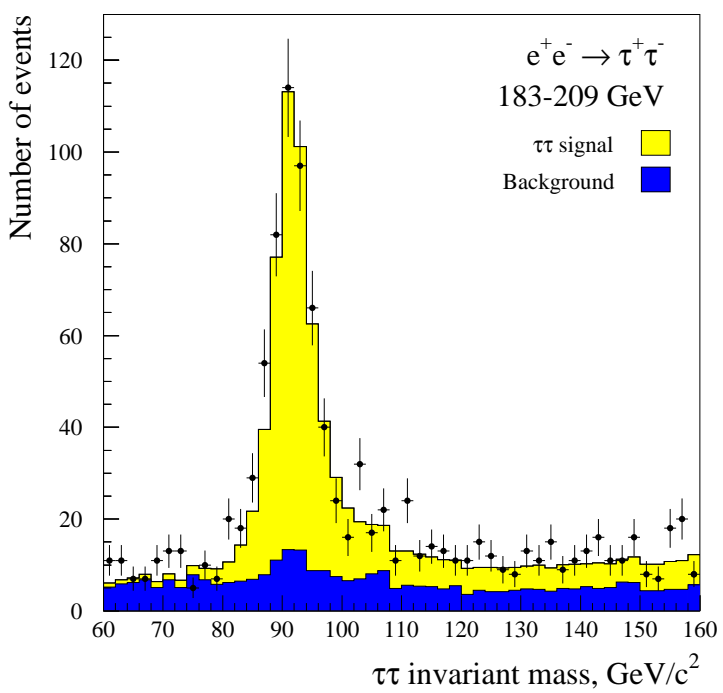

Figure 5: The reconstructed $\sqrt{s^{\prime}} / \sqrt{s}$ distribution (left) and the reconstructed $\sqrt{s^{\prime}}$ distribution (right) for $\mathrm{e}^{+} \mathrm{e}^{-} \rightarrow \tau^{+} \tau^{-}$events. Both plots show data from 1997-2000.

After the selection procedure the residual background level was relatively low (10$20 \%)$ for non-radiative events and for radiative return events. However the region of intermediate values of reconstructed $\sqrt{s^{\prime}}$ was dominated by background from $\gamma \gamma \rightarrow \mathrm{e}^{+} \mathrm{e}^{-}$ events. Therefore all $\mathrm{e}^{+} \mathrm{e}^{-}$candidate events were rejected in the region $\sqrt{1 / 3}<\sqrt{s^{\prime}} / \sqrt{s}<$ 0.85 .

Events were associated to the non-radiative or inclusive samples according to the value of $\sqrt{s^{\prime}}$ estimated using eqn. 11. The distribution of the reduced centre-of-mass energy, for selected $\tau$ pair events from centre-of-mass energies of 183 to $207 \mathrm{GeV}$, is shown in Figure 5. The ratio $\sqrt{s^{\prime}} / \sqrt{s}$ is shown for the non-radiative sample, while for the events with a hard ISR photon the value of $\sqrt{s^{\prime}}$, which approximates the invariant mass of the final state leptons, is shown.

\section{Estimation of the selection efficiency and residual background}

Unlike in the cases of $\mathrm{e}^{+} \mathrm{e}^{-} \rightarrow \mathrm{e}^{+} \mathrm{e}^{-}$and $\mathrm{e}^{+} \mathrm{e}^{-} \rightarrow \mu^{+} \mu^{-}$, the selection efficiency of $\mathrm{e}^{+} \mathrm{e}^{-} \rightarrow \tau^{+} \tau^{-}$events cannot be estimated or verified from real data. The estimation of the selection efficiency is entirely based on simulations. To ensure good agreement between real and simulated data an extensive study was performed using test samples of real and simulated events. Where necessary, the simulation was corrected by introducing calibration constants, smearing distributions, etc.

The ( $\theta$-dependent) energy scale and energy resolution of the electromagnetic calorimeters were calibrated using well reconstructed $\mathrm{e}^{+} \mathrm{e}^{-} \rightarrow \mathrm{e}^{+} \mathrm{e}^{-}$events selected from high energy runs. Non-linearities were checked using $\mathrm{e}^{+} \mathrm{e}^{-} \rightarrow \mathrm{e}^{+} \mathrm{e}^{-}$events selected from Z runs and $\gamma \gamma \rightarrow \mathrm{e}^{+} \mathrm{e}^{-}$events at high energies. The corresponding re-scaling and smearing were applied to electromagnetic energies in simulated events. Also, a small forward-backward asymmetry of the electromagnetic calorimeters was found and corrected for. 
The absolute scale and resolution of charged-particle momentum measurements were calibrated using $\mathrm{e}^{+} \mathrm{e}^{-} \rightarrow \mu^{+} \mu^{-}$events from $\mathrm{Z}$ runs. The real data needed a small $\theta$ dependent correction to the measured $1 / p_{T}$, while simulated data needed a smearing of the $1 / p_{T}$ distribution. The effect of this calibration was checked with non-radiative $\mathrm{e}^{+} \mathrm{e}^{-} \rightarrow \mu^{+} \mu^{-}$events from high energy data. A rather small (about $2 \sigma$ ) discrepancy was found and taken as a systematic uncertainty.

The momentum dependence of the muon chamber efficiency was studied using test samples of muons selected with very tight HCAL criteria. The $\mathrm{e}^{+} \mathrm{e}^{-} \rightarrow \mu^{+} \mu^{-}$events from $\mathrm{Z}$ and high energy runs were used to select muons with $\sim 45 \mathrm{GeV} / c$ and $\sim 100$ $\mathrm{GeV} / c$ momenta, while $\gamma \gamma \rightarrow \mu^{+} \mu^{-}$and $\tau \rightarrow \mu \nu \nu$ events from high energy runs were used for momentum regions of $0-45$ and $45-100 \mathrm{GeV} / c$. Both the mean efficiency and momentum dependence were corrected in the simulation.

Other calibrations included the electron punch-through in the first layer of the HCAL, the specific energy loss $(\mathrm{dE} / \mathrm{dx})$ measurements from the TPC and the LEP energy spread. After applying all necessary corrections and calibrations the selection efficiency was calculated from simulated data as the fraction of events generated above the nominal $\sqrt{s^{\prime}}$ cut which pass the selection criteria.

The residual background level was normalised using the real data. For each type of background a selection cut most sensitive to this background source was chosen and all other cuts were applied to the real and simulated data. The events failing the "sensitive cut" were then used to normalise the background level predicted by simulation to the real data. As an example the distributions of acoplanarity (sensitive to four-fermion background) and radial momentum (sensitive to $\mathrm{e}^{+} \mathrm{e}^{-} \rightarrow \mu^{+} \mu^{-}$) are presented in Figure 6 . The full statistics of the 1997-2000 runs are shown. Arrows show the selection cuts; events to the left of the arrows were selected as $\tau$ candidates and events to the right were used for background level normalisation.

Due to this normalisation procedure the simulation is used only to predict the shapes of distributions, while the absolute background level is estimated from the real data. The size of the corrections applied to the simulation was of the order of $5-15 \%$. The estimated residual backgrounds from different channels are presented in Table 13 for non-radiative and inclusive samples selected from 2000 data. For other years the background levels were similar. The estimation of background uncertainties is discussed in section 5.3.2.

The feed-through of $\mathrm{e}^{+} \mathrm{e}^{-} \rightarrow \tau^{+} \tau^{-}$events from lower values of $\sqrt{s^{\prime}} / \sqrt{s}$ into the nonradiative sample was estimated from simulated events to be approximately $4.4 \%$, almost independent of $\sqrt{s}$.

\subsubsection{Results}

\section{Cross-sections}

The total cross-sections of $\tau$ pair production for the non-radiative and inclusive samples were calculated as follows:

$$
\sigma_{\tau \tau}=\frac{\left(N_{s e l}-N_{b g}\right)(1-f)}{\epsilon \mathcal{L}} .
$$

Here $\mathcal{L}$ is the total integrated luminosity for the corresponding energy point, $N_{\text {sel }}$ is the number of events selected in the corresponding sample, $f$ (applicable to the nonradiative cross-section only) is the fraction of feed-through events estimated from the $\tau$ pair simulation and $\epsilon$ is the selection efficiency, computed within the full $4 \pi$ acceptance, 
DELPHI

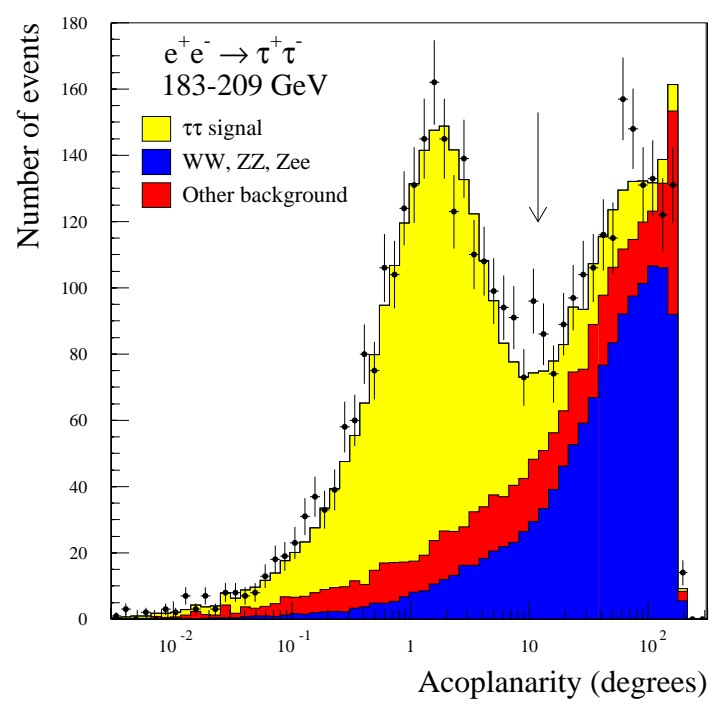

DELPHI

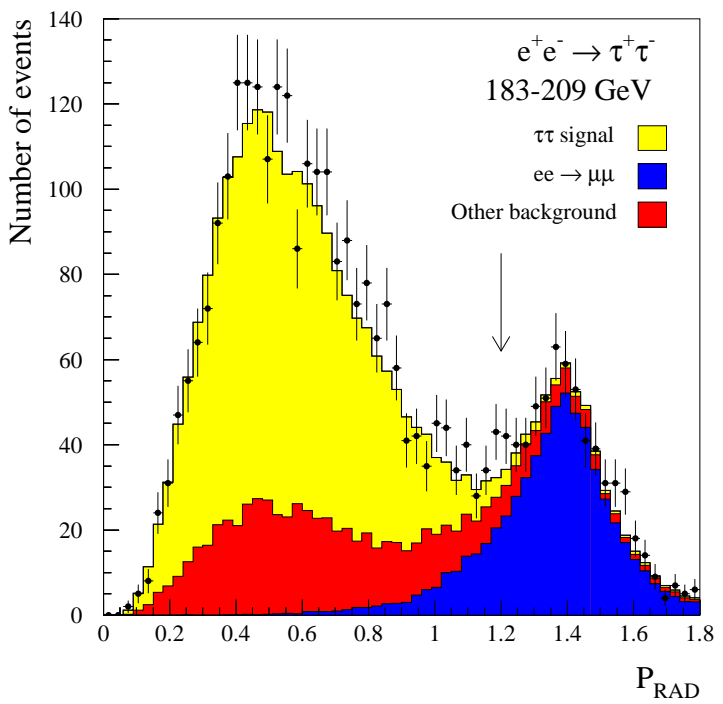

Figure 6: The acoplanarity distribution (left) and the distribution of the variable $\mathrm{P}_{\mathrm{RAD}}$ (right). The acoplanarity distribution is sensitive to the four-fermion backgrounds while the $\mathrm{P}_{\mathrm{RAD}}$ distribution is sensitive to the $\mathrm{e}^{+} \mathrm{e}^{-} \rightarrow \mu^{+} \mu^{-}$background. Both plots show data from 1997-2000. The dominant backgrounds have been normalised to obtain good agreement between simulation and data in the region dominated by background.

\begin{tabular}{|l|c|c|}
\hline \multicolumn{3}{|c|}{$\mathrm{e}^{+} \mathrm{e}^{-} \rightarrow \tau^{+} \tau^{-}$} \\
\hline \hline $\begin{array}{l}\text { Background } \\
\text { source }\end{array}$ & $\begin{array}{c}\text { Non-radiative } \\
\text { background }\end{array}$ & $\begin{array}{c}\text { Inclusive } \\
\text { background }\end{array}$ \\
\hline \hline $\mathrm{e}^{+} \mathrm{e}^{-} \rightarrow \mathrm{e}^{+} \mathrm{e}^{-}$ & $4.50 \pm 0.54$ & $3.37 \pm 0.37$ \\
$\mathrm{e}^{+} \mathrm{e}^{-} \rightarrow \mu^{+} \mu^{-}$ & $1.05 \pm 0.15$ & $1.18 \pm 0.16$ \\
$\mathrm{e}^{+} \mathrm{e}^{-} \rightarrow \mathrm{q} \bar{q}$ & $0.19 \pm 0.03$ & $1.40 \pm 0.14$ \\
$\gamma \gamma \rightarrow \tau^{+} \tau^{-}$ & $0.02 \pm 0.01$ & $0.23 \pm 0.05$ \\
$\gamma \gamma \rightarrow \mathrm{e}^{+} \mathrm{e}^{-}$ & $0.11 \pm 0.05$ & $1.89 \pm 0.31$ \\
$\gamma \gamma \rightarrow \mu^{+} \mu^{-}$ & $0.07 \pm 0.04$ & $0.23 \pm 0.06$ \\
$\gamma \gamma \rightarrow \mathrm{q} \overline{\mathrm{q}}$ & $0.00 \pm 0.04$ & $0.10 \pm 0.06$ \\
$\mathrm{e}^{+} \mathrm{e}^{-} \rightarrow \mathrm{W}^{+} \mathrm{W}^{-}$ & $2.93 \pm 0.50$ & $3.15 \pm 0.54$ \\
$\mathrm{e}^{+} \mathrm{e}^{-} \rightarrow \mathrm{ZZ}$ & $1.09 \pm 0.19$ & $2.04 \pm 0.35$ \\
$\mathrm{e}^{+} \mathrm{e}^{-} \rightarrow \mathrm{e}^{+} \mathrm{e}^{-} \mathrm{f} \overline{\mathrm{f}}$ & $2.19 \pm 0.38$ & $5.50 \pm 0.94$ \\
Cosmic rays & $0.28 \pm 0.22$ & $0.24 \pm 0.15$ \\
\hline \hline Total & $12.43 \pm 0.89$ & $19.34 \pm 1.26$ \\
\hline
\end{tabular}

Table 13: Residual background level for 2000 data (in \% relative to the number of selected $\tau$-pair candidates). 


\begin{tabular}{|c|c|c|c|c|c|c|c|c|}
\hline \multicolumn{9}{|c|}{$\mathrm{e}^{+} \mathrm{e}^{-} \rightarrow \tau^{+} \tau^{-}$} \\
\hline & \multicolumn{8}{|c|}{ Energy point $(\mathrm{GeV})$} \\
\hline & 183 & 189 & 192 & 196 & 200 & 202 & 205 & 207 \\
\hline Energy $(\mathrm{GeV})$ & 182.66 & 188.63 & 191.58 & 195.51 & 199.51 & 201.64 & 204.89 & 206.56 \\
\hline $\operatorname{Lumi}\left(\mathrm{pb}^{-1}\right)$ & 53.11 & 152.67 & 25.14 & 75.99 & 82.65 & 40.40 & 81.49 & 136.39 \\
\hline No. Events & 228 & 551 & 79 & 232 & 269 & 119 & 273 & 401 \\
\hline Efficiency (\%) & 52.1 & 52.2 & 52.0 & 52.0 & 52.0 & 52.0 & 51.7 & 51.7 \\
\hline Background (\%) & 12.2 & 11.8 & 14.6 & 12.6 & 13.6 & 13.8 & 12.3 & 12.5 \\
\hline
\end{tabular}

Table 14: Details of LEP II analysis for $\mathrm{e}^{+} \mathrm{e}^{-} \rightarrow \tau^{+} \tau^{-}$channel. The table shows the actual centre-of-mass energy and luminosity analysed at each energy point, the number of events selected in the inclusive analysis and the efficiencies for selecting events in the non-radiative samples and the backgrounds selected in the non-radiative samples.

\begin{tabular}{|c|c|c|c|c|}
\hline \multicolumn{5}{|c|}{$\mathrm{e}^{+} \mathrm{e}^{-} \rightarrow \tau^{+} \tau^{-}$} \\
\hline \multirow{2}{*}{$\begin{array}{c}\sqrt{s} \\
(\mathrm{GeV})\end{array}$} & \multicolumn{2}{|c|}{$\sqrt{s^{\prime}}>75(\mathrm{GeV})$} & \multicolumn{2}{|c|}{$\sqrt{s^{\prime}} / \sqrt{s}>0.85$} \\
\hline & $\sigma_{\tau \tau}(\mathrm{pb})$ & $\mathrm{A}_{\mathrm{FB}}^{\tau}$ & $\sigma_{\tau \tau}(\mathrm{pb})$ & $\mathrm{A}_{\mathrm{FB}}^{\tau}$ \\
\hline 130 & $\begin{array}{c}22.20 \pm 4.60 \pm 1.56 \\
(20.30)\end{array}$ & $\begin{array}{c}0.310 \pm 0.170 \pm 0.020 \\
(0.337)\end{array}$ & $\begin{array}{c}10.20 \pm 3.10 \pm 0.72 \\
(8.31)\end{array}$ & $\begin{array}{c}0.730 \pm 0.170 \pm 0.020 \\
(0.719)\end{array}$ \\
\hline 136 & $\begin{array}{c}17.70 \pm 3.90 \pm 1.24 \\
(17.29)\end{array}$ & $\begin{array}{c}0.260 \pm 0.190 \pm 0.020 \\
(0.338)\end{array}$ & $\begin{array}{c}8.80 \pm 3.00 \pm 0.62 \\
(7.17)\end{array}$ & $\begin{array}{c}0.490 \pm 0.230 \pm 0.020 \\
(0.699)\end{array}$ \\
\hline 161 & $\begin{array}{c}11.70 \pm 1.80 \pm 0.82 \\
(10.44)\end{array}$ & $\begin{array}{c}0.390 \pm 0.120 \pm 0.020 \\
(0.332)\end{array}$ & $\begin{array}{c}5.10 \pm 1.20 \pm 0.36 \\
(4.54)\end{array}$ & $\begin{array}{c}0.920 \pm 0.080 \pm 0.020 \\
(0.628)\end{array}$ \\
\hline 172 & $\begin{array}{c}11.20 \pm 1.80 \pm 0.79 \\
(8.83)\end{array}$ & $\begin{array}{c}0.190 \pm 0.140 \pm 0.020 \\
(0.329)\end{array}$ & $\begin{array}{c}4.50 \pm 1.10 \pm 0.32 \\
(3.89)\end{array}$ & $\begin{array}{c}0.130 \pm 0.200 \pm 0.020 \\
(0.610)\end{array}$ \\
\hline 183 & $\begin{array}{c}8.73 \pm 0.70 \pm 0.20 \\
(7.64)\end{array}$ & $\begin{array}{c}0.400 \pm 0.074 \pm 0.012 \\
(0.326)\end{array}$ & $\begin{array}{c}3.29 \pm 0.38 \pm 0.07 \\
(3.39)\end{array}$ & $\begin{array}{c}0.671 \pm 0.080 \pm 0.012 \\
(0.596)\end{array}$ \\
\hline 189 & $\begin{array}{c}7.23 \pm 0.38 \pm 0.17 \\
(7.08)\end{array}$ & $\begin{array}{c}0.448 \pm 0.047 \pm 0.012 \\
(0.324)\end{array}$ & $\begin{array}{c}3.11 \pm 0.22 \pm 0.07 \\
(3.15)\end{array}$ & $\begin{array}{c}0.697 \pm 0.048 \pm 0.011 \\
(0.589)\end{array}$ \\
\hline 192 & $\begin{array}{c}6.16 \pm 0.89 \pm 0.15 \\
(6.83)\end{array}$ & $\begin{array}{c}0.415 \pm 0.134 \pm 0.012 \\
(0.324)\end{array}$ & $\begin{array}{c}2.50 \pm 0.48 \pm 0.06 \\
(3.04)\end{array}$ & $\begin{array}{c}0.578 \pm 0.150 \pm 0.011 \\
(0.586)\end{array}$ \\
\hline 196 & $\begin{array}{c}5.97 \pm 0.51 \pm 0.14 \\
(6.52)\end{array}$ & $\begin{array}{c}0.156 \pm 0.080 \pm 0.012 \\
(0.323)\end{array}$ & $\begin{array}{c}2.89 \pm 0.30 \pm 0.06 \\
(2.91)\end{array}$ & $\begin{array}{c}0.465 \pm 0.083 \pm 0.011 \\
(0.582)\end{array}$ \\
\hline 200 & $\begin{array}{c}6.50 \pm 0.50 \pm 0.15 \\
(6.22)\end{array}$ & $\begin{array}{c}0.226 \pm 0.073 \pm 0.012 \\
(0.322)\end{array}$ & $\begin{array}{c}2.61 \pm 0.27 \pm 0.06 \\
(2.78)\end{array}$ & $\begin{array}{c}0.540 \pm 0.080 \pm 0.011 \\
(0.578)\end{array}$ \\
\hline 202 & $\begin{array}{c}5.74 \pm 0.68 \pm 0.14 \\
(6.08)\end{array}$ & $\begin{array}{c}0.314 \pm 0.110 \pm 0.012 \\
(0.321)\end{array}$ & $\begin{array}{c}2.55 \pm 0.38 \pm 0.06 \\
(2.72)\end{array}$ & $\begin{array}{c}0.464 \pm 0.122 \pm 0.011 \\
(0.576)\end{array}$ \\
\hline 205 & $\begin{array}{c}6.94 \pm 0.52 \pm 0.16 \\
(5.86)\end{array}$ & $\begin{array}{c}0.434 \pm 0.070 \pm 0.012 \\
(0.320)\end{array}$ & $\begin{array}{c}2.80 \pm 0.28 \pm 0.06 \\
(2.62)\end{array}$ & $\begin{array}{c}0.709 \pm 0.068 \pm 0.011 \\
(0.574)\end{array}$ \\
\hline 207 & $\begin{array}{c}5.95 \pm 0.38 \pm 0.14 \\
(5.76)\end{array}$ & $\begin{array}{c}0.347 \pm 0.060 \pm 0.012 \\
(0.320)\end{array}$ & $\begin{array}{c}2.53 \pm 0.21 \pm 0.06 \\
(2.58)\end{array}$ & $\begin{array}{c}0.666 \pm 0.059 \pm 0.011 \\
(0.572)\end{array}$ \\
\hline
\end{tabular}

Table 15: Measured cross-sections and forward-backward asymmetries for inclusive and non-radiative $\mathrm{e}^{+} \mathrm{e}^{-} \rightarrow \tau^{+} \tau^{-}$events. The first uncertainty is statistical, the second systematic. Numbers in brackets are the theoretical predictions of ZFITTER, which are estimated to have a precision of $\pm 0.4 \%$ on $\sigma_{\tau \tau}$ and \pm 0.004 on $\mathrm{A}_{\mathrm{FB}}^{\tau}$. 
which takes into account the effect of selection cuts (including the cut on $\sqrt{s^{\prime}}$ ) and the trigger efficiency. The trigger efficiency was estimated from the real data using the redundancy of the DELPHI trigger system. Its typical value was about $99.9 \%$ with typical uncertainty of about $0.05 \%$. For the 2000 data taken with one TPC sector broken the trigger efficiency value was $98.9 \pm 0.9 \%$ (for the whole detector). The values of the selection efficiency are summarised in Table 14. The residual number of background events $N_{b g}$ was estimated from simulation and normalised to the real data as discussed in the previous section. The measured cross-sections are presented in Table 15 and shown in Figure 10.

\section{Forward-backward asymmetries}

The forward-backward asymmetry of $\tau$ pair production was calculated as

$$
A_{F B}^{\tau \tau}=\frac{\sigma_{F}-\sigma_{B}}{\sigma_{F}+\sigma_{B}}
$$

The forward cross-section $\sigma_{F}\left(0^{\circ}<\theta_{\tau^{-}}<90^{\circ}\right)$ and the backward cross-section $\sigma_{B}$ $\left(90^{\circ}<\theta_{\tau^{-}}<180^{\circ}\right)$ were determined in a similar way to the determination of the total cross-section $\sigma_{\tau \tau}$. The $\tau$ charge and the direction of the $\tau$ momentum were estimated from the charges and momenta of its charged decay products. To reduce charge misidentification, only events with at least one jet consisting of exactly one charged particle (1-N topology events) were used in this analysis. The charge of that single particle was assigned to the corresponding $\tau$ and an opposite charge was assigned to the other $\tau$ regardless of the charge of the multi-track jet. The 1-1 topology events with two reconstructed tracks of the same charge (like-sign events) were excluded from the analysis. For the period of broken TPC sector in 2000 the tracks detected only in the VD and ID were not used for the charge identification.

Misidentification of the $\tau$ charge results in a reduction of the absolute value of $A_{F B}$. The probability of charge misidentification was estimated from the real data using the rate of the like-sign events. The corresponding correction to $A_{F B}$ was found to be $+0.0068 \pm$ 0.0034 (accidentally the same for non-radiative and inclusive samples). The corrected values of $A_{F B}$ are summarised in Table 15 and shown in Figure 11.

\section{Differential cross-sections}

In addition to the measurements of the forward-backward asymmetries, differential cross-sections, $\mathrm{d} \sigma / \mathrm{d} \cos \theta$ for $\tau$-pair production were determined. The region of angular acceptance of this analysis $(-0.96<\cos \theta<0.96)$ was divided into ten bins with respect to the polar angle of the momentum direction of the negatively charged tau lepton. The cross-section for every bin was determined similarly to the determination of the total cross-section and the correction for the charge misidentification effect was applied. The measured differential cross-sections are presented in Table 16.

\section{Systematic errors}

A breakdown of the systematic uncertainties for cross-sections and asymmetries of non-radiative and inclusive samples is presented in Table 17. The numbers are given for the results of 2000; for other years the uncertainties were of a similar size.

The most important sources of systematic uncertainty were the choice of track- and event-selection cuts, background level and detector calibration. To estimate the effect of 


\begin{tabular}{|c|c|c|c|c|c|c|c|c|}
\hline \multicolumn{9}{|c|}{$\mathrm{e}^{+} \mathrm{e}^{-} \rightarrow \tau^{+} \tau^{-}$} \\
\hline \multicolumn{3}{|c|}{$\sqrt{s} \sim 183$} & \multicolumn{3}{|c|}{$\sqrt{s} \sim 189$} & \multicolumn{3}{|c|}{$\sqrt{s} \sim 192$} \\
\hline \multirow[b]{2}{*}{$\cos \theta$} & \multicolumn{2}{|r|}{$\mathrm{d} \sigma / \mathrm{d} \cos \theta(\mathrm{pb})$} & \multirow[b]{2}{*}{$\cos \theta$} & \multicolumn{2}{|r|}{$\mathrm{d} \sigma / \mathrm{d} \cos \theta(\mathrm{pb})$} & \multirow[b]{2}{*}{$\cos \theta$} & \multicolumn{2}{|r|}{$\mathrm{d} \sigma / \mathrm{d} \cos \theta(\mathrm{pb})$} \\
\hline & SM & Measurement & & SM & Measurement & & SM & Measurement \\
\hline$[-0.96,-0.80]$ & 0.48 & $-0.17 \pm 0.00(0.50) \pm 0.03$ & 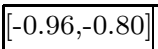 & 0.47 & $\overline{0.19 \pm 0.20(0.27) \pm 0.03}$ & {$[-0.96,-0.80]$} & 0.46 & $0.60 \pm 0.74(0.69) \pm 0.02$ \\
\hline$[-0.80,-0.60]$ & 0.49 & $0.54 \pm 0.37(0.36) \pm 0.03$ & {$[-0.80,-0.60]$} & 0.47 & $0.13 \pm 0.14(0.21) \pm 0.03$ & {$[-0.80,-0.60]$} & 0.46 & $0.22 \pm 0.41(0.52) \pm 0.03$ \\
\hline$[-0.60,-0.40]$ & 0.59 & $0.42 \pm 0.28(0.32) \pm 0.02$ & {$[-0.60,-0.40]$} & 0.56 & $0.35 \pm 0.16(0.18) \pm 0.03$ & {$[-0.60,-0.40]$} & 0.54 & $0.45 \pm 0.41(0.45) \pm 0.01$ \\
\hline$[-0.40,-0.20]$ & 0.78 & $0.44 \pm 0.28(0.36) \pm 0.02$ & {$[-0.40,-0.20]$} & 0.73 & $0.74 \pm 0.21(0.21) \pm 0.03$ & {$[-0.40,-0.20]$} & 0.71 & $0.44 \pm 0.41(0.50) \pm 0.02$ \\
\hline$[-0.20,0.00]$ & 1.08 & $1.22 \pm 0.48(0.46) \pm 0.04$ & {$[-0.20,0.00]$} & 1.00 & $0.78 \pm 0.24(0.26) \pm 0.04$ & {$[-0.20,0.00]$} & 0.97 & $0.85 \pm 0.60(0.63) \pm 0.03$ \\
\hline$[0.00,0.20]$ & 1.47 & $1.51 \pm 0.53(0.52) \pm 0.05$ & {$[0.00,0.20]$} & 1.36 & $1.78 \pm 0.34(0.30) \pm 0.04$ & {$[0.00,0.20]$} & 1.32 & $1.48 \pm 0.76(0.72) \pm 0.03$ \\
\hline $0.20,0.40]$ & 1.97 & $1.86 \pm 0.55(0.55) \pm 0.06$ & $0.20,0.40]$ & 1.83 & $2.31 \pm 0.35(0.31) \pm 0.05$ & $0.20,0.40]$ & 1.76 & $1.77 \pm 0.77(0.77) \pm 0.04$ \\
\hline $0.40,0.60]$ & 2.58 & $2.30 \pm 0.61(0.64) \pm 0.07$ & $0.40,0.60]$ & 2.39 & $2.59 \pm 0.37(0.36) \pm 0.06$ & $0.40,0.60]$ & 2.30 & $1.67 \pm 0.77(0.88) \pm 0.05$ \\
\hline $0.60,0.80]$ & 3.30 & $3.76 \pm 0.94(0.89) \pm 0.12$ & $0.60,0.80]$ & 3.06 & $2.61 \pm 0.48(0.51) \pm 0.11$ & $0.60,0.80]$ & 2.95 & $2.57 \pm 1.18(1.25) \pm 0.07$ \\
\hline $0.80,0.96]$ & 4.08 & $4.77 \pm 1.40(1.31) \pm 0.15$ & $0.80,0.96]$ & 3.78 & $3.14 \pm 0.67(0.73) \pm 0.13$ & $0.80,0.96$ & 3.65 & $0.25 \pm 0.74(1.75) \pm 0.09$ \\
\hline \multicolumn{3}{|c|}{$\sqrt{s} \sim 196$} & \multicolumn{3}{|c|}{$\sqrt{s} \sim 200$} & \multicolumn{3}{|c|}{$\sqrt{s} \sim 202$} \\
\hline \multirow[b]{2}{*}{$\cos \theta$} & \multicolumn{2}{|r|}{$\mathrm{d} \sigma / \mathrm{d} \cos \theta(\mathrm{pb})$} & \multirow[b]{2}{*}{$\cos \theta$} & \multicolumn{2}{|r|}{$\mathrm{d} \sigma / \mathrm{d} \cos \theta(\mathrm{pb})$} & \multirow[b]{2}{*}{$\cos \theta$} & \multicolumn{2}{|r|}{$\mathrm{d} \sigma / \mathrm{d} \cos \theta(\mathrm{pb})$} \\
\hline & SM & Measurement & & $\mathrm{SM}$ & Measurement & & $\mathrm{SM}$ & Measurement \\
\hline 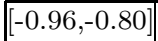 & 0.45 & $0.35 \pm 0.34(0.39) \pm 0.01$ & 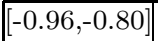 & 0.44 & $0.32 \pm 0.32(0.36) \pm 0.01$ & {$[-0.96,-0.80]$} & 0.44 & $-0.13 \pm 0.00(0.52) \pm 0.01$ \\
\hline$[-0.80,-0.60]$ & 0.45 & $0.36 \pm 0.27(0.29) \pm 0.03$ & {$[-0.80,-0.60]$} & 0.44 & $0.81 \pm 0.35(0.28) \pm 0.03$ & {$[-0.80,-0.60]$} & 0.43 & $0.83 \pm 0.50(0.39) \pm 0.03$ \\
\hline$[-0.60,-0.40]$ & 0.52 & $1.02 \pm 0.33(0.25) \pm 0.01$ & {$[-0.60,-0.40]$} & 0.51 & $0.50 \pm 0.23(0.24) \pm 0.01$ & {$[-0.60,-0.40]$} & 0.50 & $0.42 \pm 0.31(0.34) \pm 0.01$ \\
\hline$[-0.40,-0.20]$ & 0.68 & $0.73 \pm 0.29(0.28) \pm 0.02$ & {$[-0.40,-0.20]$} & 0.65 & $0.49 \pm 0.24(0.27) \pm 0.02$ & {$[-0.40,-0.20]$} & 0.64 & $0.60 \pm 0.36(0.38) \pm 0.02$ \\
\hline$[-0.20,0.00]$ & 0.92 & $1.31 \pm 0.41(0.35) \pm 0.03$ & {$[-0.20,0.00]$} & 0.88 & $0.98 \pm 0.35(0.33) \pm 0.03$ & {$[-0.20,0.00]$} & 0.86 & $1.54 \pm 0.61(0.47) \pm 0.03$ \\
\hline$[0.00,0.20]$ & 1.25 & $1.03 \pm 0.37(0.40) \pm 0.03$ & {$[0.00,0.20]$} & 1.20 & $0.93 \pm 0.34(0.38) \pm 0.03$ & {$[0.00,0.20]$} & 1.17 & $0.65 \pm 0.42(0.54) \pm 0.03$ \\
\hline$[0.20,0.40]$ & 1.68 & $1.38 \pm 0.40(0.43) \pm 0.04$ & $0.20,0.40]$ & 1.60 & $1.96 \pm 0.44(0.41) \pm 0.04$ & $0.20,0.40]$ & 1.56 & $2.46 \pm 0.70(0.58) \pm 0.03$ \\
\hline$[0.40,0.60]$ & 2.19 & $2.24 \pm 0.50(0.50) \pm 0.05$ & $0.40,0.60]$ & 2.09 & $1.86 \pm 0.44(0.47) \pm 0.05$ & $0.40,0.60]$ & 2.04 & $2.01 \pm 0.65(0.66) \pm 0.05$ \\
\hline$[0.60,0.80]$ & 2.81 & $2.85 \pm 0.71(0.70) \pm 0.07$ & $0.60,0.80]$ & 2.68 & $2.20 \pm 0.61(0.66) \pm 0.07$ & $0.60,0.80]$ & 2.62 & $1.11 \pm 0.69(0.93) \pm 0.07$ \\
\hline $0.80,0.96]$ & 3.48 & $2.92 \pm 0.91(0.98) \pm 0.08$ & {$[0.80,0.96]$} & 3.32 & $2.89 \pm 0.87(0.92) \pm 0.08$ & $0.80,0.96]$ & 3.24 & $2.28 \pm 1.12(1.30) \pm 0.08$ \\
\hline \multicolumn{3}{|c|}{$\sqrt{s} \sim 205$} & & & & \multicolumn{3}{|c|}{$\sqrt{s} \sim 207$} \\
\hline \multirow[b]{2}{*}{$\cos \theta$} & \multicolumn{2}{|r|}{ ( $\mathrm{d} \sigma / \mathrm{d} \cos \theta(\mathrm{pb})$} & & & & \multirow[b]{2}{*}{$\cos \theta$} & \multicolumn{2}{|r|}{$\mathrm{d} \sigma / \mathrm{d} \cos \theta(\mathrm{pb})$} \\
\hline & SM & Measurement & & & & & $\mathrm{SM}$ & Measurement \\
\hline$[-0.96,-0.80]$ & 0.43 & $-0.12 \pm 0.00(0.36) \pm 0.01$ & & & & {$[-0.96,-0.80]$} & 0.43 & $0.30 \pm 0.23(0.27) \pm 0.01$ \\
\hline$[-0.80,-0.60]$ & 0.42 & $0.48 \pm 0.23(0.27) \pm 0.02$ & & & & {$[-0.80,-0.60]$} & 0.42 & $0.00 \pm 0.15(0.21) \pm 0.02$ \\
\hline$[-0.60,-0.40]$ & 0.48 & $0.44 \pm 0.23(0.23) \pm 0.01$ & & & & {$[-0.60,-0.40]$} & 0.48 & $0.50 \pm 0.18(0.18) \pm 0.01$ \\
\hline$[-0.40,-0.20]$ & 0.62 & $0.53 \pm 0.26(0.26) \pm 0.01$ & & & & {$[-0.40,-0.20]$} & 0.61 & $0.60 \pm 0.20(0.20) \pm 0.01$ \\
\hline$[-0.20,0.00]$ & 0.83 & $0.48 \pm 0.28(0.33) \pm 0.02$ & & & & {$[-0.20,0.00]$} & 0.82 & $0.62 \pm 0.22(0.25) \pm 0.02$ \\
\hline$[0.00,0.20]$ & 1.13 & $1.29 \pm 0.39(0.37) \pm 0.02$ & & & & {$[0.00,0.20]$} & 1.11 & $1.66 \pm 0.32(0.28) \pm 0.02$ \\
\hline$[0.20,0.40]$ & 1.50 & $1.30 \pm 0.37(0.40) \pm 0.03$ & & & & $0.20,0.40]$ & 1.48 & $1.61 \pm 0.32(0.31) \pm 0.03$ \\
\hline$[0.40,0.60]$ & 1.97 & $2.25 \pm 0.49(0.46) \pm 0.04$ & & & & $0.40,0.60]$ & 1.93 & $1.54 \pm 0.32(0.35) \pm 0.04$ \\
\hline$[0.60,0.80]$ & 2.52 & $2.87 \pm 0.66(0.64) \pm 0.06$ & & & & $0.60,0.80]$ & 2.48 & $1.72 \pm 0.42(0.49) \pm 0.06$ \\
\hline$[0.80,0.96]$ & 3.13 & $4.99 \pm 1.08(0.90) \pm 0.07$ & & & & $0.80,0.96$ & 3.07 & $4.32 \pm 0.81(0.69) \pm 0.07$ \\
\hline
\end{tabular}

Table 16: Differential cross-sections for non-radiative $\mathrm{e}^{+} \mathrm{e}^{-} \rightarrow \tau^{+} \tau^{-}$events at centre-of-mass energies from 183 to $20^{\prime} 7 \mathrm{GeV}$. The tables show the bins, the predictions of the Standard Model and the measurements. The errors quoted are the statistical and experimental systematic errors. The statistical errors are shown as the measured errors and, in brackets, the expected errors, computed from the square root of the observed and expected numbers of events respectively. 


\begin{tabular}{|l|c|c||c|c|}
\hline \multicolumn{5}{|c|}{$\mathrm{e}^{+} \mathrm{e}^{-} \rightarrow \tau^{+} \tau^{-}$} \\
\hline \hline Source & $\Delta \sigma / \sigma$ & $\Delta \sigma / \sigma$ & $\Delta A_{F B}$ & $\Delta A_{F B}$ \\
(non-rad.) & (inclus.) & (non-rad.) & (inclus.) \\
\hline \hline Track Selection & 72 & 35 & 34 & 48 \\
Event Selection & 127 & 104 & 51 & 59 \\
Detector Calibration & 91 & 86 & 58 & 50 \\
Background Level & 102 & 157 & 39 & 43 \\
Light pair subtraction & 9 & 12 & 4 & 3 \\
Trigger Efficiency & 9 & 9 & 0 & 0 \\
$\sqrt{s^{\prime}}$ Reconstruction & 25 & 3 & 14 & 1 \\
Feed-through & 32 & 2 & 17 & 1 \\
Charge Misidentification & 0 & 0 & 34 & 34 \\
Simulation Statistics & 21 & 19 & 22 & 22 \\
QED & 7 & 14 & 1 & 17 \\
Tau Polarisation & 33 & 14 & 16 & 23 \\
Tau branchings & 6 & 11 & 0 & 0 \\
Luminosity & 55 & 55 & - & - \\
\hline \hline Total uncertainty & 216 & 220 & 105 & 113 \\
\hline Correlated & 208 & 213 & 97 & 106 \\
Uncorrelated & 59 & 53 & 41 & 39 \\
\hline \hline TPC sector instability & 127 & 90 & 41 & 16 \\
\hline
\end{tabular}

Table 17: Systematic uncertainties for the 2000 data. All numbers in units of $10^{-4}$. The total uncertainties do not include the error due to TPC sector instability which applies only to a part of the 2000 data. The correlated error component includes errors correlated between energies and channels and those correlated with other LEP experiments. 
track selection every selection cut was varied in a wide range and the full analysis chain was repeated. If the observed change of result exceeded the expected statistical fluctuation $(1 \sigma)$ then the quadratic difference between the change and the expected fluctuation was taken as the systematic error, otherwise no systematic error was assigned. The largest uncertainty came from the impact parameters, $Z_{1,2}^{I M P}$, and from the combination of detectors which was used in the track fit.

The influence of event-selection cuts was checked in a similar way. Every eventselection cut was varied typically by the experimental resolution on the corresponding variable. The largest contribution came from the cuts on $\mathrm{E}_{\mathrm{VIS}}, \mathrm{P}_{\mathrm{RAD}}$ and electromagnetic energy. To reduce the effect of statistical fluctuations the full statistics of 1997-2000 were used to estimate the uncertainty from track and event selections and therefore these systematic errors were common and correlated for the data of different years.

The uncertainty of the detector calibration was mainly due to the limited statistics of test samples selected from real data of $\mathrm{Z}$ and high energy runs to estimate various corrections to the detector simulation. This part of the uncertainty was uncorrelated between different years. The second part of the uncertainty was due to residual disagreements in high energy data between real and simulated distributions used for calibration (correlated between different years). The largest contribution to the detector calibration uncertainty was the calibration of the HPC response.

The uncertainty of the residual background level consisted of three parts: the precision of the event generators (correlated); the statistics of the simulated background events (uncorrelated); and the uncertainty of the background level normalisation procedure. The normalisation uncertainty was determined from the statistics of real data events used for the normalisation (uncorrelated) and from residual data-simulation disagreements in the distributions of variables sensitive to particular background types (correlated). The uncertainties of the background from different sources are presented in Table 13.

Other (smaller) sources of systematic uncertainties were: statistics of simulated signal events; QED uncertainties computed by changing the order of perturbation theory to which QED corrections are included in the KK generator; subtraction of $\tau$ pairs accompanied by light pair production; reconstruction of $\sqrt{s^{\prime}}$; estimation of the feed-through event fraction; beam energy spread; trigger efficiency; world averages of $\tau$-decay branching ratios; and $\tau$-polarisation at LEP II energies.

An uncertainty specific to the measurements of the forward-backward asymmetries and differential cross-sections originated from the estimation of the charge misidentification probability. The systematic errors on the total non-radiative cross-section were assigned also to the differential cross-section. A correlation between the $\cos \theta$ bins was assumed for all sources except the statistics of simulated signal and simulated background. The charge misidentification uncertainty was assumed to be anti-correlated between the bins with $\cos \theta<0$ and $\cos \theta>0$.

A relatively small additional systematic uncertainty for the period of the broken TPC sector came from $\sqrt{s^{\prime}}$ reconstruction, trigger efficiency and charge identification. It was taken into account in the procedure of averaging of the two periods of 2000 .

\section{Cross-check with $\mathrm{Z}^{0}$ data}

For an additional cross-check of the data quality we have used the data taken in 19972000 during the LEP runs near the peak of the $\mathrm{Z}$ resonance. After the usual run selection the total integrated luminosity was about $11 \mathrm{pb}^{-1}$. Tau pairs were selected from this sample using a set of cuts very similar to the ones used for the high-energy analysis, and 
the total cross-section and forward-backward asymmetry were calculated using the same technique as for the high energies.

For the combined 1997-2000 sample the statistical uncertainties were $\Delta \sigma / \sigma= \pm 1.2 \%$ for the cross-section and $\Delta A_{F B}= \pm 0.012$ for the asymmetry estimation. Both values are similar to, or below, the systematic errors of the measurements at high energy.

The measured value of the forward-backward asymmetry was in good agreement with the Standard Model expectation, while the cross-section was found to be 1.1 standard deviations below the expected value (only the statistical error was taken into account). The year-by-year studies also did not show any significant departure from the expectations. These cross-checks give additional confidence that the measurements performed at the high energies are valid within the quoted uncertainties.

\section{4 q $\bar{q}$ final states}

The analysis of the $\mathrm{e}^{+} \mathrm{e}^{-} \rightarrow \mathrm{q} \overline{\mathrm{q}}(\gamma)$ process followed closely the procedure applied previously to the data collected near 183 and $189 \mathrm{GeV}$ and published in [4]. It benefited however from several improvements. In particular, more accurate generators were used for the simulation of the signal and background final states, and the enlarged Monte Carlo data sample allowed an improved understanding and control of residual differences between real and simulated distributions.

These improvements were implemented in the analysis of the latest data sets (collected above $190 \mathrm{GeV}$ ) and stimulated a reanalysis of the published data collected near 183 and $189 \mathrm{GeV}$. The magnitude of the changes as a result of these improvements did not justify reanalysing the data collected at 130-172 GeV [3], which have larger statistical uncertainties.

Two cross-sections were computed at each collision energy: an inclusive cross-section corresponding to reduced centre-of-mass energies larger than $10 \%$ of the collision energy, and a non-radiative cross-section corresponding to reduced centre-of-mass energies exceeding $85 \%$ of the collision energy.

\subsubsection{Analysis}

\section{Run selection}

The cross-section computations are based on data samples collected during running periods where the subdetectors of prime importance for the event selection were close enough to their nominal operating conditions. A small fraction of the data collected was discarded for this reason, mainly due to inefficiencies of the TPC, of the forward chambers (FCA-FCB) or of the FEMC. They correspond to about $1.4 \%$ of the total integrated luminosity.

\section{Track selection}

The selection of $q \bar{q}$ events relied mainly on charged particles for which the tracks were required to originate from the vertex (i.e. their transverse and longitudinal impact parameters with respect to the nominal interaction point had to be below $4 \mathrm{~cm}$ and $10 \mathrm{~cm}$ respectively), to have a momentum greater than $400 \mathrm{MeV} / c$ measured with a resolution better than 100\%, and to have a reconstructed track length of at least $30 \mathrm{~cm}$. For a small fraction of particle trajectories which were only reconstructed in the VD and ID, the length was required to exceed only $10 \mathrm{~cm}$. 


\section{DELPHI}

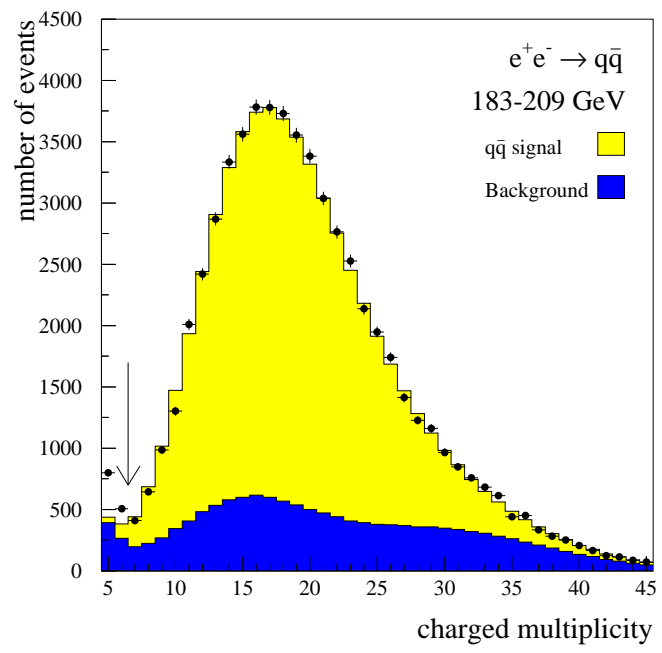

\section{DELPHI}

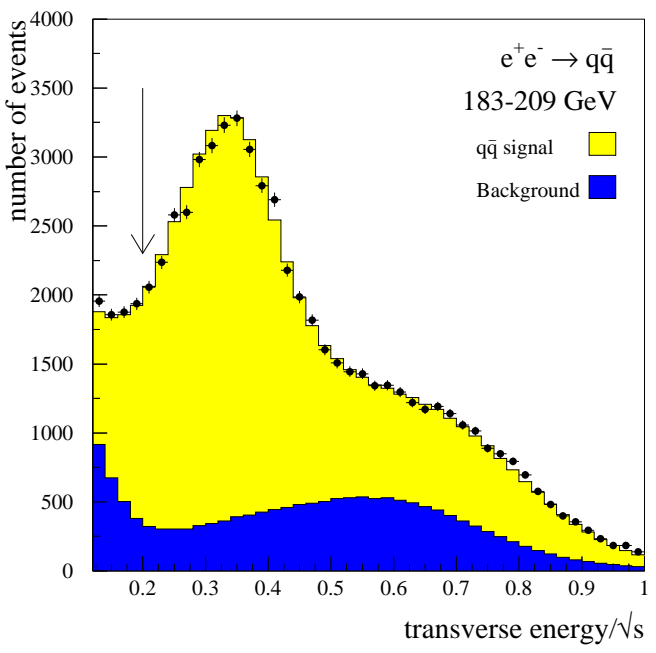

Figure 7: Charged multiplicity (left) and transverse energy (right) distributions for $\mathrm{e}^{+} \mathrm{e}^{-} \rightarrow \mathrm{q} \overline{\mathrm{q}}$ events. The arrows indicate the cut values applied. The distributions combine data collected from 1997 to 2000. 
DELPHI

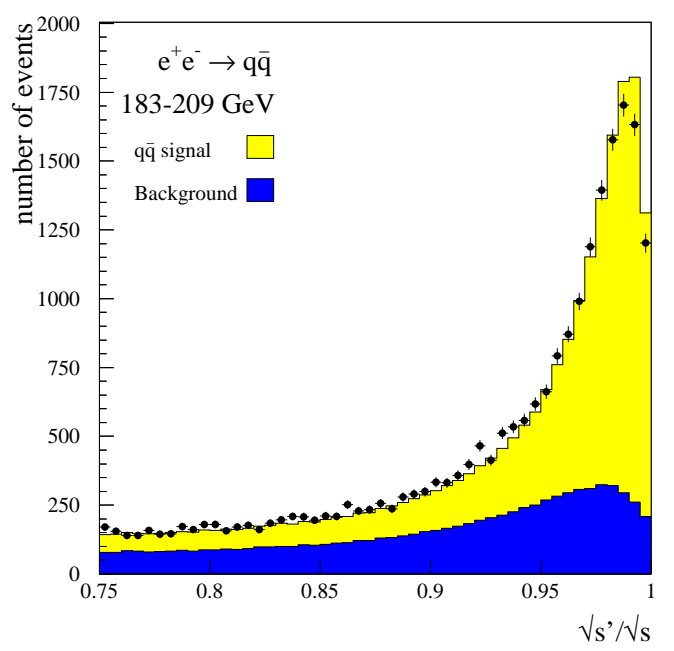

DELPHI

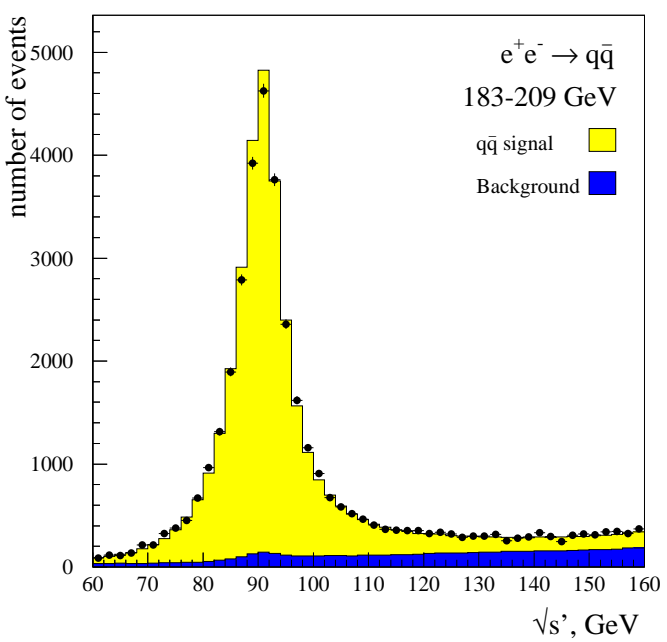

Figure 8: Reconstructed $\sqrt{s^{\prime}} / \sqrt{s}$ (left) and $\sqrt{s^{\prime}}$ (right) distributions for $\mathrm{e}^{+} \mathrm{e}^{-} \rightarrow \mathrm{q} \overline{\mathrm{q}}$ events. Data are from 1997-2000.

\section{Event selection}

The event selection was mainly oriented towards an efficient rejection of the backgrounds due to low multiplicity events and two-photon collisions. For this purpose, final states were accepted if they contained at least 7 selected tracks and if the total energy carried by the selected tracks exceeded $10 \%$ of the collision energy. In order to suppress the contamination by two-photon collisions further, the event total transverse energy was computed, based on the transverse momentum of each selected charged particle and on the electromagnetic showers reconstructed in the HPC and the FEMC with a shower energy above $500 \mathrm{MeV}$. Events were rejected if their total transverse energy was below $20 \%$ of the collision energy. Figure 7 shows the distributions of charged multiplicity and transverse energy for candidate $\mathrm{e}^{+} \mathrm{e}^{-} \rightarrow \mathrm{q} \overline{\mathrm{q}}$ events combined over the years 1997 to 2000 . Finally, a large fraction of the residual Bhabha events was discarded by requiring the

quadratic sum of the total energy (i.e. $E_{\text {rad }}=\sqrt{E_{\mathrm{F}}^{2}+E_{\mathrm{B}}^{2}}$ ) seen in each end-cap of the FEMC (computed with showers of at least $1 \mathrm{GeV}$ ) to be less than $90 \%$ of the beam energy. The sample of events satisfying the whole set of selection criteria described previously was thus mainly contaminated by four-fermion final states.

The selection of non-radiative final states included the additional requirement that the reduced centre-of-mass energy was larger than $85 \%$ of the collision energy (i.e. $\sqrt{s^{\prime}}>$ $0.85 \sqrt{s})$.

\section{$\sqrt{s^{\prime}}$ determination}

The determination of the reduced energy of each event $\left(\sqrt{s^{\prime}}\right)$ was mainly based on hadronic jets. A first step consisted in reconstructing jets according to the DURHAM clustering algorithm [30], using the selected charged particles as well as the neutral particles with energy larger than $1.5 \mathrm{GeV}$. A constrained fit was then performed, imposing energy and momentum conservation and assuming that an ISR photon was emitted along the beam lines. The free parameters of the fit were the jet directions and the ISR photon 


\begin{tabular}{|c|c|c|c|c|c|c|c|c|}
\hline \multicolumn{9}{|c|}{$\mathrm{e}^{+} \mathrm{e}^{-} \rightarrow \mathrm{q} \overline{\mathrm{q}}$} \\
\hline & \multicolumn{8}{|c|}{ Energy point $(\mathrm{GeV})$} \\
\hline & 183 & 189 & 192 & 196 & 200 & 202 & 205 & 207 \\
\hline Energy (GeV) & 182.7 & 188.6 & 191.6 & 195.5 & 199.5 & 201.6 & 204.9 & 206.5 \\
\hline Lumi $\left(\mathrm{pb}^{-1}\right)$ & 53.5 & 155.0 & 25.1 & 76.1 & 83.0 & 40.3 & 81.9 & 136.9 \\
\hline No. Events & 5859 & 15582 & 2433 & 7241 & 7452 & 3573 & 6792 & 10982 \\
\hline Efficiency (\%) & 92.3 & 92.3 & 92.4 & 92.3 & 92.5 & 92.4 & 92.1 & 92.2 \\
\hline Background (\%) & 26.7 & 30.3 & 31.2 & 32.3 & 34.4 & 35.2 & 36.6 & 37.6 \\
\hline
\end{tabular}

Table 18: The table shows the actual centre-of-mass energy and luminosity analysed at each energy point, the number of events selected in the inclusive samples and the efficiencies and backgrounds for the non-radiative selection.

energy $\left(E_{\gamma}\right)$. The value of $\sqrt{s^{\prime}}$ was then derived from the fitted value of $E_{\gamma}$ according to the following expression: $s^{\prime}=s-2 E_{\gamma} \sqrt{s}$.

The quality of the agreement between the real and simulated distributions of $\sqrt{s^{\prime}}$, which is essential for an accurate determination of the non-radiative event selection efficiency, was reasonable, as illustrated in Figure 8. The differences observed for $\sqrt{s^{\prime}}$ values close to $\sqrt{s}$ and to the $\mathrm{Z}$ mass reflect residual discrepancies between the real and simulated $\sqrt{s^{\prime}}$ resolutions. They have minor consequences on the analysis, which is mainly sensitive to differences occurring near the cut value of $0.85 \sqrt{s}$.

\section{Estimation of selection efficiency and backgrounds}

The selection efficiency was derived at each collision energy from the simulated sample of signal events satisfying the criteria above. The sample was generated with the KK program [17], the event hadronisation being performed with the PYTHIA algorithm [19, 20]. The selection efficiency values obtained for the inclusive sample varied from about $84 \%$ to $78 \%$ for increasing values of the collision energy. It remained near $92 \%$ at all collision energies for the non-radiative sample. Values of the selection efficiency at various centre-of-mass energies may be found in Table 18. The fraction of events generated below $85 \%$ of $\sqrt{s}$ and reconstructed above the cut value represented less than $10 \%$ of the nonradiative sample.

The residual background events contaminating the selected non-radiative (inclusive) sample originated mainly from WW pairs, which amounted to more than $90 \%$ (70\%) of the total background. The inclusive sample contained also significant contributions from $\mathrm{e}^{+} \mathrm{e}^{-} \rightarrow \mathrm{ZZ}, \mathrm{e}^{+} \mathrm{e}^{-} \rightarrow \mathrm{e}^{+} \mathrm{e}^{-} \mathrm{f} \overline{\mathrm{f}}$ and two-photon collision events. Other backgrounds, like those containing lepton pairs of opposite charge, amounted only to a few percent of the total residual background.

In order to assess the magnitude of the residual contamination of the non-radiative sample by WW events, which accounted for up to $1 / 3$ of the sample size, a dedicated study of the accuracy of the WW generator program (WPHACT) was performed. Variables reflecting the event shapes were multiplied to create a multi-variable selection parameter (see Figure 9), which was applied to the data in order to reject a substantial fraction of the background. The variables included: the energies of the most and least energetic jets; the minimal interjet angle; the broadening of the narrowest jet; the value of the clusterization parameter of the LUCLUS [20] algorithm at which the event changed from 3 to 4 jets. 


\section{DELPHI}

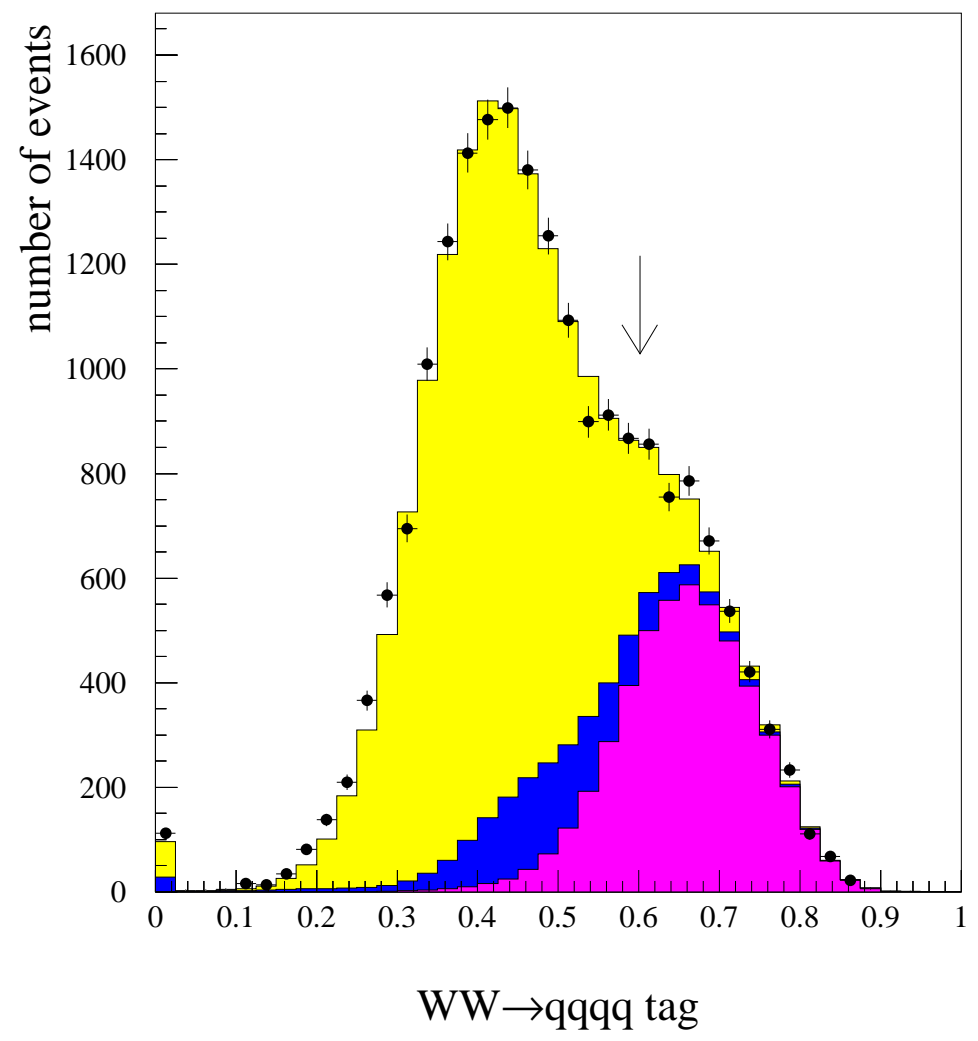

Figure 9: Distribution of the multi-variable selection parameter used to tag $W W$ events in the non-radiative sample. The light area represents the signal simulation, the darker area stands for the $W W \rightarrow q q q q$ background, and the darkest area displays the remaining backgrounds. The distributions combine data collected from 1997 to 2000.

About $60-70 \%$ of the residual WW events were discarded in this way, while the signal selection efficiency dropped by a few percent. The cross-sections computed before and after applying these additional rejection criteria differed by only small amounts (0.2-0.3 $\mathrm{pb}$ ), fully compatible with the statistical uncertainty affecting the comparisons.

\section{Forward region simulation}

The simulated rate of tracks belonging to multi-hadronic final states and reconstructed in the forward regions of the detector (i.e. with a polar angle between $9^{\circ}$ and $30^{\circ}$ or between $150^{\circ}$ and $171^{\circ}$ ) underestimated the observed rate by up to $10 \%$. This deficit was regarded as a consequence of the approximations made in the description of the forward material of the detector at the simulation level.

In order to account for the deficit, an artificial inefficiency was applied to the real tracks reconstructed in the forward regions. It consisted in discarding randomly some of these tracks, with weights extracted from the ratio between the real and simulated track polar angle distributions in four different momentum bins. These corrections were extracted from the calibration data collected each year near the $\mathrm{Z}$ resonance peak and applied to the high-energy data of the same year. The corresponding change of the inclusive and nonradiative cross-sections was $-1.2 \%$ on average. The associated systematic uncertainty was estimated as one third of the change (i.e. $0.4 \%$ ). 
An alternative treatment was used as a cross-check. It consisted in assigning weights to the simulated forward tracks in order to reproduce the excess of the real ones. This approach resulted in values of the inclusive cross-section well consistent with those obtained with the previous method, which was however preferred for the cross-section determination as it was significantly simpler to implement in the $\sqrt{s^{\prime}}$ reconstruction algorithm.

\section{Impact of the TPC instability in the year 2000}

The failure of one of the TPC sectors in the year 2000 translated into a small loss in the reconstruction efficiency for tracks traversing the sector affected, which was estimated to occur in about $40 \%$ of multihadronic events. According to the event simulation, the corresponding drop of the event selection efficiency for the inclusive sample was moderate $(-0.6 \%)$. Several cross-checks were performed in order to test how accurately the simulation reproduced the modified track and jet reconstructions. While the rate of real tracks was reproduced quite well, residual differences were found between real and simulated jet parameters, which were accounted for by a specific systematic uncertainty. The latter was estimated as $\pm 0.1 \%$ for the inclusive sample selection efficiency and $\pm 0.5 \%$ for the non-radiative sample selection efficiency.

\section{Subtraction of interference between ISR and FSR}

Computations performed with the ZFITTER package showed that the inclusion of the ISR-FSR interference in the computation of the non-radiative cross-section diminishes its magnitude by almost $0.5 \%$.

Since the KK generator used for the simulation of multi-hadronic final states did not account for interference between initial and final state photon radiation, and since the expected change in the cross-section is not uniform, peaking at $|\cos \theta|=1$, where the efficiency is smallest, the quark polar angle distribution was reweighted by the relative difference in the differential cross-sections computed from ZFITTER, with and without ISR-FSR interference. The change in the selection efficiency was computed, and found to be negligible. Thus the measured non-radiative cross-sections were corrected by the full $0.5 \%$ correction.

\subsubsection{Results}

\section{Determination of the cross-sections}

The $\mathrm{e}^{+} \mathrm{e}^{-} \rightarrow \mathrm{q} \overline{\mathrm{q}}$ cross-sections were derived from the expression:

$$
\sigma_{q q}=\frac{\left(N_{s e l}-N_{b g}\right)(1-f)}{\epsilon \mathcal{L}}
$$

where $N_{s e l}$ stands for the number of events selected, $N_{b g}$ is the number of background events expected, $\epsilon$ is the selection efficiency and $\mathcal{L}$ stands for the integrated luminosity. The term $(1-f)$ applies only to the non-radiative cross-section, the parameter $f$ expressing the feed-up by events produced at a reduced centre-of-mass energy below $85 \%$ of $\sqrt{s}$.

The cross-section values found at each energy point are presented in Table 19 and shown in Figure 10, together with the Standard Model predictions computed with the ZFITTER program. The non-radiative cross-sections at energies from $130 \mathrm{GeV}$ to $172 \mathrm{GeV}$ supersede those of [3] because of the correction for ISR-FSR interference mentioned in Section 5.4.1. 


\begin{tabular}{|c|c|c|c|c|c|}
\hline \multicolumn{6}{|c|}{$\mathrm{e}^{+} \mathrm{e}^{-} \rightarrow \mathrm{q} \overline{\mathrm{q}}$} \\
\hline \multirow{2}{*}{$\begin{array}{c}\sqrt{s} \\
(\mathrm{GeV})\end{array}$} & $\sqrt{s^{\prime}} / \sqrt{s}>0.10$ & $\sqrt{s^{\prime}} / \sqrt{s}>0.85$ & \multirow{2}{*}{$\begin{array}{c}\sqrt{s} \\
(\mathrm{GeV}) \\
\end{array}$} & $\sqrt{s^{\prime}} / \sqrt{s}>0.10$ & $\sqrt{s^{\prime}} / \sqrt{s}>0.85$ \\
\hline & $\sigma_{q q}(\mathrm{pb})$ & $\sigma_{q q}(\mathrm{pb})$ & & $\sigma_{q q}(\mathrm{pb})$ & $\sigma_{q q}(\mathrm{pb})$ \\
\hline 130 & $\begin{array}{c}328.4 \pm 11.3 \pm 3.7 \\
(328.1)\end{array}$ & $\begin{array}{c}82.4 \pm 5.2 \pm 2.6 \\
(82.5)\end{array}$ & 192 & $\begin{array}{c}92.9 \pm 2.4 \pm 1.0 \\
(93.4)\end{array}$ & $\begin{array}{c}22.1 \pm 1.1 \pm 0.3 \\
(21.2)\end{array}$ \\
\hline 136 & $\begin{array}{c}259.6 \pm 10.0 \pm 3.1 \\
(272.0)\end{array}$ & $\begin{array}{c}65.3 \pm 4.7 \pm 2.1 \\
(66.4)\end{array}$ & 196 & $\begin{array}{c}91.1 \pm 1.4 \pm 0.9 \\
(88.7)\end{array}$ & $\begin{array}{c}21.2 \pm 0.6 \pm 0.3 \\
(20.1)\end{array}$ \\
\hline 161 & $\begin{array}{c}158.3 \pm 4.4 \pm 2.0 \\
(151.4)\end{array}$ & $\begin{array}{c}41.0 \pm 2.1 \pm 1.3 \\
(35.1)\end{array}$ & 200 & $\begin{array}{c}85.2 \pm 1.3 \pm 0.9 \\
(84.2) \\
\end{array}$ & $\begin{array}{c}19.5 \pm 0.6 \pm 0.3 \\
(19.0) \\
\end{array}$ \\
\hline 172 & $\begin{array}{c}125.5 \pm 4.2 \pm 1.9 \\
(125.1)\end{array}$ & $\begin{array}{c}30.4 \pm 1.9 \pm 1.0 \\
(28.7)\end{array}$ & 202 & $\begin{array}{c}84.2 \pm 1.9 \pm 0.9 \\
(82.0)\end{array}$ & $\begin{array}{c}18.9 \pm 0.8 \pm 0.3 \\
(18.5)\end{array}$ \\
\hline 183 & $\begin{array}{c}107.6 \pm 1.7 \pm 1.0 \\
(106.0)\end{array}$ & $\begin{array}{c}25.5 \pm 0.8 \pm 0.3 \\
(24.2)\end{array}$ & 205 & $\begin{array}{c}77.8 \pm 1.3 \pm 0.9 \\
(78.9)\end{array}$ & $\begin{array}{c}17.7 \pm 0.6 \pm 0.3 \\
(17.8)\end{array}$ \\
\hline 189 & $\begin{array}{c}96.9 \pm 1.0 \pm 0.9 \\
(97.3)\end{array}$ & $\begin{array}{c}22.6 \pm 0.5 \pm 0.3 \\
(22.1)\end{array}$ & 207 & $\begin{array}{c}74.7 \pm 1.0 \pm 0.8 \\
(77.3)\end{array}$ & $\begin{array}{c}17.0 \pm 0.4 \pm 0.3 \\
(17.4)\end{array}$ \\
\hline
\end{tabular}

Table 19: Measured values of the inclusive and non-radiative cross-sections for the process $\mathrm{e}^{+} \mathrm{e}^{-} \rightarrow \mathrm{q} \overline{\mathrm{q}}$ at collision energies ranging from 130 to $207 \mathrm{GeV}$. The uncertainties include the statistical and all systematic contributions. Values in parentheses are the Standard Model predictions computed with the ZFITTER program, and are estimated to have a precision of $\pm 0.26 \%$. 


\section{Systematic uncertainty}

Most of the systematic uncertainties on the selection efficiency resulted from small residual discrepancies between real and simulated distributions. Because of these differences, the selection cuts were expected to have slightly different effects on the simulated distributions than on the real ones. The associated uncertainties were estimated from the observed changes of the cross-section when varying the cut values. They added up to a total contribution of $1.0 \%$.

The systematic uncertainty related to the ISR modelling was estimated by comparing the selection efficiencies extracted from two different simulation samples, produced either with the KK or with the PYTHIA generators. The observed difference in the selection efficiency was very small, i.e. $0.1 \%$, and was converted into a systematic uncertainty of identical size.

The uncertainty related to the accuracy of the fragmentation modelling in the Monte Carlo was derived from the comparison between a sample where the hadronisation was performed with PYTHIA and a sample where it was done with ARIADNE (version 4.08) [31], with parameters tuned to DELPHI data [21]. The difference between the two values of the selection efficiency (0.1-0.25\%) was taken as the corresponding systematic uncertainty.

A $0.7 \%$ systematic uncertainty was assigned to the non-radiative cross-section as a consequence of the cut on $\sqrt{s^{\prime}}$. Its value follows from the resolution attributed to the reconstructed value of $\sqrt{s^{\prime}}$.

The systematic uncertainty on the residual backgrounds accounts for the theoretical precision of the generators used and for the accuracy on the selection efficiency associated to each final state. The uncertainty affecting the non-radiative cross-section was completely dominated by the precision on the WW background estimation, while the inclusive cross-section was also affected by the uncertainties related to the subtraction of residual contaminations due to $\mathrm{e}^{+} \mathrm{e}^{-} \rightarrow \mathrm{ZZ}, \mathrm{e}^{+} \mathrm{e}^{-} \rightarrow \mathrm{e}^{+} \mathrm{e}^{-} \mathrm{f} \overline{\mathrm{f}}$ and two-photon collision final states.

The quadratic combination of the contributions to the systematic uncertainties described above translate into a total uncertainty on the selection efficiency amounting to $1.1 \%$ for the inclusive cross-section and $1.3 \%$ for the non-radiative cross-sections. As for the residual backgrounds, a $0.5 \mathrm{pb}(0.2 \mathrm{pb})$ total uncertainty was assigned to the inclusive (non-radiative) cross-section. The correlation between systematic uncertainties assigned to cross-sections measured at different energy points was estimated as $80 \%$ when coming from the event selection, and as $100 \%$ when originating from the residual background subtraction.

The breakdown of the systematic uncertainties on the $\mathrm{e}^{+} \mathrm{e}^{-} \rightarrow \mathrm{q} \overline{\mathrm{q}}$ cross-sections is provided in Table 20.

\subsection{Summary of results}

The measurements of cross-sections and forward-backward asymmetries for nonradiative samples of events are compared to theoretical predictions in Figures 10 and 11. In both cases the measurements are compared directly to the predictions and the ratios of the measurements to the prediction are shown,

$$
\mathcal{R}=\frac{\mathcal{O}}{\mathcal{O}_{S M}}
$$




\begin{tabular}{|l|c||c|}
\hline \multicolumn{3}{|c|}{$\mathrm{e}^{+} \mathrm{e}^{-} \rightarrow \mathrm{q} \overline{\mathrm{q}}$} \\
\hline \hline Source & $\begin{array}{c}\Delta \sigma / \sigma \\
\text { (non-rad.) }\end{array}$ & $\begin{array}{c}\Delta \sigma / \sigma \\
\text { (inclus.) }\end{array}$ \\
\hline \hline Forward corrections & 40 & 40 \\
Selection cuts & 100 & 100 \\
ISR modelling & 10 & 10 \\
Fragmentation & 25 & 10 \\
$\sqrt{s^{\prime}}$ cut & 70 & - \\
Backgrounds & 120 & 70 \\
Luminosity & 55 & 55 \\
TPC sector instability & 50 & 10 \\
\hline \hline Total uncertainty & 186 & 140 \\
\hline Correlated & 169 & 124 \\
Uncorrelated & 79 & 65 \\
\hline
\end{tabular}

Table 20: Breakdown of the systematic uncertainties on the $\mathrm{e}^{+} \mathrm{e}^{-} \rightarrow \mathrm{q} \overline{\mathrm{q}}$ cross-sections for data taken at $\sqrt{s} \sim 207 \mathrm{GeV}$. All numbers in units of $10^{-4}$. The total uncertainties do not include the uncertainty due to the TPC instability, which applies only to a part of the data collected in 2000. The correlated error component includes errors correlated between energies and channels and those correlated with other LEP experiments. 
DELPHI

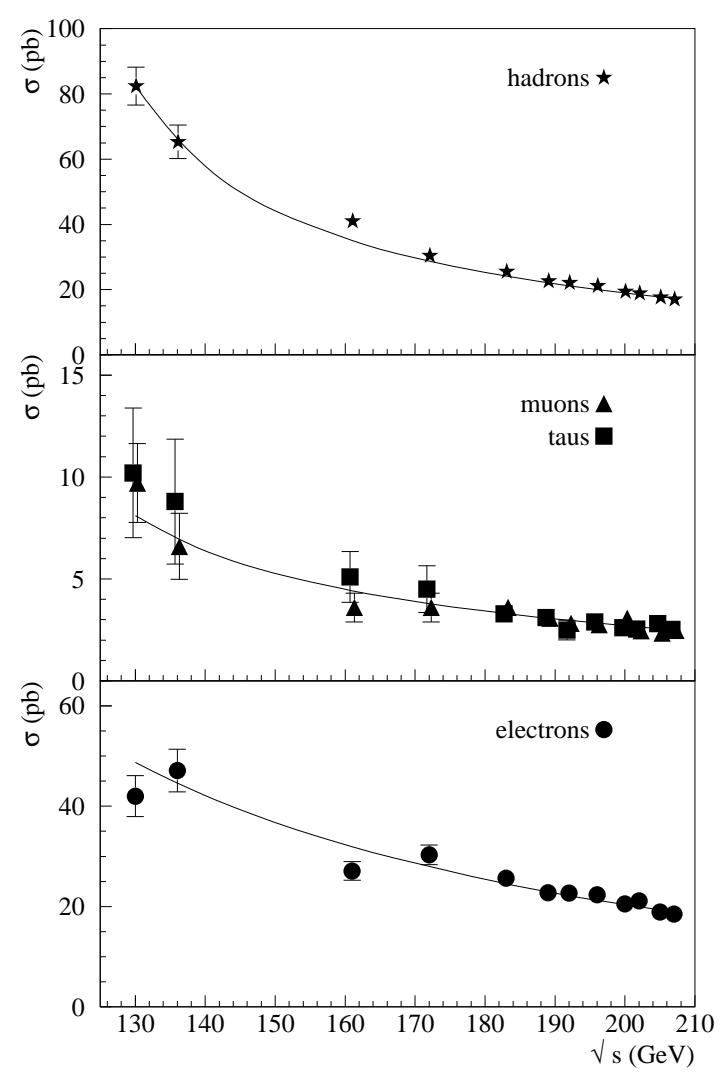

DELPHI

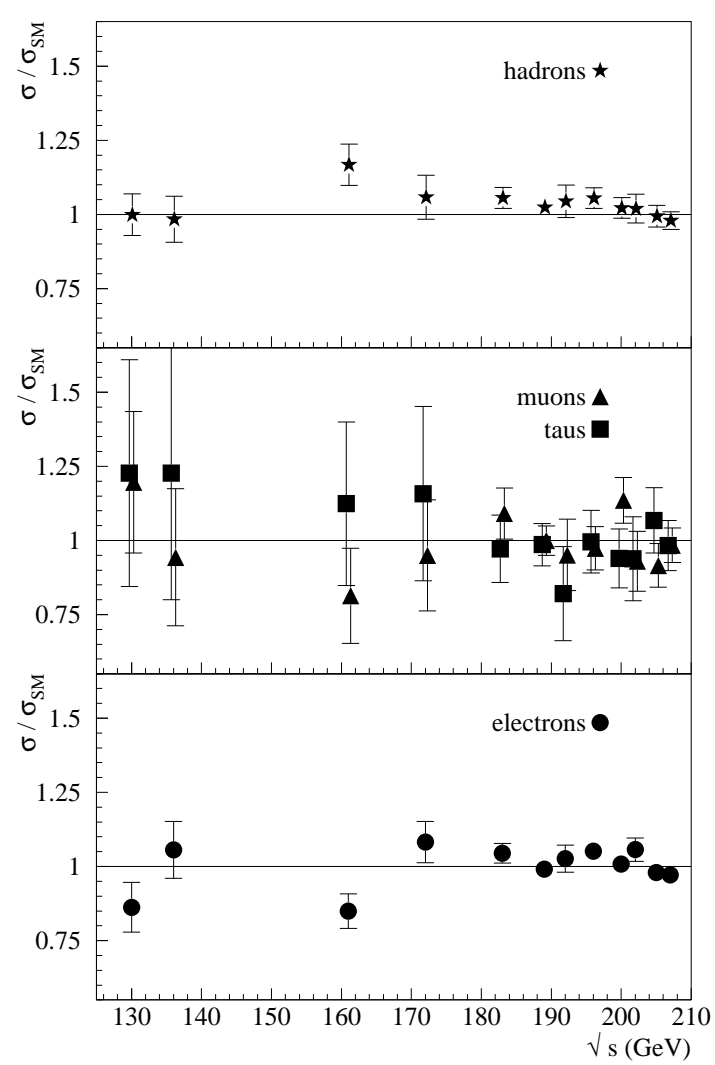

Figure 10: Comparisons of the measurements of cross-sections to predictions of the Standard Model from BHWIDE and ZFITTER for non-radiative samples of $\mathrm{e}^{+} \mathrm{e}^{-}, \mu^{+} \mu^{-}$, $\tau^{+} \tau^{-}$and $\mathrm{q} \overline{\mathrm{q}}$ final states. The measurements are compared to the predictions shown as curves (left) and as values of $\mathcal{R}$ the ratio of the measurement to the predictions (right). For clarity the results for $\mu^{+} \mu^{-}$and $\tau^{+} \tau^{-}$final states are slightly displaced from the actual centre-of-mass energy of the data. The errors include the statistical and experimental systematic errors. 
DELPHI

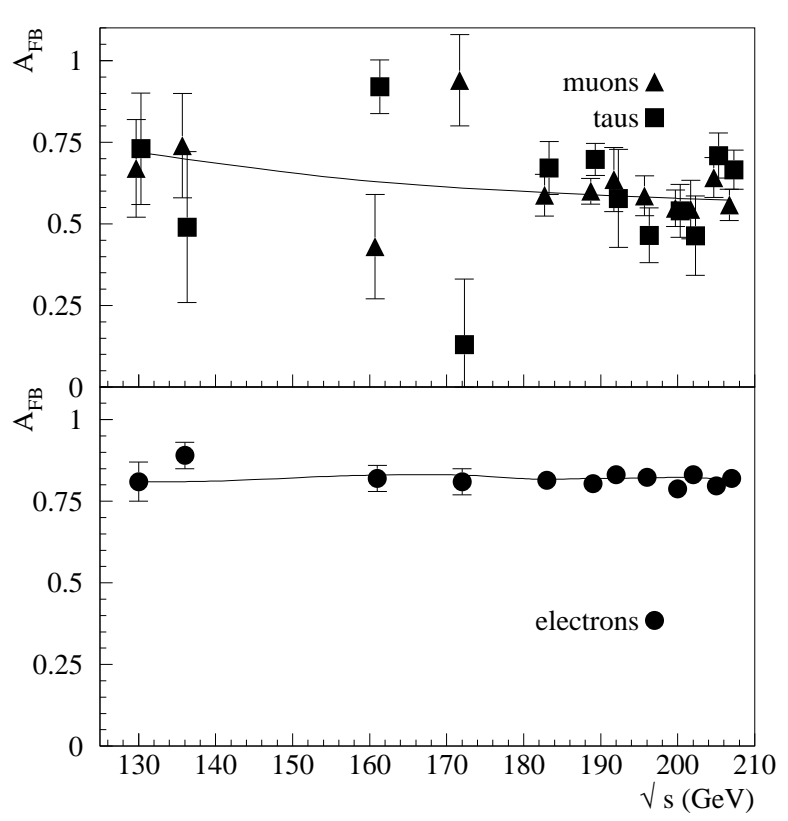

DELPHI

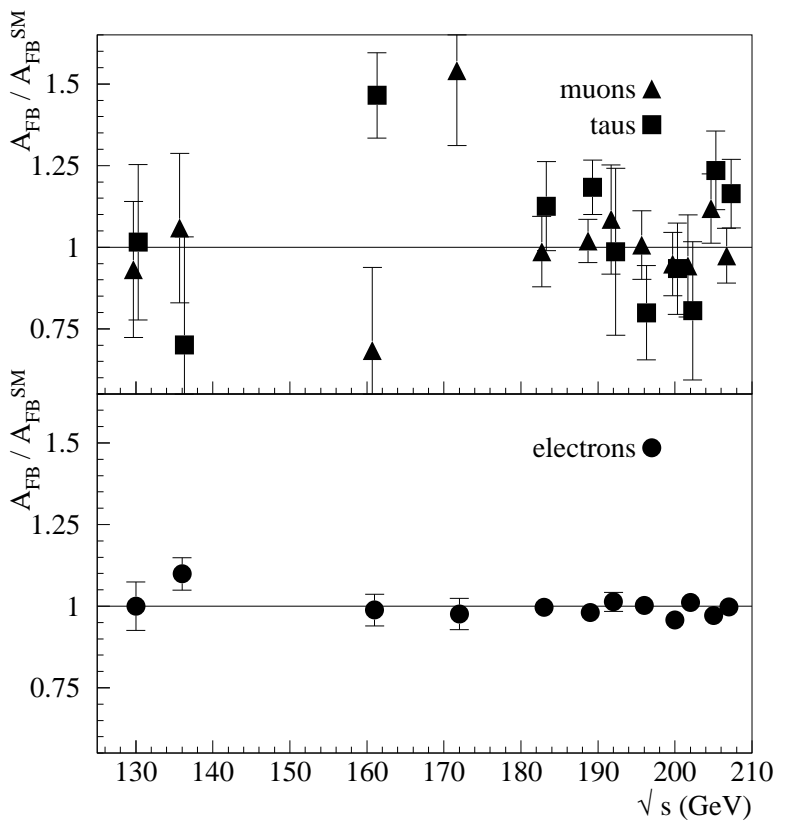

Figure 11: Comparisons of the measurements of forward-backward asymmetries to predictions of the Standard Model from BHWIDE and ZFITTER for non-radiative samples of $\mathrm{e}^{+} \mathrm{e}^{-}, \mu^{+} \mu^{-}$and $\tau^{+} \tau^{-}$final states. The measurements are compared to the predictions shown as curves (left) and as values of $\mathcal{R}$ the ratio of the measurement to the predictions (right). For clarity the results for $\mu^{+} \mu^{-}$and $\tau^{+} \tau^{-}$final states are slightly displaced from the actual centre-of-mass energy of the data. The errors include the statistical and experimental systematic errors. 


\begin{tabular}{|c|c|c|}
\hline \multirow{3}{*}{ Meas. } & $\begin{array}{c}\text { Final } \\
\text { States }\end{array}$ & $\langle\mathcal{R}\rangle \pm($ stat $) \pm($ syst $) \pm($ theory $)$ \\
\hline \hline$\sigma$ & $\mathrm{e}^{+} \mathrm{e}^{-}$ & $1.0006 \pm 0.0086 \pm 0.0077 \pm 0.0200$ \\
\cline { 2 - 3 } & $\mu^{+} \mu^{-}$ & $0.9961 \pm 0.0244 \pm 0.0062 \pm 0.0040$ \\
\cline { 2 - 3 } & $\tau^{+} \tau^{-}$ & $0.9852 \pm 0.0341 \pm 0.0203 \pm 0.0040$ \\
\cline { 2 - 3 } & $\mathrm{q} \overline{\mathrm{q}}$ & $1.0256 \pm 0.0103 \pm 0.0130 \pm 0.0026$ \\
\cline { 2 - 3 } & $\mathrm{l}^{+} \mathrm{l}^{-}$ & $0.9930 \pm 0.0200 \pm 0.0074 \pm 0.0040$ \\
\hline \multirow{3}{*}{$\mathrm{A}_{\mathrm{FB}}$} & $\mathrm{e}^{+} \mathrm{e}^{-}$ & $0.9896 \pm 0.0061 \pm 0.0043 \pm 0.0244$ \\
\cline { 2 - 3 } & $\mu^{+} \mu^{-}$ & $1.0120 \pm 0.0335 \pm 0.0010 \pm 0.0068$ \\
\cline { 2 - 3 } & $\tau^{+} \tau^{-}$ & $1.1121 \pm 0.0405 \pm 0.0156 \pm 0.0068$ \\
\cline { 2 - 3 } & $\mathrm{l}^{+} \mathrm{l}^{-}$ & $1.0494 \pm 0.0259 \pm 0.0059 \pm 0.0068$ \\
\hline
\end{tabular}

Table 21: Average values of $\mathcal{R}$ for cross-sections and forward-backward asymmetries for $\mathrm{e}^{+} \mathrm{e}^{-}, \mu^{+} \mu^{-}, \tau^{+} \tau^{-}$and $\mathrm{q} \overline{\mathrm{q}}$ final states. The errors on the averages, $\langle\mathcal{R}\rangle$, are the statistical, experimental systematic and theoretical uncertainties.

where $\mathcal{O}$ is the measured observable and $\mathcal{O}_{S M}$ is the prediction for the observable. A useful guide to the compatibility of the different sets of measurements with the theoretical predictions is to average the ratios of the measured results to the predictions over all centre-of-mass energies, $\langle\mathcal{R}\rangle$. Such averages have been calculated for the measurements made on non-radiative samples of events:

- cross-sections for $\mathrm{e}^{+} \mathrm{e}^{-}, \mu^{+} \mu^{-}, \tau^{+} \tau^{-}, \mathrm{q} \overline{\mathrm{q}}$ final states and $\mathrm{l}^{+} \mathrm{l}^{-}$, an average of the combination of the $\mu^{+} \mu^{-}$and $\tau^{+} \tau^{-}$final states; and

- forward-backward asymmetries for $\mathrm{e}^{+} \mathrm{e}^{-}, \mu^{+} \mu^{-}, \tau^{+} \tau^{-}$and $\mathrm{l}^{+} \mathrm{l}^{-}$final states; and

- bin-by-bin averages of the differential cross-sections for $\mu^{+} \mu^{-}$and $\tau^{+} \tau^{-}$final states.

The averages were made using the BLUE technique [29] so that correlations between systematic errors in the different averages were taken into account. The combination method makes it easy to identify the statistical and systematic uncertainties on $\langle\mathcal{R}\rangle$, for each set of measurements. For the cross-section and forward-backward asymmetry measurements the averages are made over all centre-of-mass energies from 130 to $207 \mathrm{GeV}$.

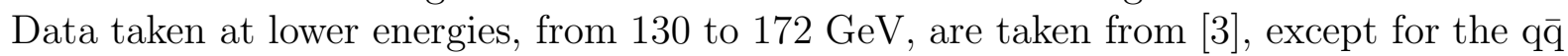
final state where the updated values of Table 19 are used. The experimental systematic errors for the lower energy data, with the exception of luminosity errors, are taken to be correlated amongst themselves for each measurement, and uncorrelated with the errors at higher energies. Theoretical uncertainties on the cross-sections and asymmetries are taken from [32]. For the differential cross-section the averages are made over energies from 183-207 GeV, and for the $\mu^{+} \mu^{-}$and $\tau^{+} \tau^{-}$final states the statistical error is taken from the expected error. The relative theoretical uncertainties on the differential crosssections are taken to be the same as the relative uncertainties on the cross-sections as given in [32], and assumed to be fully correlated between bins.

Results for the averages of $\langle\mathcal{R}\rangle$ for cross-sections and forward-backward asymmetries are given in Table 21. They indicate satisfactory agreement between the data and the predictions, with the largest deviation from the expectation being approximately 2.6 standard deviations. In most cases the uncertainties on the averages are dominated by statistical errors, except for the cross-section and forward-backward asymmetry for $\mathrm{e}^{+} \mathrm{e}^{-} \rightarrow \mathrm{e}^{+} \mathrm{e}^{-}$ where the theoretical errors dominate, and for the cross-section for $\mathrm{e}^{+} \mathrm{e}^{-} \rightarrow \mathrm{q} \overline{\mathrm{q}}$ where the experimental systematic error dominates. 


\begin{tabular}{|c|c|}
\hline \multicolumn{2}{|c|}{$\mathrm{e}^{+} \mathrm{e}^{-} \rightarrow \mathrm{e}^{+} \mathrm{e}^{-}$} \\
\hline \hline $\cos \theta$ & $\langle\mathcal{R}\rangle \pm$ (stat) \pm (syst) \pm (theory) \\
\hline$[-0.72,-0.54]$ & $1.106 \pm 0.091 \pm 0.049 \pm 0.020$ \\
{$[-0.54,-0.36]$} & $1.077 \pm 0.074 \pm 0.014 \pm 0.020$ \\
{$[-0.36,-0.18]$} & $0.951 \pm 0.060 \pm 0.008 \pm 0.020$ \\
{$[-0.18,0.00]$} & $1.113 \pm 0.049 \pm 0.026 \pm 0.020$ \\
{$[0.00,0.09]$} & $1.005 \pm 0.047 \pm 0.027 \pm 0.020$ \\
{$[0.09,0.18]$} & $0.999 \pm 0.055 \pm 0.007 \pm 0.020$ \\
{$[0.18,0.27]$} & $0.958 \pm 0.046 \pm 0.007 \pm 0.020$ \\
{$[0.27,0.36]$} & $0.943 \pm 0.038 \pm 0.007 \pm 0.020$ \\
{$[0.36,0.45]$} & $1.030 \pm 0.033 \pm 0.008 \pm 0.020$ \\
{$[0.45,0.54]$} & $0.974 \pm 0.026 \pm 0.008 \pm 0.020$ \\
{$[0.54,0.63]$} & $1.007 \pm 0.021 \pm 0.008 \pm 0.020$ \\
{$[0.63,0.72]$} & $0.999 \pm 0.016 \pm 0.008 \pm 0.020$ \\
\hline
\end{tabular}

\begin{tabular}{|c|c|}
\hline \multicolumn{2}{|c|}{$\mathrm{e}^{+} \mathrm{e}^{-} \rightarrow \mu^{+} \mu^{-}$} \\
\hline \hline $\cos \theta$ & $\langle\mathcal{R}\rangle \pm($ stat$) \pm$ (syst) \pm (theory) \\
\hline$[-0.97,-0.80]$ & $1.166 \pm 0.150 \pm 0.009 \pm 0.004$ \\
{$[-0.80,-0.60]$} & $0.964 \pm 0.138 \pm 0.008 \pm 0.004$ \\
{$[-0.60,-0.40]$} & $1.092 \pm 0.127 \pm 0.008 \pm 0.004$ \\
{$[-0.40,-0.20]$} & $0.761 \pm 0.112 \pm 0.007 \pm 0.004$ \\
{$[-0.20,0.00]$} & $1.031 \pm 0.098 \pm 0.007 \pm 0.004$ \\
{$[0.00,0.20]$} & $1.004 \pm 0.085 \pm 0.007 \pm 0.004$ \\
{$[0.20,0.40]$} & $1.067 \pm 0.072 \pm 0.007 \pm 0.004$ \\
{$[0.40,0.60]$} & $1.083 \pm 0.063 \pm 0.006 \pm 0.004$ \\
{$[0.60,0.80]$} & $0.933 \pm 0.056 \pm 0.006 \pm 0.004$ \\
{$[0.80,0.97]$} & $1.003 \pm 0.054 \pm 0.006 \pm 0.004$ \\
\hline
\end{tabular}

\begin{tabular}{|c|c|}
\hline \multicolumn{2}{|c|}{$\mathrm{e}^{+} \mathrm{e}^{-} \rightarrow \tau^{+} \tau^{-}$} \\
\hline \hline $\cos \theta$ & $\langle\mathcal{R}\rangle \pm$ (stat) \pm (syst) \pm (theory) \\
\hline$[-0.96,-0.80]$ & $0.397 \pm 0.291 \pm 0.025 \pm 0.004$ \\
{$[-0.80,-0.60]$} & $0.757 \pm 0.221 \pm 0.031 \pm 0.004$ \\
{$[-0.60,-0.40]$} & $0.970 \pm 0.166 \pm 0.023 \pm 0.004$ \\
{$[-0.40,-0.20]$} & $0.898 \pm 0.144 \pm 0.022 \pm 0.004$ \\
{$[-0.20,0.00]$} & $0.972 \pm 0.133 \pm 0.023 \pm 0.004$ \\
{$[0.00,0.20]$} & $1.122 \pm 0.111 \pm 0.021 \pm 0.004$ \\
{$[0.20,0.40]$} & $1.106 \pm 0.089 \pm 0.020 \pm 0.004$ \\
{$[0.40,0.60]$} & $0.967 \pm 0.078 \pm 0.021 \pm 0.004$ \\
{$[0.60,0.80]$} & $0.875 \pm 0.086 \pm 0.022 \pm 0.004$ \\
{$[0.80,0.96]$} & $1.027 \pm 0.098 \pm 0.021 \pm 0.004$ \\
\hline
\end{tabular}

Table 22: Bin-by-bin average values of $\mathcal{R}$ for differential cross-sections for $\mathrm{e}^{+} \mathrm{e}^{-}, \mu^{+} \mu^{-}$ and $\tau^{+} \tau^{-}$final states. The errors on the averages, $\langle\mathcal{R}\rangle$, are the statistical, experimental systematic and theoretical uncertainties. 


\section{DELPHI}
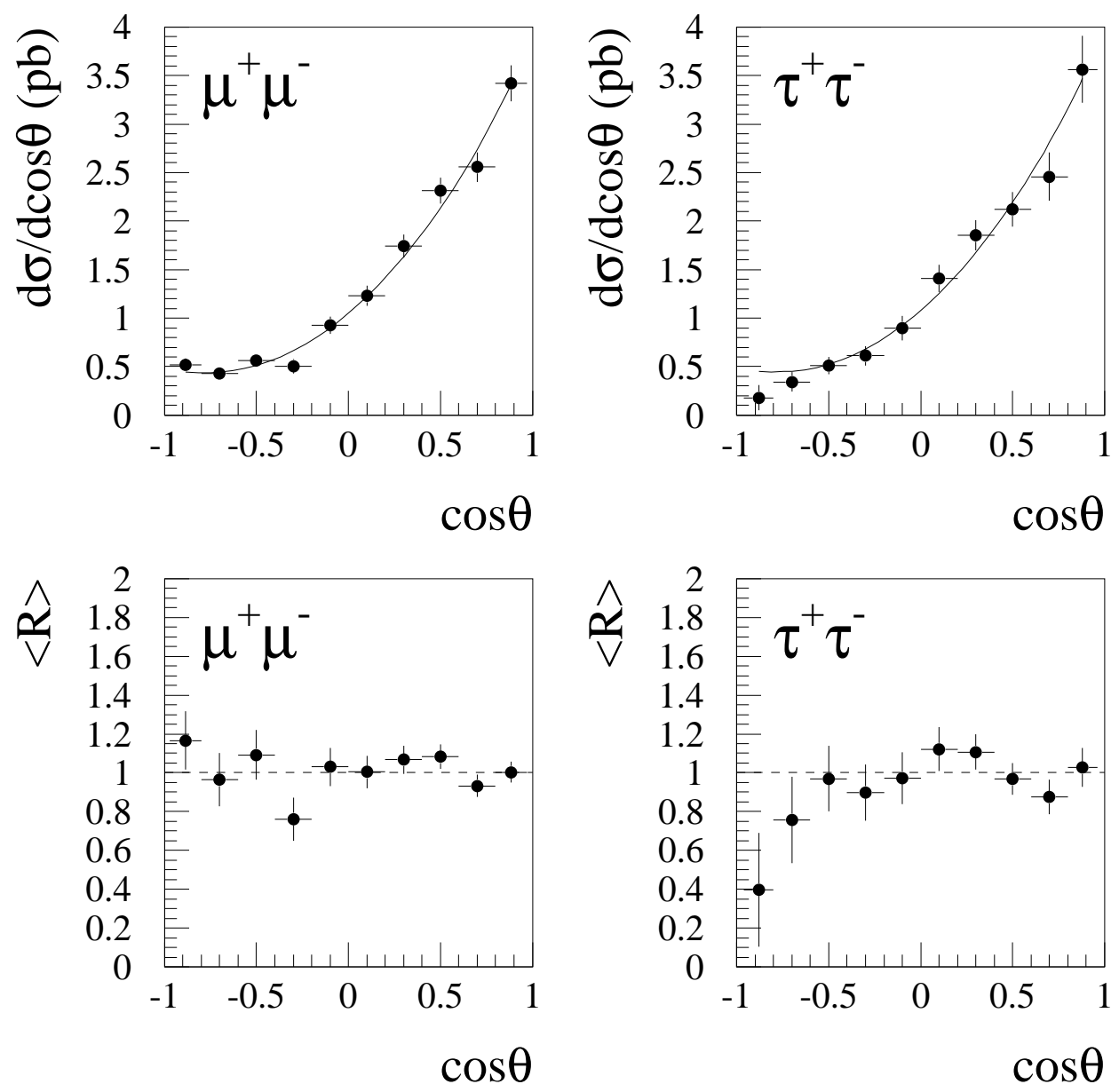

Figure 12: Bin-by-bin averages, $\langle\mathcal{R}\rangle$, for $\mu^{+} \mu^{-}$and $\tau^{+} \tau^{-}$final states. The lower plots show the values of $\langle\mathcal{R}\rangle$ while the upper plots show the $S M \mathrm{~d} \sigma / \mathrm{d} \cos \theta$ multiplied by $\langle\mathcal{R}\rangle$ at the luminosity weighted centre-of-mass energy for each measurement channel. 
Results for the bin-by-bin averages of ratios of the measured differential cross-sections to the predictions are given in Table 22. These are shown in Figure 12, in which the averaged ratios have been expressed as a differential cross-section at the luminosity weighted centre-of-mass energy for the $\mu^{+} \mu^{-}$and $\tau^{+} \tau^{-}$final states by multiplying the averaged ratios, $\langle\mathcal{R}\rangle$, by the predicted differential cross-sections in each bin at the appropriate centre-of-mass energy. The results show good agreement with the expectation.

Overall the data show good agreement between the measurements and the predictions of the Standard Model from ZFITTER and BHWIDE. In the following section the measurements are interpreted in a variety of models which allow for physics beyond the Standard Model. Since each of these models predicts specific behaviours for either the differential cross-section, or alternatively cross-sections and forward-backward asymmetries, as a function of centre-of-mass energy, the predictions of the models are compared directly to the individual measurements rather than to the averaged $\langle\mathcal{R}\rangle$ values given in this section.

\section{Interpretation}

The results of the measurements of cross-sections, forward-backward asymmetries and angular distributions presented in Section 5 have been interpreted in a variety of scenarios. The data were used to determine the parameters of the S-matrix formalism for $\mathrm{e}^{+} \mathrm{e}^{-} \rightarrow \mathrm{f} \bar{f}$, as well as to investigate a variety of models which include explicit forms of physics beyond the Standard Model ${ }^{4}$ : models with $\mathrm{Z}^{\prime}$ bosons, contact interactions, models which include the exchange of gravitons in large extra dimensions and models which consider possible $s$ or $t$ channel sneutrino $\tilde{\nu}_{l}$ exchange in R-parity violating supersymmetry. For the S-matrix ansatz and the search for $\mathrm{Z}^{\prime}$ bosons the LEP II data reported here were used together with measurements from LEP I. For other studies, the LEP II data alone were used.

In the following subsections, each of these interpretations is discussed. In all cases the theoretical basis of the model is summarised and the relevant parameters are identified. The methods of fitting parameters of the models to the data are discussed. The theoretical uncertainties on the Standard Model predictions for cross-sections and asymmetries, as given in Section 5, are explicitly included in the fits. In most cases, the relevant papers discuss the predictions of the models only at Born level, however, to confront the models with data it is necessary to take into account QED radiative corrections. The methods used to implement radiative corrections are described for each model. In the absence of evidence for physics beyond the Standard Model, limits on the physical parameters of the models can be derived. There is no universally agreed way to compute limits, particularly when measurements lie close to a physical boundary. Where possible results of the interpretations are first quoted as measurements, and the methods used to derive limits from these results are discussed.

In principle the introduction of processes beyond the Standard Model will affect the cross-section for the channel $\mathrm{e}^{+} \mathrm{e}^{-} \rightarrow \mathrm{e}^{+} \mathrm{e}^{-}$at small angles, on which the measurement of the luminosity is based. However for values of the parameters of the models discussed in this section, the changes to the cross-section in this region are negligible compared with the experimental and theoretical uncertainties discussed in Section 4.

\footnotetext{
${ }^{4}$ In the comparison of the data with the models, it has been assumed that the one-loop electroweak corrections to the observables are those given by the Standard Model. It has been pointed out [33] that this may not always be the case.
} 


\subsection{S-matrix ansatz}

\subsubsection{Theory}

The S-matrix formalism [34] is a rigorous semi model-independent approach to describe the cross-sections and the forward-backward asymmetries in $\mathrm{e}^{+} \mathrm{e}^{-}$annihilations. In this model, the cross-sections can be parametrised as follows:

$$
\sigma_{\mathrm{a}}^{0}(s)=\frac{4}{3} \pi \alpha^{2}(\hbar c)^{2}\left[\frac{\mathrm{g}_{\mathrm{f}}^{\mathrm{a}}}{s}+\frac{\mathrm{j}_{\mathrm{f}}^{\mathrm{a}}\left(s-\overline{\mathrm{M}}_{\mathrm{Z}}{ }^{2} c^{4}\right)+\mathrm{r}_{\mathrm{f}}^{\mathrm{a}} s}{\left(s-\overline{\mathrm{M}}_{\mathrm{Z}}^{2} c^{4}\right)^{2}+\overline{\mathrm{M}}_{\mathrm{Z}}^{2} c^{4} \bar{\Gamma}_{\mathrm{Z}}^{2}}\right] \quad \text { with } \begin{aligned}
& \mathrm{a}=\text { tot }, \mathrm{fb} \\
& \mathrm{f}=\text { had, e, } \mu, \tau,
\end{aligned}
$$

while the forward-backward asymmetries are given by:

$$
A_{\mathrm{FB}}^{0}(s)=\frac{3}{4} \frac{\sigma_{\mathrm{fb}}^{0}(s)}{\sigma_{\mathrm{tot}}^{0}(s)} .
$$

The mass $\overline{\mathrm{M}}_{\mathrm{Z}}$ and width $\bar{\Gamma}_{\mathrm{Z}}$ of the $\mathrm{Z}$ in the S-matrix formalism are related to the values obtained from Standard Model fits, $\mathrm{M}_{\mathrm{Z}}$ and $\Gamma_{\mathrm{Z}}$, in which an $s$ dependent width term is included by

$$
\begin{gathered}
\mathrm{M}_{\mathrm{Z}} \equiv \overline{\mathrm{M}}_{\mathrm{Z}} \sqrt{1+\bar{\Gamma}_{\mathrm{Z}}^{2} / \overline{\mathrm{M}}_{\mathrm{Z}}^{2}} \approx \overline{\mathrm{M}}_{\mathrm{Z}}+34.20 \mathrm{MeV} / c^{2} \\
\Gamma_{\mathrm{Z}} \equiv \bar{\Gamma}_{\mathrm{Z}} \sqrt{1+\bar{\Gamma}_{\mathrm{Z}}^{2} / \overline{\mathrm{M}}_{\mathrm{Z}}^{2}} \approx \bar{\Gamma}_{\mathrm{Z}}+0.94 \mathrm{MeV}
\end{gathered}
$$

The parameters $\mathrm{r}_{\mathrm{f}}$ and $\mathrm{j}_{\mathrm{f}}$ respectively scale the $\mathrm{Z}$ exchange and the $\gamma \mathrm{Z}$ interference contributions to the total cross-section and forward-backward asymmetries; they are functions of the effective vector and axial-vector couplings of the fermions. The contribution $\mathrm{g}_{\mathrm{f}}$ of the pure $\gamma$ exchange was fixed to the value predicted by QED in all fits. The photonic virtual and bremsstrahlung corrections are included through the convolution of eqn. 15 with the photonic flux function.

\subsubsection{Results}

Published measurements from LEP I [1,2] and the runs above the $\mathrm{Z}$ in 1995-97 [3] and the results described in Section 5 were analysed in the framework of the S-matrix approach, achieving a substantial improvement in the precision of the $\gamma \mathrm{Z}$ interference compared to the accuracy obtained from the $\mathrm{Z}$ data alone $[1,2]$, and updating the results presented in reference [3].

Fits to the hadronic and leptonic cross-sections and leptonic forward-backward asymmetries were carried out in this framework using the corresponding branch of the ZFITTER/SMATASY $6.36[26,34,35]$ program. Data on $\mathrm{e}^{+} \mathrm{e}^{-} \rightarrow \mathrm{e}^{+} \mathrm{e}^{-}$at LEP II are not used in the fit due to the large $t$-channel contribution to the measurements, which is not described by the S-matrix formalism. In the fits, LEP I and LEP II measurements were assumed to be uncorrelated. Results for the mass and the width are quoted in terms of $\mathrm{M}_{\mathrm{Z}}$ and $\Gamma_{\mathrm{Z}}$.

The results of the fits are presented in Table 23. Using the LEP I data only, the $\chi^{2}$ amounted to 162.0 ( $n d o f=161$ ) for the 16-parameter fit (i.e. without assuming lepton universality), and to 176.1 ( $n d o f=169$ ) for the 8-parameter fit (where lepton universality was assumed), the number of fitted points being 177 . Using the combined LEP I and LEP II data, the $\chi^{2}$ amounted to 245.1 ( $\left.n d o f=221\right)$ and 256.1 ( $\left.n d o f=229\right)$ for the 16-parameter fit and the 8-parameter fit, respectively, the number of fitted points being 237. The correlation coefficients between the free parameters of the 16- and 8-parameter fits for the LEP I and LEP I + LEP II data are shown in Tables 24, 25, 26 and 27. 
The data support the hypothesis of lepton universality. Overall, the measurements are in good agreement with the Standard Model predictions.

The correlations between the parameters $\mathrm{M}_{\mathrm{Z}}$ and $\mathrm{j}_{\text {had }}^{\text {tot }}$ for 8-parameter fits to LEP I and LEP I + LEP II data are shown in Figure 13. It can be seen that a significant improvement on the precision on the hadronic interference parameter, $j_{\text {had }}^{\text {tot }}$, is obtained when the high energy data are included in the fit. The fitted value of $\mathrm{M}_{\mathrm{Z}}$ is consistent with the value obtained from a 5 parameter, Standard Model, fit to LEP I data [2], $\mathrm{M}_{\mathrm{Z}}=91.1863 \pm 0.0028 \mathrm{GeV} / c^{2}$, and the value of $\mathrm{j}_{\text {had }}^{\text {tot }}$ is consistent with, though somewhat above, the Standard Model expectation $\mathrm{j}_{\text {had }}^{\text {tot }}=0.2201_{-0.0039}^{+0.0024}$, where the uncertainties correspond to uncertainties in the input parameters.

\section{$6.2 \mathrm{Z}^{\prime}$ Bosons}

\subsubsection{Theory}

Many theories which are more general than the Standard Model predict the existence of additional heavy gauge bosons. The consequences of several of these models were investigated, complemented by a model-independent fit to the leptonic data.

The existence of an additional heavy gauge boson $\mathrm{Z}^{\prime}$ can be parametrised by the mass of the boson $\mathrm{M}_{\mathrm{z}^{\prime}}$ and by its couplings to fermions. In addition, a possible mixing between $Z^{\prime}$ and the standard $Z$, represented by a mixing angle $\Theta_{\mathrm{zz}^{\prime}}$, has to be taken into account $[36,37]$. In order to deal with a restricted number of free parameters, it is useful to consider specific $\mathrm{Z}^{\prime}$ models with well-defined couplings. Popular models are:

- The $E_{6}$ model $[38,39]$ is based on a symmetry breaking of the $E_{6}$ GUT where two gauge groups $U(1)_{\chi}$ and $U(1)_{\psi}$ are introduced,

$$
J_{\mathrm{z}^{\prime}}^{\mu}=J_{\chi}^{\mu} \cos \Theta_{6}+J_{\psi}^{\mu} \sin \Theta_{6}
$$

The free parameter, $\Theta_{6}$, of this model is the mixing of the $\chi$ and $\psi$ fields to form the $\mathrm{Z}^{\prime}$. Usual choices of $\Theta_{6}$ are $\Theta_{6}=0, \pi / 2,-\arctan \sqrt{5 / 3}(\chi, \psi$ and $\eta$ model $)$;

- The L-R model $[40,41]$ includes a right-handed $S U(2)_{R}$ extension to the Standard Model gauge group $S U(2)_{L} \otimes U(1)$. The free parameter $\alpha_{L R}$ describes the coupling of the heavy bosons to fermions. $\alpha_{L R}$ can take values between $\sqrt{2 / 3} \leq \alpha_{L R} \leq$ $\sqrt{\cot ^{2} \theta_{W}-1}(\sim 1.53)$, where $\theta_{W}$ is the weak mixing angle.

In a more general approach, the $\mathrm{Z}^{\prime}$ boson is directly described in terms of its couplings $a_{f}^{\prime}$ and $v_{f}^{\prime}$ [42]. Off the $\mathrm{Z}^{\prime}$ resonance, pair production is only sensitive to the normalised couplings $a_{f}^{N}$ and $v_{f}^{N}$. As a consequence, the couplings and the mass of the $\mathrm{Z}^{\prime}$ boson cannot be measured independently. The normalised couplings are:

$$
a_{f}^{N}=a_{f}^{\prime} \sqrt{\frac{s}{\mathrm{M}_{\mathrm{Z}^{\prime}}^{2}-s}}, \quad v_{f}^{N}=v_{f}^{\prime} \sqrt{\frac{s}{\mathrm{M}_{\mathrm{Z}^{\prime}}^{2}-\mathrm{s}}} .
$$

\subsubsection{Results}

The non-radiative hadronic total cross-sections and $\mu^{+} \mu^{-}$and $\tau^{+} \tau^{-}$total cross-sections and asymmetries presented here together with existing data from LEP I $[1,2]$ and LEP II at $\sqrt{s} \sim 130-172 \mathrm{GeV}[3]$ were used to fit the data to models including additional $\mathrm{Z}^{\prime}$ bosons. 


\begin{tabular}{|c|c|c|c|c|c|}
\hline & \multicolumn{2}{|c|}{ LEP I } & \multicolumn{2}{|c|}{ LEP I + LEP II } & $\mathrm{SM}$ \\
\hline $\mathrm{M}_{\mathrm{Z}}$ & $91.1936 \pm 0.0112$ & $91.1808 \pm 0.0094$ & $91.1844 \pm 0.0036$ & $91.1831 \pm 0.0034$ & - \\
\hline$\Gamma_{\mathrm{Z}}$ & $2.4861 \pm 0.0048$ & $2.4886 \pm 0.0046$ & $2.4894 \pm 0.0041$ & $2.4893 \pm 0.0041$ & 2.497 \\
\hline $\mathrm{r}_{\text {had }}^{\text {tot }}$ & $2.9490 \pm 0.0110$ & $2.9543 \pm 0.0107$ & $2.9567 \pm 0.0096$ & $2.9564 \pm 0.0095$ & 2.966 \\
\hline$r_{e}^{\text {tot }}$ & $0.14092 \pm 0.00095$ & & $0.14129 \pm 0.00091$ & & \\
\hline $\mathrm{r}_{\mu}^{\text {tot }}$ & $0.14274 \pm 0.00072$ & & $0.14301 \pm 0.00067$ & & \\
\hline $\mathrm{r}_{\tau}^{\text {tot }}$ & $0.14161 \pm 0.00100$ & & $0.14204 \pm 0.00096$ & & \\
\hline $\mathrm{r}_{1}^{\text {tot }}$ & & $0.14230 \pm 0.00062$ & & $0.14240 \pm 0.00058$ & 0.1427 \\
\hline jot & $-0.21 \pm 0.64$ & $0.51 \pm 0.55$ & $0.44 \pm 0.12$ & $0.47 \pm 0.12$ & 0.22 \\
\hline $\mathrm{je}_{\mathrm{e}}^{\text {tot }}$ & $-0.094 \pm 0.075$ & & $-0.042 \pm 0.048$ & & \\
\hline $\mathrm{j}_{\mu}^{\text {tot }}$ & $0.056 \pm 0.042$ & & $0.029 \pm 0.019$ & & \\
\hline $\mathbf{j}_{\tau}^{\text {tot }}$ & $0.004 \pm 0.046$ & & $-0.013 \pm 0.026$ & & \\
\hline $\mathrm{j}_{1}^{\text {tot }}$ & & $0.047 \pm 0.037$ & & $0.010 \pm 0.015$ & 0.004 \\
\hline $\mathrm{r}_{\mathrm{e}}^{\mathrm{fb}}$ & $0.00306 \pm 0.00091$ & & $0.00298 \pm 0.00090$ & & \\
\hline $\mathrm{r}_{\mu}^{\mathrm{fb}}$ & $0.00275 \pm 0.00051$ & & $0.00286 \pm 0.00049$ & & \\
\hline $\mathrm{r}_{\tau}^{\mathrm{fb}}$ & $0.00406 \pm 0.00072$ & & $0.00428 \pm 0.00070$ & & \\
\hline $\mathrm{r}_{1}^{\mathrm{fb}}$ & & $0.00304 \pm 0.00038$ & & $0.00327 \pm 0.00037$ & 0.00273 \\
\hline $\mathrm{je}_{\mathrm{e}}^{\mathrm{fb}}$ & $0.802 \pm 0.075$ & & $0.805 \pm 0.075$ & & \\
\hline $\mathrm{j}_{\mu}^{\mathrm{fb}}$ & $0.711 \pm 0.037$ & & $0.802 \pm 0.024$ & & \\
\hline $\mathrm{j}_{\tau}^{\mathrm{fb}}$ & $0.707 \pm 0.047$ & & $0.832 \pm 0.031$ & & \\
\hline $\mathrm{j}_{1}^{\mathrm{fb}}$ & & $0.725 \pm 0.027$ & & $0.811 \pm 0.018$ & 0.799 \\
\hline
\end{tabular}

Table 23: Results of the 16- and 8-parameter fits to the LEP I only and combined LEP $I+L E P I I$ data. Also shown are the Standard Model predictions for the fit parameters. $\mathrm{M}_{\mathrm{Z}}\left(\Gamma_{\mathrm{Z}}\right)$ are measured in units of $\mathrm{GeV} / \mathrm{c}^{2}(\mathrm{GeV})$, all other quantities are dimensionless. 


\begin{tabular}{|c|c|c|c|c|c|c|c|c|c|c|c|c|c|c|c|}
\hline & $\Gamma_{\mathrm{z}}$ & $\mathrm{r}_{\text {had }}^{\text {tot }}$ & $\mathrm{r}_{\mathrm{e}}^{\mathrm{tot}}$ & $\mathrm{r}_{\mu}^{\mathrm{tot}}$ & $\mathrm{r}_{\tau}^{\mathrm{tot}}$ & $\mathrm{j}_{\text {had }}^{\text {tot }}$ & $\mathrm{j}_{\mathrm{e}}^{\text {tot }}$ & $\mathrm{j}_{\mu}^{\text {tot }}$ & $\mathrm{j}_{\tau}^{\text {tot }}$ & $\mathrm{r}_{\mathrm{e}}^{\mathrm{fb}}$ & $\mathrm{r}_{\mu}^{\mathrm{fb}}$ & $\mathrm{r}_{\tau}^{\mathrm{fb}}$ & $\mathrm{j}_{\mathrm{e}}^{\mathrm{fb}}$ & $\mathrm{j}_{\mu}^{\mathrm{fb}}$ & $\mathrm{j}_{\tau}^{\mathrm{fb}}$ \\
\hline $\mathrm{M}_{\mathrm{Z}}$ & -0.50 & -0.46 & -0.29 & -0.32 & -0.25 & -0.96 & -0.80 & -0.70 & -0.64 & 0.13 & 0.24 & 0.16 & -0.03 & 0.00 & 0.00 \\
\hline$\Gamma_{\mathrm{Z}}$ & & 0.90 & 0.52 & 0.67 & 0.49 & 0.53 & 0.40 & 0.38 & 0.35 & -0.06 & -0.11 & -0.07 & 0.04 & 0.04 & 0.03 \\
\hline $\mathrm{r}_{\text {had }}^{\text {tot }}$ & & & 0.53 & 0.68 & 0.49 & 0.49 & 0.37 & 0.35 & 0.32 & -0.05 & -0.10 & -0.06 & 0.04 & 0.05 & 0.04 \\
\hline$r_{e}^{\text {tot }}$ & & & & 0.39 & 0.28 & 0.30 & 0.26 & 0.22 & 0.20 & 0.07 & -0.06 & -0.04 & 0.08 & 0.03 & 0.02 \\
\hline $\mathrm{r}_{\mu}^{\mathrm{tot}}$ & & & & & 0.36 & 0.34 & 0.26 & 0.33 & 0.23 & -0.03 & -0.05 & -0.04 & 0.03 & 0.08 & 0.03 \\
\hline $\mathrm{r}_{\tau}^{\mathrm{tot}}$ & & & & & & 0.26 & 0.20 & 0.19 & 0.25 & -0.03 & -0.05 & 0.00 & 0.02 & 0.02 & 0.09 \\
\hline $\mathrm{j}_{\text {had }}^{\text {tot }}$ & & & & & & & 0.78 & 0.70 & 0.63 & -0.12 & -0.24 & -0.16 & 0.03 & 0.00 & 0.01 \\
\hline $\mathrm{j}_{\mathrm{e}}^{\text {tot }}$ & & & & & & & & 0.57 & 0.52 & -0.08 & -0.20 & -0.13 & 0.10 & 0.00 & 0.00 \\
\hline $\mathrm{j}_{\mu}^{\mathrm{tot}}$ & & & & & & & & & 0.46 & -0.09 & -0.15 & -0.12 & 0.02 & -0.04 & 0.00 \\
\hline $\mathrm{j}_{\tau}^{\text {tot }}$ & & & & & & & & & & -0.08 & -0.16 & -0.08 & 0.02 & 0.00 & -0.04 \\
\hline $\mathrm{r}_{\mathrm{e}}^{\mathrm{fb}}$ & & & & & & & & & & & 0.04 & 0.03 & 0.09 & 0.00 & 0.00 \\
\hline $\mathrm{r}_{\mu}^{\mathrm{fb}}$ & & & & & & & & & & & & 0.05 & -0.01 & 0.20 & 0.00 \\
\hline $\mathrm{r}_{\tau}^{\mathrm{fb}}$ & & & & & & & & & & & & & 0.00 & 0.00 & 0.18 \\
\hline $\mathrm{j}_{\mathrm{e}}^{\mathrm{fb}}$ & & & & & & & & & & & & & & 0.00 & 0.00 \\
\hline $\mathrm{j}_{\mu}^{\mathrm{fb}}$ & & & & & & & & & & & & & & & 0.00 \\
\hline
\end{tabular}




\begin{tabular}{|c|c|c|c|c|c|c|c|c|c|c|c|c|c|c|c|}
\hline & $\Gamma_{\mathrm{Z}}$ & $\mathrm{r}_{\text {had }}^{\text {tot }}$ & $\mathrm{r}_{\mathrm{e}}^{\mathrm{tot}}$ & $\mathrm{r}_{\mu}^{\mathrm{tot}}$ & $\mathrm{r}_{\tau}^{\text {tot }}$ & $\mathrm{j}_{\text {had }}^{\text {tot }}$ & $\mathrm{j}_{\mathrm{e}}^{\text {tot }}$ & $\mathrm{j}_{\mu}^{\mathrm{tot}}$ & $\mathrm{j}_{\tau}^{\text {tot }}$ & $\mathrm{r}_{\mathrm{e}}^{\mathrm{fb}}$ & $\mathrm{r}_{\mu}^{\mathrm{fb}}$ & $\mathrm{r}_{\tau}^{\mathrm{fb}}$ & $\mathrm{j}_{\mathrm{e}}^{\mathrm{fb}}$ & $\mathrm{j}_{\mu}^{\mathrm{fb}}$ & $\mathrm{j}_{\tau}^{\mathrm{fb}}$ \\
\hline $\mathrm{M}_{\mathrm{Z}}$ & 0.03 & 0.06 & 0.01 & 0.04 & 0.02 & -0.55 & -0.35 & -0.15 & -0.16 & 0.04 & 0.08 & 0.05 & 0.00 & -0.05 & -0.06 \\
\hline$\Gamma_{\mathrm{Z}}$ & & 0.87 & 0.44 & 0.61 & 0.42 & 0.02 & -0.02 & 0.02 & 0.02 & 0.01 & 0.02 & 0.02 & 0.03 & 0.05 & 0.05 \\
\hline $\mathrm{r}_{\text {had }}^{\text {tot }}$ & & & 0.45 & 0.62 & 0.43 & 0.00 & -0.03 & 0.01 & 0.02 & 0.01 & 0.02 & 0.02 & 0.03 & 0.05 & 0.04 \\
\hline $\mathrm{r}_{\mathrm{e}}^{\mathrm{tot}}$ & & & & 0.31 & 0.22 & 0.01 & 0.04 & 0.01 & 0.01 & 0.12 & 0.01 & 0.01 & 0.07 & 0.03 & 0.02 \\
\hline $\mathrm{r}_{\mu}^{\text {tot }}$ & & & & & 0.30 & -0.01 & -0.02 & 0.08 & 0.01 & 0.01 & 0.03 & 0.02 & 0.02 & 0.10 & 0.03 \\
\hline $\mathrm{r}_{\tau}^{\mathrm{tot}}$ & & & & & & 0.00 & -0.01 & 0.01 & 0.09 & 0.01 & 0.01 & 0.04 & 0.01 & 0.03 & 0.13 \\
\hline $\mathrm{j}_{\text {had }}^{\text {tot }}$ & & & & & & & 0.23 & 0.12 & 0.14 & -0.02 & -0.05 & -0.03 & 0.00 & 0.05 & 0.06 \\
\hline $\mathrm{j}_{\mathrm{e}}^{\mathrm{tot}}$ & & & & & & & & 0.06 & 0.06 & 0.03 & -0.03 & -0.02 & 0.12 & 0.02 & 0.03 \\
\hline $\mathrm{j}_{\mu}^{\text {tot }}$ & & & & & & & & & 0.04 & -0.01 & 0.07 & -0.01 & 0.00 & 0.34 & 0.02 \\
\hline $\mathrm{j}_{\tau}^{\text {tot }}$ & & & & & & & & & & & & 0.08 & 0.00 & 0.02 & 0.39 \\
\hline $\mathrm{r}_{\mathrm{e}}^{\mathrm{fb}}$ & & & & & & & & & & & 0.01 & 0.01 & 0.09 & 0.00 & 0.00 \\
\hline $\mathrm{r}_{\mu}^{\mathrm{fb}}$ & & & & & & & & & & & & 0.02 & 0.00 & 0.12 & 0.00 \\
\hline $\mathrm{r}_{\tau}^{\mathrm{fb}}$ & & & & & & & & & & & & & 0.00 & 0.00 & 0.11 \\
\hline $\mathrm{j}_{\mathrm{e}}^{\mathrm{fb}}$ & & & & & & & & & & & & & & 0.00 & 0.00 \\
\hline $\mathrm{j}_{\mu}^{\mathrm{fb}}$ & & & & & & & & & & & & & & & 0.01 \\
\hline
\end{tabular}




\begin{tabular}{|l||c|c|c|c|c|c|c|}
\hline & $\Gamma_{\mathrm{Z}}$ & $\mathrm{r}_{\text {had }}^{\text {tot }}$ & $\mathrm{r}_{1}^{\text {tot }}$ & $\mathrm{j}_{\text {hat }}^{\text {tot }}$ & $\mathrm{j}_{1}^{\text {tot }}$ & $\mathrm{r}_{1}^{\mathrm{fb}}$ & $\mathrm{j}_{\mathrm{l}}^{\mathrm{fb}}$ \\
\hline \hline $\mathrm{M}_{\mathrm{Z}}$ & -0.42 & -0.39 & -0.32 & -0.95 & -0.83 & 0.27 & 0.03 \\
$\Gamma_{\mathrm{Z}}$ & & 0.90 & 0.74 & 0.46 & 0.38 & -0.09 & 0.05 \\
$\mathrm{r}_{\text {had }}^{\text {tot }}$ & & & 0.75 & 0.43 & 0.35 & -0.08 & 0.05 \\
$\mathrm{r}_{1}^{\text {tot }}$ & & & & 0.35 & 0.33 & -0.04 & 0.09 \\
$\mathrm{j}_{\text {hat }}^{\text {tot }}$ & & & & & 0.81 & -0.26 & -0.03 \\
$\mathrm{j}_{1}^{\text {tot }}$ & & & & & & -0.20 & -0.04 \\
$\mathrm{r}_{1}^{\mathrm{fb}}$ & & & & & & & 0.17 \\
\hline
\end{tabular}

Table 26: Correlation matrix for the 8-parameter fit to LEP I data.

\begin{tabular}{|l||c|c|c|c|c|c|c|}
\hline & $\Gamma_{\mathrm{Z}}$ & $\mathrm{r}_{\text {had }}^{\text {tot }}$ & $\mathrm{r}_{1}^{\text {tot }}$ & $\mathrm{j}_{\text {had }}^{\text {tot }}$ & $\mathrm{j}_{1}^{\text {tot }}$ & $\mathrm{r}_{1}^{\mathrm{fb}}$ & $\mathrm{j}_{1}^{\mathrm{fb}}$ \\
\hline \hline $\mathrm{M}_{\mathrm{Z}}$ & 0.02 & 0.05 & 0.03 & -0.53 & -0.29 & 0.09 & -0.10 \\
$\Gamma_{\mathrm{Z}}$ & & 0.87 & 0.70 & 0.03 & 0.02 & 0.03 & 0.07 \\
$\mathrm{r}_{\text {had }}^{\text {tot }}$ & & & 0.71 & 0.01 & 0.01 & 0.03 & 0.07 \\
$\mathrm{r}_{\mathrm{l}}^{\text {tot }}$ & & & 0.00 & 0.07 & 0.06 & 0.12 \\
$\mathrm{j}_{\text {had }}^{\text {tot }}$ & & & & & 0.22 & -0.06 & 0.08 \\
$\mathrm{j}_{1}^{\text {tot }}$ & & & & & & 0.05 & 0.35 \\
$\mathrm{r}_{1}^{\mathrm{fb}}$ & & & & & & 0.10 \\
\hline
\end{tabular}

Table 27: Correlation matrix for the 8-parameter fit to LEP I + LEP II data. 


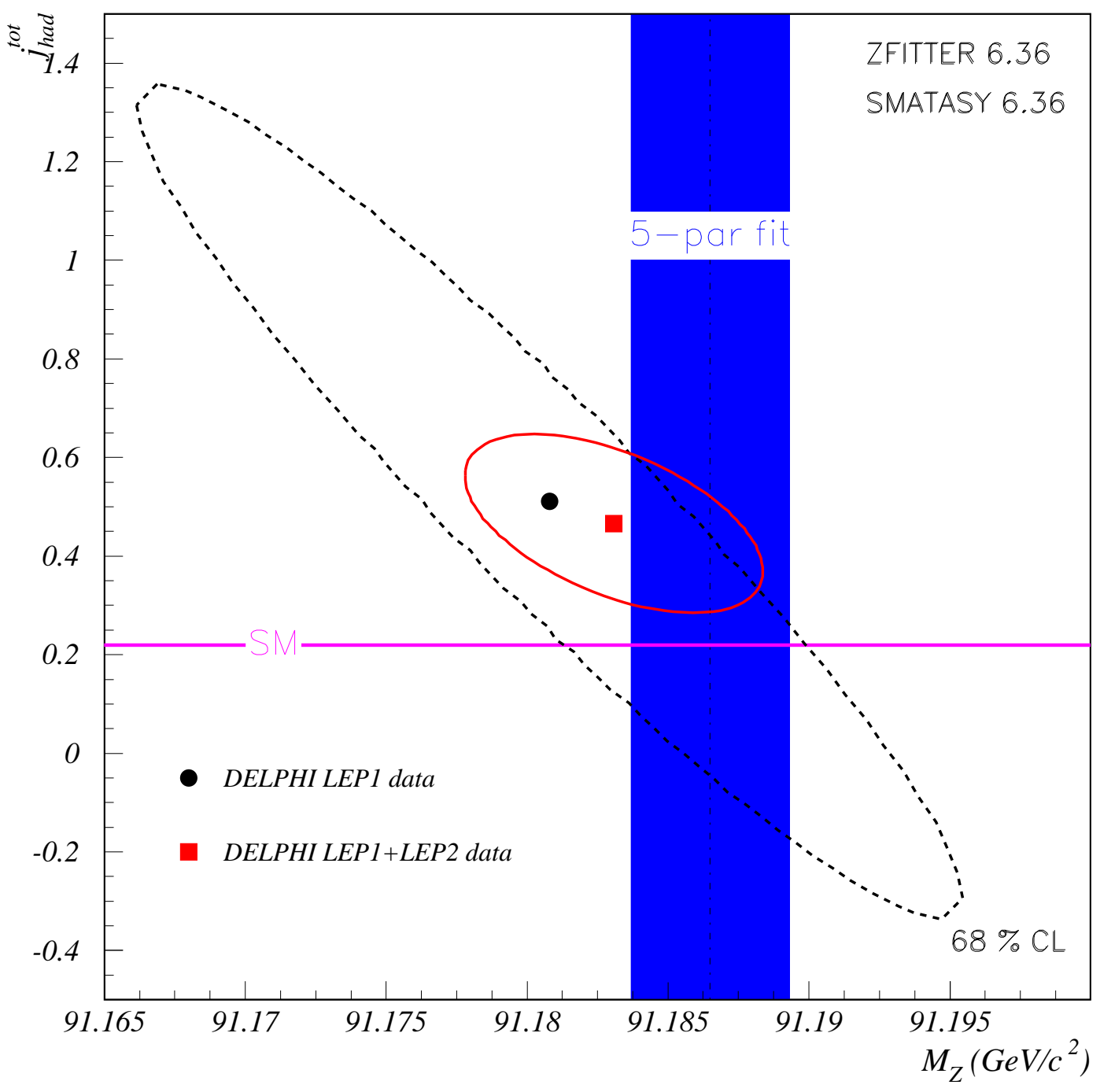

Figure 13: Contour plot in the $\left(\mathrm{M}_{\mathrm{Z}}, \mathrm{j}\right.$ had $)$ plane. The dashed curve shows the region accepted at the 68\% confidence level from an 8-parameter fit to data taken at the energies around the $\mathrm{Z}$; the solid curve shows the region accepted at the same confidence level when the high energy data are also included in the fit. The band labelled 5-par fit is the fit to $\mathrm{M}_{\mathrm{Z}}$ obtained from a 5-parameter, Standard Model, fit to LEP I data. The narrow band labelled SM is the Standard Model expectation for $\mathrm{j}_{\mathrm{had}}^{\text {tot }}$, as calculated using ZFITTER. 
Fits were made to the mass of $\mathrm{Z}^{\prime}, \mathrm{M}_{\mathrm{Z}^{\prime}}$, the mass of the $\mathrm{Z}, \mathrm{M}_{\mathrm{Z}}$, and to the mixing angle between the two bosonic fields, $\Theta_{\mathrm{zz}^{\prime}}$, for 4 different models referred to as $\chi, \psi, \eta$ and L-R as described in 6.2.1. The theoretical predictions came from the ZEFIT package (version 6.10) [36] together with the ZFITTER program (version 6.10). The program ZEFIT provides predictions for the cross-sections and forward-backward asymmetries for each model as a function of $\mathrm{M}_{\mathrm{Z}}, \mathrm{M}_{\mathrm{z}^{\prime}}, \Theta_{\mathrm{zz}}$, the masses of the Higgs boson, $\mathrm{M}_{\mathrm{H}}$, and the top quark, $\mathrm{m}_{\mathrm{t}}$, the strong coupling constant, $\alpha_{s}$, and the $\mathrm{Z}^{\prime}$-model parameters $\Theta_{6}$ or $\alpha_{L R}$. For the L-R model $\alpha_{L R}$ was set to 1.1. In order to reduce the number of free parameters, the following input parameters were used: $\mathrm{m}_{\mathrm{t}}=175 \mathrm{GeV} / c^{2}, \mathrm{M}_{\mathrm{H}}=150 \mathrm{GeV} / c^{2}, \alpha_{s}=$ 0.118 .

The correlations between the experimental errors were taken into account when the $\chi^{2}$ was calculated between the predictions and the measurements. The most important correlation is between the errors on the luminosity for the cross-section measurements. Correlations between LEP I and LEP II measurements are very small and therefore neglected.

No evidence was found for the existence of a $\mathrm{Z}^{\prime}$-boson in any of the models. The fitted value of $\mathrm{M}_{\mathrm{Z}}$ was found to be in agreement with the Standard Model value. Twodimensional exclusion contours at $95 \%$ confidence level, obtained with $\chi^{2}>\chi_{\min }^{2}+$ 5.99 [43] in the $\mathrm{M}_{\mathrm{z}^{\prime}}-\Theta_{\mathrm{zz}}$ plane, were made. The allowed regions for $\mathrm{M}_{\mathrm{z}^{\prime}}$ and $\Theta_{\mathrm{zz}^{\prime}}$ are shown in Figure 14. The one-dimensional limits at $95 \%$ confidence level for both $\mathrm{M}_{\mathrm{Z}^{\prime}}$ and $\Theta_{\mathrm{zz}^{\prime}}$, obtained with $\chi^{2}>\chi_{\min }^{2}+3.84$, are shown in Table 28. We repeat that these limits are based on the assumption that the electroweak one-loop corrections are those of the SM. In particular for the L-R symmetric model, the authors of reference [33] have shown that inclusion of the extra gauge bosons in the corrections can affect the limits on the mixing angle. The limits for the $\mathrm{Z}^{\prime}$ mass range from 360 to $545 \mathrm{GeV} / c^{2}$, an increase of between 50 and $125 \mathrm{GeV} / c^{2}$ on the limits presented in [4], depending on the model.

In addition the Sequential Standard Model [44] has been considered. This model proposes the existence of a $\mathrm{Z}^{\prime}$ with exactly the same coupling to fermions as the Standard Model Z. A limit of $\mathrm{M}_{\mathrm{Z}^{\prime}}>1305 \mathrm{GeV} / c^{2}$ is found at $95 \%$ confidence level, an increase of $595 \mathrm{GeV} / c^{2}$ on the limit presented in [4].

Model-independent fits were performed to the leptonic cross-sections and forwardbackward asymmetries, for the leptonic couplings of a $\mathrm{Z}^{\prime}, a_{l^{\prime}}^{N}$ and $v_{l^{\prime}}^{N}$, normalised for the overall coupling scale and the mass of the $\mathrm{Z}^{\prime}[42]$.

Several values of the mass of the $Z^{\prime}$ were considered (i.e. 300,500 and $1000 \mathrm{GeV} / c^{2}$ ), and the $\mathrm{ZZ}^{\prime}$-mixing was neglected. The $95 \%$ confidence level limits on the normalised couplings are $\left|a_{l^{\prime}}^{N}\right|<0.19$ and $\left|v_{l^{\prime}}^{N}\right|<0.19$, an increase of 0.04 and a decrease of 0.03 , respectively, on limits given in [4].

\subsection{Contact interactions}

\subsubsection{Theory}

Contact interactions between initial and final state fermionic currents provide a rather general description of the low energy behaviour of new physics with a characteristic high energy scale. Following reference [45], these interactions are parameterised by an effective Lagrangian, $\mathcal{L}_{e f f}$, of the form:

$$
\mathcal{L}_{e f f}=\frac{g^{2}}{(1+\delta) \Lambda^{2}} \sum_{i, j=L, R} \eta_{i j} \bar{e}_{i} \gamma_{\mu} e_{i} \bar{f}_{j} \gamma^{\mu} f_{j},
$$




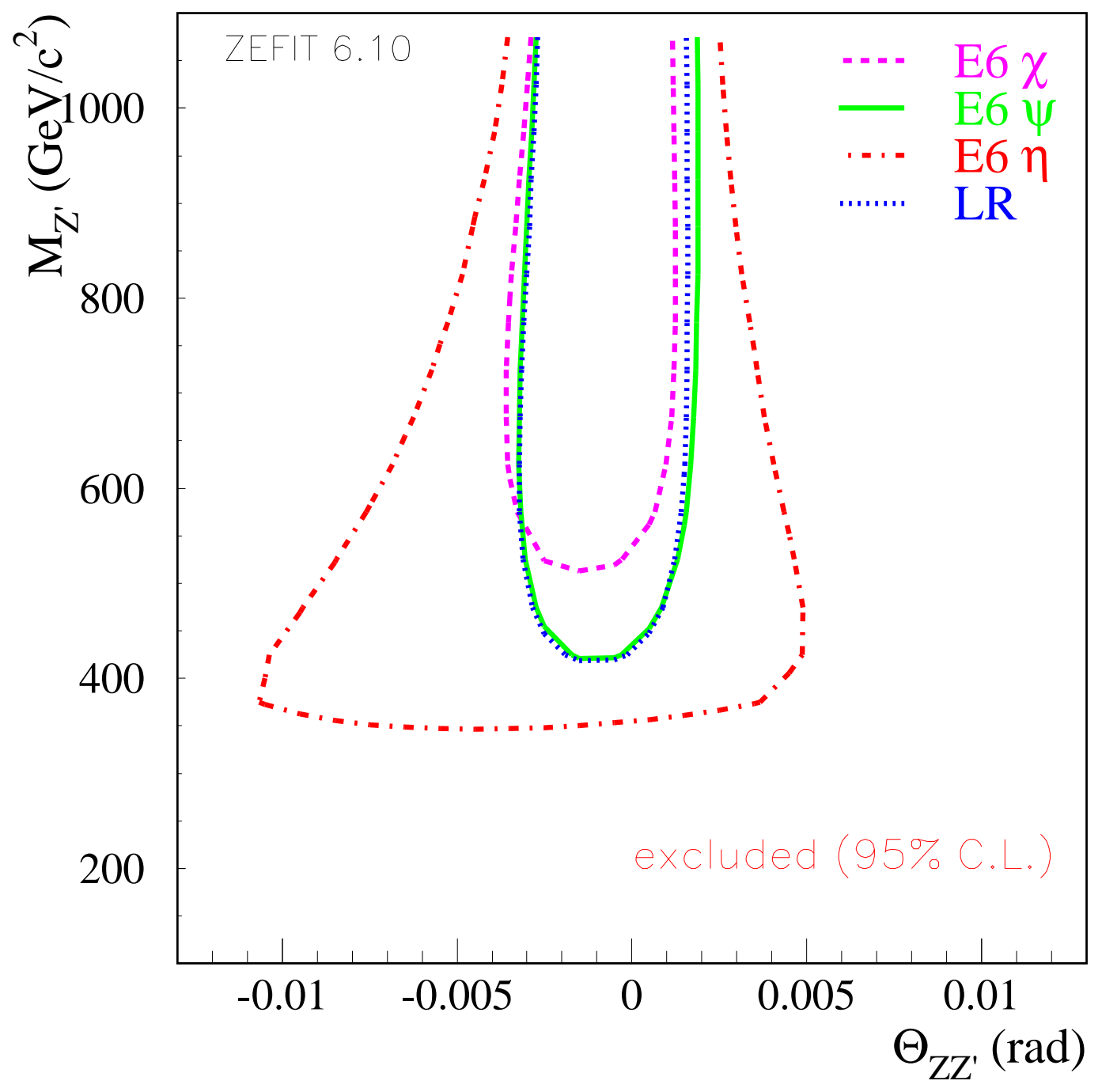

Figure 14: The allowed domains in the $\Theta_{\mathrm{zz}^{\prime}}-\mathrm{M}_{\mathrm{z}^{\prime}}$ plane for the $\chi, \psi, \eta$ and $L-R$ models. Values outside the contours are excluded at 95\% confidence level.

\begin{tabular}{|c|c|c|c|c|}
\hline Model & $\chi$ & $\psi$ & $\eta$ & L-R \\
\hline $\mathrm{M}_{\mathrm{Z}^{\prime}}^{\operatorname{limit}}\left(\mathrm{GeV} / c^{2}\right)$ & 545 & 475 & 360 & 455 \\
\hline$\Theta_{\mathrm{ZZ}^{\prime}}^{\text {limit }} \mid \quad(\mathrm{mrad})$ & 3.1 & 2.7 & 9.2 & 2.8 \\
\hline
\end{tabular}

Table 28: $95 \%$ confidence level lower limits on the $\mathrm{Z}^{\prime}$ mass and upper limits on the ZZ' mixing angle within the $\chi, \psi, \eta$ and $L-R$ models. 


\begin{tabular}{|c|c|c|c|c|}
\hline Model & $\eta_{L L}$ & $\eta_{R R}$ & $\eta_{L R}$ & $\eta_{R L}$ \\
\hline \hline $\mathrm{LL}^{ \pm}$ & \pm 1 & 0 & 0 & 0 \\
\hline $\mathrm{RR}^{ \pm}$ & 0 & \pm 1 & 0 & 0 \\
\hline $\mathrm{VV}^{ \pm}$ & \pm 1 & \pm 1 & \pm 1 & \pm 1 \\
\hline $\mathrm{AA}^{ \pm}$ & \pm 1 & \pm 1 & $\mp 1$ & $\mp 1$ \\
\hline $\mathrm{LR}^{ \pm}$ & 0 & 0 & \pm 1 & 0 \\
\hline $\mathrm{RL}^{ \pm}$ & 0 & 0 & 0 & \pm 1 \\
\hline $\mathrm{V}^{ \pm}$ & \pm 1 & \pm 1 & 0 & 0 \\
\hline $\mathrm{A}^{ \pm}$ & 0 & 0 & \pm 1 & \pm 1 \\
\hline
\end{tabular}

Table 29: Choices of $\eta_{i j}$ for different contact interaction models, LL, RR etc. 
where $g^{2} / 4 \pi$ is taken to be 1 by convention, $\delta=1(0)$ for $f=e(f \neq e), \eta_{i j}= \pm 1$ or $0, \Lambda$ is the scale of the contact interactions ${ }^{5}, e_{i}$ and $f_{j}$ are left or right-handed spinors. This effective Lagrangian is added to the Standard Model Lagrangian. By assuming different helicity couplings between the initial-state and final-state currents and either constructive or destructive interference with the Standard Model (according to the choice of each $\eta_{i j}$ ) a set of different models can be defined from this Lagrangian [46]. The values of $\eta_{i j}$ for the models investigated in this paper are given in Table 29. The differential cross-section for scattering the outgoing fermion at an angle $\theta$ with respect to the incident $e^{-}$direction is given by $[47,48]$

$$
\mathrm{d} \sigma / \mathrm{d} \cos \theta=\frac{\pi \alpha^{2}}{2 s} N_{c}^{f}\left\{\begin{array}{c}
{\left[\left|\bar{A}_{L R}^{e e}\right|^{2}+\left|\bar{A}_{R L}^{e e}\right|^{2}\right]\left(\frac{s}{t}\right)^{2} \delta+} \\
{\left[\left|A_{L R}^{e f}\right|^{2}+\left|A_{R L}^{e f}\right|^{2}\right]\left(\frac{t}{s}\right)^{2}+} \\
{\left[\left|A_{L L}^{e f}\right|^{2}+\left|A_{R R}^{e f}\right|^{2}\right]\left(\frac{u}{s}\right)^{2}}
\end{array}\right\},
$$

where $s, t$ and $u$ are the Mandelstam variables and $N_{c}^{f}$ is the number of colours for fermion $f$. The $A_{i j}$ and $\bar{A}_{i j}$ are helicity amplitudes for the scattering process [45]. When the helicity amplitudes are squared, 3 sets of terms arise: the first set contains purely Standard Model terms; the second set of terms derive from the interference between contact interactions and the Standard Model, these terms are proportional to $1 / \Lambda^{2}$; the final set of terms are due to contact interactions alone and are proportional to $1 / \Lambda^{4}$. For the purpose of fitting contact interaction models to the data, a new parameter $\epsilon=1 / \Lambda^{2}$ is defined, with $\epsilon=0$ in the limit that there are no contact interactions. This parameter is allowed to take both positive and negative values in the fits. It is worth noting that there is a symmetry between models with $\eta_{i j}=+1$ and those with $\eta_{i j}=-1$. The predicted differential cross-section in the constructive $(+)$ models is the same as the destructive $(-)$ models for $\epsilon^{-}=-\epsilon^{+}$. Therefore, starting from a model with constructive interference with the Standard Model, the region $\epsilon \geq 0$ represents physical values of $1 / \Lambda^{2}$, while the region $\epsilon \leq 0$ represents physical values for the equivalent model with destructive interference.

\subsubsection{Results}

Cross-section and forward-backward asymmetry measurements from all LEP II centreof-mass energies were compared to each of the contact interaction models mentioned above ${ }^{6}$ using $\chi^{2}$ fits, considering separately the $e^{+} e^{-}, \mu^{+} \mu^{-}$and $\tau^{+} \tau^{-}$final states, and a simultaneous fit to all three final states, assuming lepton universality in the contact interactions to obtain $\chi^{2}$ curves as a function of $\epsilon$. The predicted tree-level results were corrected for QED radiation. Correlations between the systematic uncertainties were taken into account in the fits. Making use of the symmetry, mentioned above, between models with constructive and destructive interference with the Standard Model, it is possible to fit pairs of models by allowing $\epsilon$ to take both positive and negative values.

The resulting $\chi^{2}$ as a function of $\epsilon$ were not always Gaussian-like parabolae. The values of $\epsilon$ extracted from the points with minimum $\chi^{2}$ from each of the fits were all compatible with the Standard Model expectation $\epsilon=0 \mathrm{TeV}^{-2}$, i.e. the differences in $\chi^{2}$ between the best fit points and the Standard Model point were always less than nine,

\footnotetext{
${ }^{5}$ The choice of $g^{2}$ is somewhat arbitrary; if the coupling constant was taken to be $\alpha_{s}$ much lower limits on $\Lambda$ would be obtained.

${ }^{6}$ For leptonic final states, models with only $\eta_{L R}= \pm 1$ are equivalent to models with only $\eta_{R L}= \pm 1$.
} 
except in the fit for the A0 model in $\mathrm{e}^{+} \mathrm{e}^{-} \rightarrow \tau^{+} \tau^{-}$, in which case the difference in $\chi^{2}$ was found to be 11.2.

Errors on $\epsilon$ were derived by finding the points above and below the best fit points for which the $\chi^{2}$ increased by nine above the minimum value, which would correspond to a three sigma uncertainty for Gaussian-like parabolae. These three sigma uncertainties were divided by three, to give one sigma errors. 95\% confidence level lower limits on $\Lambda$, by integrating under the likelihood curves, $\mathcal{L}(\epsilon)$, were obtained from the $\chi^{2}$ fits using

$$
\mathcal{L}(\epsilon)=\exp -\frac{1}{2} \chi^{2}(\epsilon)
$$

over the physically allowed region of $\epsilon$ for each choice of model ${ }^{7}$. The fitted values of $\epsilon$ with upper and lower errors and the limits on $\Lambda$ for models with constructive, $\Lambda^{+}$, and destructive, $\Lambda^{-}$, interference with the Standard Model are given in Table 30.

\subsection{Gravity in large extra dimensions}

\subsubsection{Theory}

The large difference between the electroweak scale $\left(\mathrm{M}_{\mathrm{EW}} \sim 10^{2}-10^{3} \mathrm{GeV}\right)$ and the scale at which quantum gravitational effects become strong, the Planck scale $\left(\mathrm{M}_{\mathrm{Pl}} \sim\right.$ $10^{19} \mathrm{GeV}$ ), leads to the well known "hierarchy problem". A solution, not relying on supersymmetry or technicolour, that involves an effective Planck scale, $\mathrm{M}_{\mathrm{D}}$, of $\mathcal{O}(\mathrm{TeV})$ is achieved by introducing $n$ compactified dimensions, into which spin 2 gravitons propagate, in addition to the 4 dimensions of standard space-time [49]. The Planck mass seen in the 4 uncompactified dimensions, $\mathrm{M}_{\mathrm{Pl}}$, can be expressed in terms of $\mathrm{M}_{\mathrm{D}}$, the effective Planck scale in the $n+4$ dimensional theory,

$$
\mathrm{M}_{\mathrm{Pl}}^{2} \sim \mathrm{R}^{n} \mathrm{M}_{\mathrm{D}}^{n+2}
$$

where $\mathrm{R}$ is the size of the extra dimensions. With $\mathrm{M}_{\mathrm{D}}=1 \mathrm{TeV}$, the case where $n=1$ is excluded as Newtonian gravitation would be modified at solar system distances, whereas $n=2$ corresponds to a radius for extra dimensions of $\mathcal{O}(1 \mathrm{~mm})$, which is excluded by recent gravitational experiments which test the inverse square law of gravitational attraction down to $\mathcal{O}(100 \mu \mathrm{m})$ [50]. There are also severe limits from astrophysics for a small number $(n=2,3)$ of large extra dimensions, however, higher numbers of dimensions are not ruled out. In addition it is possible to construct models which evade gravity and astrophysics bounds with a slight modification of the extra dimension scenario [51].

In high energy collisions at LEP and other colliders, new channels not present in the Standard Model would be available in which gravitons could be produced or exchanged. Virtual graviton exchange would affect the differential cross-section for $\mathrm{e}^{+} \mathrm{e}^{-} \rightarrow \mathrm{f} \overline{\mathrm{f}}$, with the largest contributions seen at low angles with respect to the incoming electron or positron. Embedding the model into a string model, and identifying the effective Planck scale, $\mathrm{M}_{\mathrm{D}}$, with the string scale, $\mathrm{M}_{\mathrm{s}}$, the differential cross-section for $\mathrm{e}^{+} \mathrm{e}^{-} \rightarrow \mathrm{f} \overline{\mathrm{f}}$ with the inclusion of the spin 2 graviton can be expressed as [52]

$$
\mathrm{d} \sigma / \mathrm{d} \cos \theta=\mathrm{d} \sigma /\left.\mathrm{d} \cos \theta\right|_{S M}+C_{1}^{f}(s, \cos \theta)\left[\frac{\lambda}{\mathrm{M}_{\mathrm{s}}^{4}}\right]+C_{2}^{f}(s, \cos \theta)\left[\frac{\lambda}{\mathrm{M}_{\mathrm{s}}^{4}}\right]^{2},
$$

\footnotetext{
${ }^{7}$ Integrating under the likelihood curve for $\epsilon$ is equivalent to obtaining a Bayesian limit assuming a prior uniform in $\epsilon$ over the physically allowed regions and zero for the unphysical regions.
} 


\begin{tabular}{|c|c|c|c|}
\hline \multicolumn{4}{|c|}{$e^{+} e^{-} \rightarrow e^{+} e^{-}$} \\
\hline Model & $\epsilon_{-\sigma_{-}}^{+\sigma_{+}}\left(\mathrm{TeV}^{-2}\right)$ & $\Lambda^{-}(\mathrm{TeV})$ & $\Lambda^{+}(\mathrm{TeV})$ \\
\hline \hline LL & $0.0071_{-0.0135}^{+0.0166}$ & 6.8 & 5.3 \\
\hline RR & $0.0106_{-0.0148}^{+0.0157}$ & 6.8 & 5.2 \\
\hline VV & $0.0024_{-0.0038}^{+0.0033}$ & 13.9 & 11.7 \\
\hline AA & $0.0176_{-0.0292}^{+0.0148}$ & 4.6 & 4.8 \\
\hline RL & $0.0035_{-0.0133}^{+0.0156}$ & 6.7 & 5.7 \\
\hline LR & $0.0035_{-0.0133}^{+0.0156}$ & 6.7 & 5.7 \\
\hline V0 & $0.0038_{-0.0069}^{+0.0077}$ & 10.1 & 8.0 \\
\hline A0 & $0.0058_{-0.0080}^{+0.0060}$ & 9.8 & 8.5 \\
\hline
\end{tabular}

\begin{tabular}{|c|c|c|c|}
\hline \multicolumn{4}{|c|}{$e^{+} \boldsymbol{e}^{-} \rightarrow \boldsymbol{\mu}^{+} \boldsymbol{\mu}^{-}$} \\
\hline Model & $\epsilon_{-\sigma_{-}}^{+\sigma_{+}}\left(\mathrm{TeV}^{-2}\right)$ & $\Lambda^{-}(\mathrm{TeV})$ & $\Lambda^{+}(\mathrm{TeV})$ \\
\hline \hline LL & $0.0019_{-0.0100}^{+0.0093}$ & 7.6 & 7.3 \\
\hline RR & $0.0016_{-0.0109}^{+0.0103}$ & 7.2 & 7.0 \\
\hline VV & $-0.0006_{-0.0034}^{+0.0040}$ & 12.9 & 12.2 \\
\hline AA & $0.0028_{-0.0057}^{+0.0045}$ & 10.9 & 10.1 \\
\hline RL & $-0.2377_{-0.0139}^{+0.0919}$ & 2.0 & 6.3 \\
\hline LR & $-0.2377_{-0.0139}^{+0.0919}$ & 2.0 & 6.3 \\
\hline V0 & $-0.0011_{-0.0044}^{+0.0057}$ & 11.5 & 10.9 \\
\hline A0 & $-0.2396_{-0.0067}^{+0.0866}$ & 2.0 & 9.0 \\
\hline
\end{tabular}

\begin{tabular}{|c|c|c|c|}
\hline \multicolumn{4}{|c|}{$e^{+} e^{-} \rightarrow \tau^{+} \boldsymbol{\tau}^{-}$} \\
\hline Model & $\epsilon_{-\sigma_{-}}^{+\sigma_{+}}\left(\mathrm{TeV}^{-2}\right)$ & $\Lambda^{-}(\mathrm{TeV})$ & $\Lambda^{+}(\mathrm{TeV})$ \\
\hline \hline LL & $-0.0194_{-0.0166}^{+0.0137}$ & 4.6 & 7.9 \\
\hline RR & $-0.0213_{-0.0189}^{+0.0150}$ & 4.4 & 7.6 \\
\hline VV & $-0.0127_{-0.0053}^{+0.0057}$ & 7.1 & 15.8 \\
\hline AA & $0.0029_{-0.0073}^{+0.0060}$ & 9.4 & 8.8 \\
\hline RL & $-0.1974_{-0.0220}^{+0.0678}$ & 2.1 & 7.9 \\
\hline LR & $-0.1974_{-0.0220}^{+0.0678}$ & 2.1 & 7.9 \\
\hline V0 & $-0.0134_{-0.0069}^{+0.0083}$ & 6.7 & 11.8 \\
\hline A0 & $-0.2223_{-0.0098}^{+0.0720}$ & 2.1 & 11.6 \\
\hline
\end{tabular}

\begin{tabular}{|c|c|c|c|}
\hline \multicolumn{4}{|c|}{$\boldsymbol{e}^{+} \boldsymbol{e}^{-} \rightarrow \boldsymbol{l}^{+} \boldsymbol{l}^{-}$} \\
\hline Model & $\epsilon_{-\sigma_{-}}^{+\sigma_{+}}\left(\mathrm{TeV}^{-2}\right)$ & $\Lambda^{-}(\mathrm{TeV})$ & $\Lambda^{+}(\mathrm{TeV})$ \\
\hline \hline $\mathrm{LL}$ & $-0.0017_{-0.0071}^{+0.0068}$ & 8.2 & 9.1 \\
\hline $\mathrm{RR}$ & $-0.0015_{-0.0077}^{+0.0073}$ & 7.9 & 8.7 \\
\hline $\mathrm{VV}$ & $-0.0012_{-0.0024}^{+0.0023}$ & 13.7 & 16.5 \\
\hline AA & $0.0011_{-0.0039}^{+0.0042}$ & 12.1 & 10.6 \\
\hline RL & $-0.0071_{-0.0097}^{+0.0090}$ & 6.5 & 8.7 \\
\hline LR & $-0.0071_{-0.0097}^{+0.0090}$ & 6.5 & 8.7 \\
\hline V0 & $-0.0007_{-0.0037}^{+0.0035}$ & 11.5 & 12.6 \\
\hline A0 & $-0.0035_{-0.0048}^{+0.0045}$ & 9.2 & 12.2 \\
\hline
\end{tabular}

Table 30: Fitted values of $\epsilon$ and 95\% confidence lower limits on the scale, $\Lambda$, of contact interactions in the models discussed in the text, for $\mathrm{e}^{+} \mathrm{e}^{-} \rightarrow \mathrm{e}^{+} \mathrm{e}^{-}, \mathrm{e}^{+} \mathrm{e}^{-} \rightarrow \mu^{+} \mu^{-}$, $\mathrm{e}^{+} \mathrm{e}^{-} \rightarrow \tau^{+} \tau^{-}$and $\mathrm{e}^{+} \mathrm{e}^{-} \rightarrow \mathrm{l}^{+} \mathrm{l}^{-}$, a simultaneous fit to the above, assuming lepton universality in the contact interactions. The errors on $\epsilon$ come from the statistical and systematic errors on the determination of the cross-sections and asymmetries. 
while the differential cross-section for the process $\mathrm{e}^{+} \mathrm{e}^{-} \rightarrow \mathrm{e}^{+} \mathrm{e}^{-}$can be expressed as [53]

$$
\mathrm{d} \sigma / \mathrm{d} \cos \theta=\mathrm{d} \sigma /\left.\mathrm{d} \cos \theta\right|_{S M}-C_{1}^{e}(s, t)\left[\frac{\lambda}{\mathrm{M}_{\mathrm{s}}^{4}}\right]+C_{2}^{e}(s, t)\left[\frac{\lambda}{\mathrm{M}_{\mathrm{s}}^{4}}\right]^{2},
$$

with $\theta$, as usual, being the polar angle of the outgoing fermion with respect to the direction of the incoming electron. The functions $C_{1}^{f}, C_{2}^{f}, C_{1}^{e}$ and $C_{2}^{e}$ are known $[52,53]$. For $\mathrm{e}^{+} \mathrm{e}^{-} \rightarrow \mathrm{f} \overline{\mathrm{f}}$ the expansion of $\cos \theta$ extends up to the fourth power. The dimensionless parameter $\lambda$, of $\mathcal{O}(1)$, is not explicitly calculable without full knowledge of the underlying quantum gravitational theory. It can be either positive or negative $[52,54]$. For the purposes of the fits, two cases, $\lambda= \pm 1$, are considered. This parametrisation has no explicit dependence on the number of extra dimensions, $n$. 


\begin{tabular}{|c|c|c|c|}
\hline Final State & $\begin{array}{c}\epsilon_{f i t} \\
\left(\mathrm{TeV}^{-4}\right)\end{array}$ & $\lambda$ & $\begin{array}{c}\mathrm{M}_{\mathrm{s}}(\mathrm{TeV}) \\
{[95 \% \mathrm{C} . \mathrm{L} .}\end{array}$ \\
\hline \hline $\mathrm{e}^{+} \mathrm{e}^{-}$ & $+0.54_{-0.69}^{+0.67}$ & -1 & 0.986 \\
& +1 & 0.874 \\
\hline$\mu^{+} \mu^{-}$ & $+0.84_{-1.92}^{+1.92}$ & -1 & 0.739 \\
& +1 & 0.695 \\
\hline \multirow{2}{*}{$\tau^{+} \tau^{-}$} & $-3.04_{-3.28}^{+3.28}$ & -1 & 0.578 \\
& & +1 & 0.690 \\
\hline \multirow{2}{*}{$\mathrm{l}^{+} \mathrm{l}^{-}$} & $+0.44_{-0.63}^{+0.63}$ & -1 & 0.998 \\
& & \multicolumn{3}{|c}{} & 0.898 \\
\hline
\end{tabular}

Table 31: 95\% confidence level lower limits on $\mathrm{M}_{\mathrm{s}}$ in models of gravity in extra dimensions for $\mathrm{e}^{+} \mathrm{e}^{-}, \mu^{+} \mu^{-}$and $\tau^{+} \tau^{-}$final states, and for $\mathrm{l}^{+} \mathrm{l}^{-}$, a simultaneous fit to the final states above.

\section{DELPHI}

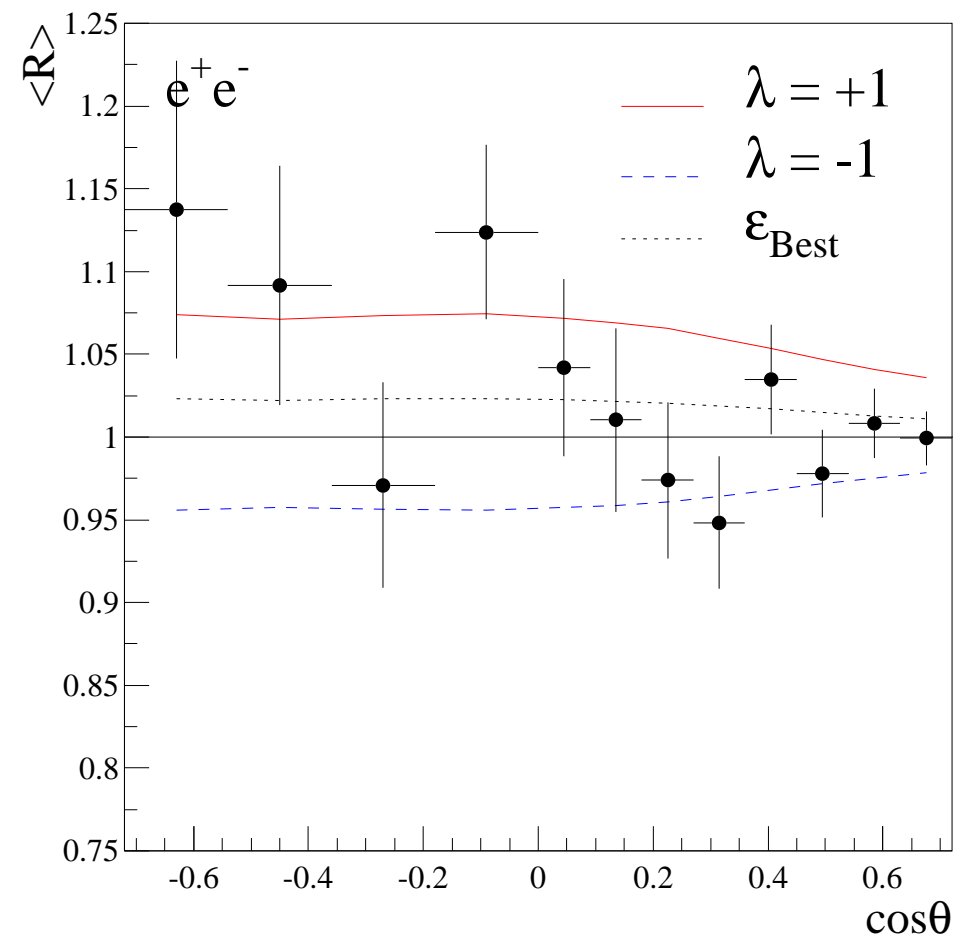

Figure 15: The deviations of the measured values of $\mathrm{d} \sigma / \mathrm{d} \cos \theta$ for $\mathrm{e}^{+} \mathrm{e}^{-} \rightarrow \mathrm{e}^{+} \mathrm{e}^{-}$from the predictions of the Standard Model, averaged over all energies. Superimposed are the predicted deviations at the luminosity weighted centre-of-mass energy for gravity in large extra dimensions for the $95 \%$ confidence limits, $\mathrm{M}_{\mathrm{s}}=0.986 \mathrm{TeV}$ for $\lambda=-1$ and $\mathrm{M}_{\mathrm{s}}=0.874$ Te $V$ for $\lambda=+1$.

\subsubsection{Results}

$\chi^{2}$ fits to the measured differential cross-sections for $\mathrm{e}^{+} \mathrm{e}^{-}, \mu^{+} \mu^{-}$and $\tau^{+} \tau^{-}$final states reported in this paper for the parameter $\epsilon=\lambda / \mathrm{M}_{\mathrm{s}}^{4}$ were performed. In order to fit the 
prediction to the data, the angular distributions given in $[52,53]$ were corrected to account for radiative effects, dominated by initial state radiation. The corrections take the form of a numerical version of the radiator function contained in ZFITTER, which are computed assuming only Standard Model processes. Separate radiator functions were derived for each angular bin in several bins of $\sqrt{s^{\prime}} / \sqrt{s}$. The tree-level new physics cross-section in a given bin was subsequently convoluted with the appropriate numerical radiator function to produce the corrected cross-section in that bin.

For the $\mu^{+} \mu^{-}$and $\tau^{+} \tau^{-}$final states the statistical errors expected from the Standard Model were used. The correlations between the systematic errors between bins of $\cos \theta$ and between channels and energies were taken into account in the fit. Errors on the parameter $\epsilon$ were determined in the same way as in Section 6.3.2.

The values of $\epsilon$ obtained are compatible with the Standard Model, i.e. $\epsilon=0 \mathrm{TeV}^{-4}$. Table 31 shows the fitted values of $\epsilon$ and $95 \%$ confidence level lower limits on $\mathrm{M}_{\mathrm{s}}$. These limits were obtained using a method equivalent to that used to extract the limits on the scale, $\Lambda$, of contact interactions, as described in Section 6.3.2.

The deviations of the measured angular distributions from the Standard Model predictions averaged over all energies in the channel $\mathrm{e}^{+} \mathrm{e}^{-} \rightarrow \mathrm{e}^{+} \mathrm{e}^{-}$are shown in Figure 15 together with the expected deviations as a function of $\cos \theta$ for the fitted best value of $\epsilon$ and for the $95 \%$ lower limits on $\mathrm{M}_{\mathrm{s}}$ for $\lambda= \pm 1$.

\subsection{Sneutrino exchange models}

These models consider possible $s$ - or $t$-channel sneutrino $\tilde{\nu}_{l}$ exchange in R-parity violating supersymmetry [55], which can affect the channel $\mathrm{e}^{+} \mathrm{e}^{-} \rightarrow \mathrm{l}^{+} \mathrm{l}^{-}$. The purely leptonic part of the R-parity violating superpotential has the form

$$
\lambda_{i j k} L_{L}^{i} L_{L}^{j} \bar{E}_{R}^{k}
$$

where $i j k$ are family indices, $L_{L}$ represents a left-handed leptonic superfield doublet and $\bar{E}_{R}$ corresponds to the right-handed singlet superfield of charged leptons. The coupling $\lambda_{i j k}$ is only non-zero for combinations involving at least two generations and for $i<j$.

For the channel $\mathrm{e}^{+} \mathrm{e}^{-} \rightarrow \mathrm{e}^{+} \mathrm{e}^{-}$there are possible contributions from the $s$-channel production and $t$-channel exchange of either $\tilde{\nu}_{\mu}\left(\lambda_{121} \neq 0\right)$ or $\tilde{\nu}_{\tau}\left(\lambda_{131} \neq 0\right)$. For the channels $\mathrm{e}^{+} \mathrm{e}^{-} \rightarrow \mu^{+} \mu^{-}$and $\mathrm{e}^{+} \mathrm{e}^{-} \rightarrow \tau^{+} \tau^{-}$there is no $s$-channel contribution if only one of the $\lambda_{i j k}$ 's is non-zero. For $\mathrm{e}^{+} \mathrm{e}^{-} \rightarrow \mu^{+} \mu^{-}$there are $t$-channel contributions from either $\tilde{\nu}_{\mathrm{e}}$ $\left(\lambda_{121} \neq 0\right), \tilde{\nu}_{\mu}\left(\lambda_{122} \neq 0\right)$ or from $\tilde{\nu}_{\tau}\left(\lambda_{132}\right.$ or $\left.\lambda_{231} \neq 0\right)$. If both $\lambda_{131} \neq 0$ and $\lambda_{232} \neq 0$ then the $s$-channel production of $\tilde{\nu}_{\tau}$ is possible. For $\mathrm{e}^{+} \mathrm{e}^{-} \rightarrow \tau^{+} \tau^{-}$there are $t$-channel contributions from either $\tilde{\nu}_{\mathrm{e}}\left(\lambda_{131} \neq 0\right), \widetilde{\nu}_{\mu}\left(\lambda_{123}\right.$ or $\left.\lambda_{231} \neq 0\right)$ or from $\tilde{\nu}_{\tau}\left(\lambda_{133} \neq 0\right)$. If both $\lambda_{121} \neq 0$ and $\lambda_{233} \neq 0$ then the $s$-channel production of $\tilde{\nu}_{\mu}$ is possible.

All these possibilities are considered here. For a given scenario the $s$ - or $t$-channel sneutrino exchange amplitude contribution is added to the Standard Model contribution as appropriate. If there is no sneutrino exchange for a specific channel then the prediction for that channel is just the SM value.

In the case of $s$-channel sneutrino graphs, if the sneutrino mass, $m_{\tilde{\nu}}$, is equal to the centre-of-mass energy of the $\mathrm{e}^{+} \mathrm{e}^{-}$beams, resonant sneutrino production occurs, which can lead to a large change in the cross-section. A lesser change in the cross-section will occur for $m_{\tilde{\nu}}<\sqrt{s}$ due to the process of radiative return. There is some sensitivity to $m_{\tilde{\nu}}$ just above $\sqrt{s}$ due to the finite width of the particle. It is assumed here that the sneutrino width is $1 \mathrm{GeV}$. 


\subsubsection{Fits to models of sneutrino exchange}

The total cross-section and forward-backward asymmetry values for the channels $\mathrm{e}^{+} \mathrm{e}^{-} \rightarrow \mathrm{e}^{+} \mathrm{e}^{-}, \mathrm{e}^{+} \mathrm{e}^{-} \rightarrow \mu^{+} \mu^{-}$and $\mathrm{e}^{+} \mathrm{e}^{-} \rightarrow \tau^{+} \tau^{-}$, at each centre-of-mass energy, were used in the fits. The theoretical prediction consisted of Improved Born Approximation Standard Model terms, plus sneutrino exchange, plus interference terms.

All the fits considered result in values of $\lambda$ which are compatible with zero; so results are expressed as $95 \%$ confidence limits. The first fits considered are to those terms which modify the $\mathrm{e}^{+} \mathrm{e}^{-} \rightarrow \mathrm{e}^{+} \mathrm{e}^{-}$channel. These involve the $s$ - and $t$-channel exchange of a $\tilde{\nu}_{\mu}($ $\left.\lambda_{121} \neq 0\right)$ or $\tilde{\nu}_{\tau}\left(\lambda_{131} \neq 0\right)$. The resulting $95 \%$ limits on $\lambda$, as a function of $m_{\tau}$, are given in Figure 16a. The best limits on $\lambda$ are obtained for the case where $m_{\tilde{\nu}}$ is close to the actual centre-of-mass energy of the LEP collisions, but the radiative return process gives some sensitivity between these points. It can be seen that $\lambda$ greater than approximately 0.05 can be excluded for $m_{\tilde{\nu}}$ in the LEP II range of energies at the $95 \%$ confidence level.

For the case that only one $\lambda$ value is non-zero there are only $t$-channel sneutrino effects for $\mathrm{e}^{+} \mathrm{e}^{-} \rightarrow \mu^{+} \mu^{-}$and $\mathrm{e}^{+} \mathrm{e}^{-} \rightarrow \tau^{+} \tau^{-}$. The values of $\lambda$ obtained for the $\mathrm{e}^{+} \mathrm{e}^{-} \rightarrow \mu^{+} \mu^{-}$ channel and for the $\mathrm{e}^{+} \mathrm{e}^{-} \rightarrow \tau^{+} \tau^{-}$channel are all consistent with zero, so results are expressed as $95 \%$ confidence limits in Table 32 .

For the fits assuming that $\lambda_{131}=\lambda_{232}=\lambda$, the resulting $95 \%$ confidence limits on $\lambda$, as a function of $m_{\tilde{\nu}}$, are given in Figure 16b. A similar exclusion pattern to that obtained from the $\mathrm{e}^{+} \mathrm{e}^{-} \rightarrow \mathrm{e}^{+} \mathrm{e}^{-}$channel is obtained. Values of $\lambda$ greater than approximately 0.07 can be excluded for $m_{\tilde{\nu}}$ in most of the LEP II range of energies at the $95 \%$ confidence level. The exclusion contour for $\lambda_{121}=\lambda_{233}=\lambda$ is shown in Figure 16c, from which it can be seen that again a similar exclusion pattern is obtained.

\section{Conclusions}

Analyses of cross-sections and forward-backward asymmetries and differential crosssections in the $\mathrm{e}^{+} \mathrm{e}^{-} \rightarrow \mathrm{e}^{+} \mathrm{e}^{-}, \mathrm{e}^{+} \mathrm{e}^{-} \rightarrow \mu^{+} \mu^{-}$and $\mathrm{e}^{+} \mathrm{e}^{-} \rightarrow \tau^{+} \tau^{-}$channels, at centre-of-mass energies from 183 to $207 \mathrm{GeV}$, have been presented, along with measurements of crosssections for $\mathrm{e}^{+} \mathrm{e}^{-} \rightarrow \mathrm{q} \overline{\mathrm{q}}$, in Sections 5.1 to 5.4. The results of the measurements are presented in Tables 5, 10, 15 and 19 for cross-sections and asymmetries and in Tables 6, 11 and 16 for differential cross-sections. Results of the measurements of cross-sections and asymmetries from previous analyses [3] at centre-of-mass energies of 130 to $172 \mathrm{GeV}$ are included in the tables.

To compare data to the predictions of the Standard Model, averages of the ratios of measurements for non-radiative samples of events to the predictions of the Standard Model have been made over all centre-of-mass energies. The results of these averages are presented in Tables 21 and 22. The precision obtained on the averaged ratios for

\begin{tabular}{|c|c|c|}
\hline coupling & $\begin{array}{c}m_{\tilde{\nu}}=100 \mathrm{GeV} / c^{2} \\
(95 \% \text { c.l. })\end{array}$ & $\begin{array}{c}m_{\tilde{\nu}}=200 \mathrm{GeV} / c^{2} \\
(95 \% \text { c.l. })\end{array}$ \\
\hline$\lambda$ (t-chann. $\widetilde{\nu}_{l}$ in $\left.\mathrm{e}^{+} \mathrm{e}^{-} \rightarrow \mu^{+} \mu^{-}\right)$ & 0.19 & 0.26 \\
$\lambda$ (t-chann. $\widetilde{\nu}_{l}$ in $\left.\mathrm{e}^{+} \mathrm{e}^{-} \rightarrow \tau^{+} \tau^{-}\right)$ & 0.56 & 0.63 \\
\hline
\end{tabular}

Table 32: Limits on the various couplings $\lambda$ in t-channel sneutrino exchange in $\mathrm{e}^{+} \mathrm{e}^{-} \rightarrow \mu^{+} \mu^{-}$and $\mathrm{e}^{+} \mathrm{e}^{-} \rightarrow \tau^{+} \tau^{-}$. 


\section{DELPHI}
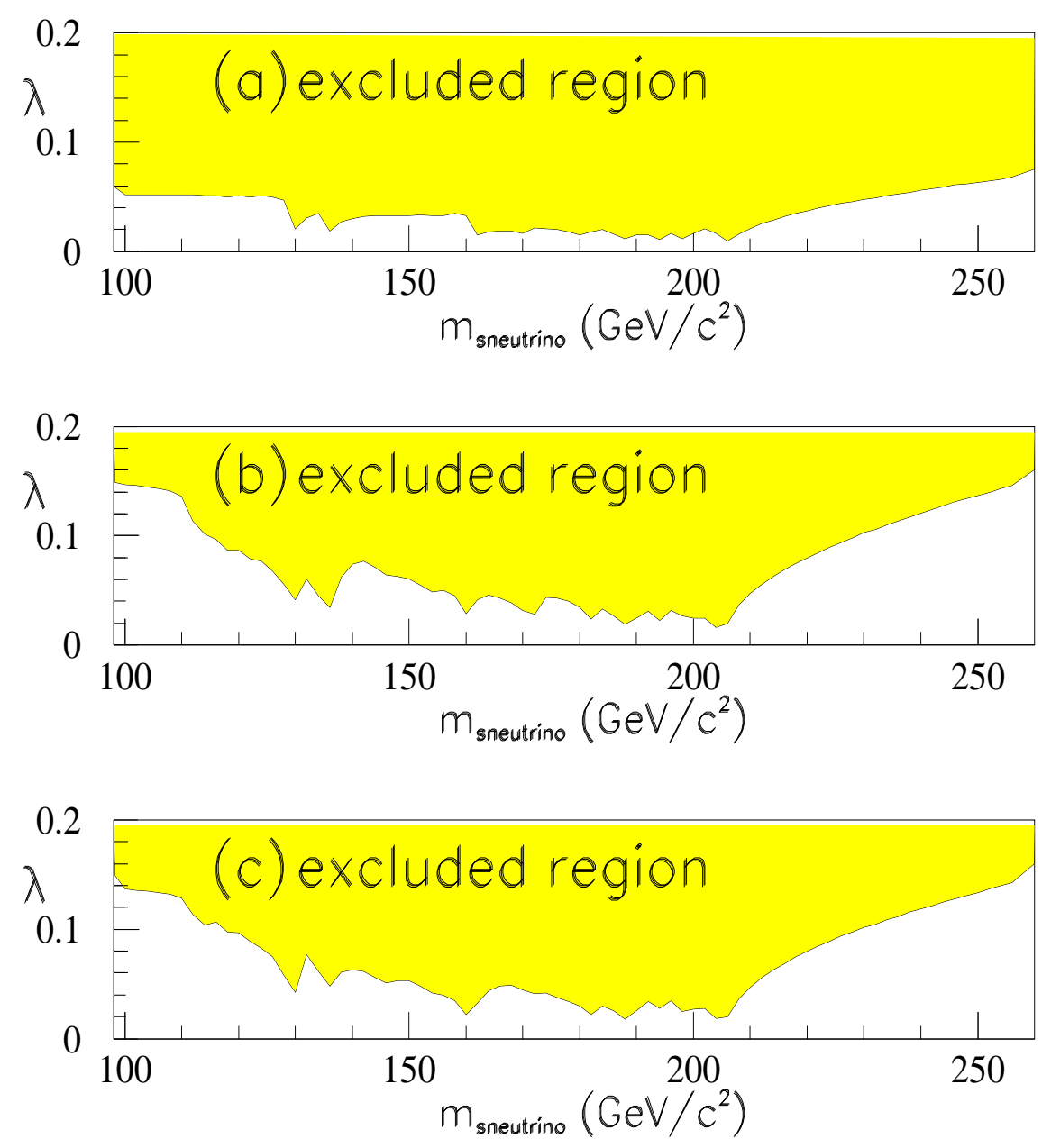

Figure 16: The $95 \%$ exclusion limits for a) $\lambda_{121}\left(\right.$ or $\left.\lambda_{131}\right)$, as a function of $m_{\sim}$ obtained from the $\mathrm{e}^{+} \mathrm{e}^{-} \rightarrow \mathrm{e}^{+} \mathrm{e}^{-}$channel; b) $\lambda_{131}=\lambda_{232}=\lambda$, as a function of $m_{\tilde{\nu}}$ obtained from the $\mathrm{e}^{+} \mathrm{e}^{-} \rightarrow \mu^{+} \mu^{-}$channel; c) $\lambda_{121}=\lambda_{233}=\lambda$, as a function of $m_{\tilde{\nu}}$ obtained from the $\mathrm{e}^{+} \mathrm{e}^{-} \rightarrow \tau^{+} \tau^{-}$channel. The sneutrino width is taken to be $1 \mathrm{GeV}$. 
the cross-sections are $\pm 2.3 \%$ for $\mathrm{e}^{+} \mathrm{e}^{-} \rightarrow \mathrm{e}^{+} \mathrm{e}^{-}$, dominated by theoretical uncertainties, $\pm 2.5 \%$ and $\pm 4.0 \%$ for $\mathrm{e}^{+} \mathrm{e}^{-} \rightarrow \mu^{+} \mu^{-}$and $\mathrm{e}^{+} \mathrm{e}^{-} \rightarrow \tau^{+} \tau^{-}$, respectively, dominated by statistical uncertainties and $\pm 1.7 \%$ for $\mathrm{e}^{+} \mathrm{e}^{-} \rightarrow \mathrm{q} \overline{\mathrm{q}}$, dominated by experimental systematic uncertainties. The results are consistent with the expectations of the Standard Model. Full details can be found in Section 5.5.

The measurements for non-radiative samples have been used to fit the parameters of the S-matrix model of $\mathrm{e}^{+} \mathrm{e}^{-} \rightarrow \mathrm{f} \overline{\mathrm{f}}$ and to search for physics beyond the Standard Model in a number of models. In all cases correlations between the experimental uncertainties have been taken into account along with theoretical uncertainties. Using LEP I data [2] alone, the best fit to the parameter $\mathrm{j}_{\text {had }}^{\text {tot }}$ of the $\mathrm{S}$-matrix Model is $0.51 \pm 0.55$. Including the LEP II measurements the best fit is $0.47 \pm 0.12$, consistent with, though somewhat above, the expectation of the Standard Model $\mathrm{j}_{\text {had }}^{\text {tot }}=0.22$. A complete set of results and description of the analysis can be found in Section 6.1. LEP I and LEP II data have also been used to search for $\mathrm{Z}^{\prime}$ bosons, yielding limits on the mass of such bosons which range from 360 to $1305 \mathrm{GeV} / c^{2}$, depending on the model assumed. Full sets of results are presented in Section 6.2.

Using measurements for non-radiative samples of leptons from the full range of centreof-mass energies, limits have been derived on the scales of contact interactions and the scale associated with the propagation of gravitons in models with large extra dimensions; these are described in Sections 6.3 and 6.4. For contact interactions the limits range from 6.5 to $16.5 \mathrm{TeV}$, assuming lepton universality. For models of gravity in large extra dimensions the limits on the scales are found to be $0.898 \mathrm{TeV}$ or $0.998 \mathrm{TeV}$, respectively, in the case when the new physics interferes either constructively or destructively with the Standard Model processes. Full details can be found in Section 6.4. In supersymmetric theories with R-parity violation, exchange of sneutrinos can affect the total and differential cross-sections for $\mathrm{e}^{+} \mathrm{e}^{-} \rightarrow \mathrm{l}^{+} \mathrm{l}^{-}$. In Section 6.5 it is shown that limits at the level of

0.05 can be set on the R-parity violating couplings for sneutrino masses in the LEP II range of energies.

\section{Acknowledgements}

We are greatly indebted to our technical collaborators, to the members of the CERNSL Division for the excellent performance of the LEP collider, and to the funding agencies for their support in building and operating the DELPHI detector.

We acknowledge in particular the support of Austrian Federal Ministry of Education, Science and Culture, GZ 616.364/2-III/2a/98, FNRS-FWO, Flanders Institute to encourage scientific and technological research in the industry (IWT), Belgium,

FINEP, CNPq, CAPES, FUJB and FAPERJ, Brazil,

Czech Ministry of Industry and Trade, GA CR 202/99/1362,

Commission of the European Communities (DG XII),

Direction des Sciences de la Matière, CEA, France,

Bundesministerium für Bildung, Wissenschaft, Forschung und Technologie, Germany,

General Secretariat for Research and Technology, Greece,

National Science Foundation (NWO) and Foundation for Research on Matter (FOM), The Netherlands,

Norwegian Research Council,

State Committee for Scientific Research, Poland, SPUB-M/CERN/PO3/DZ296/2000,

SPUB-M/CERN/PO3/DZ297/2000, 2P03B 10419 and 2P03B 69 23(2002-2004) 
FCT - Fundação para a Ciência e Tecnologia, Portugal, Vedecka grantova agentura MS SR, Slovakia, Nr. 95/5195/134, Ministry of Science and Technology of the Republic of Slovenia, CICYT, Spain, AEN99-0950 and AEN99-0761, The Swedish Research Council, Particle Physics and Astronomy Research Council, UK, Department of Energy, USA, DE-FG02-01ER41155.

EEC RTN contract HPRN-CT-00292-2002. 


\section{References}

[1] DELPHI Collaboration, P. Abreu et al., Nucl. Phys. B417 (1994) 3;

DELPHI Collaboration, P. Abreu et al., Nucl. Phys. B418 (1994) 403.

[2] DELPHI Collaboration, P. Abreu et al., Eur. Phys. J. C16 (2000) 371.

[3] DELPHI Collaboration, P. Abreu et al., Eur. Phys. J. C11 (1999) 383.

[4] DELPHI Collaboration, P. Abreu et al., Phys. Lett. B485 (2000) 45.

[5] ALEPH Collaboration, D. Buskulic et al., Phys. Lett. B378 (1996) 373; ALEPH Collaboration, R. Barate et al., Phys. Lett. B399 (1997) 329; ALEPH Collaboration, R. Barate et al., Eur. Phys. J. C12 (2000) 183.

[6] L3 Collaboration, M. Acciarri et al., Phys. Lett. B370 (1996) 195; L3 Collaboration, M. Acciarri et al., Phys. Lett. B407 (1997) 361; L3 Collaboration, M. Acciarri et al., Phys. Lett. B433 (1998) 163;

L3 Collaboration, M. Acciarri et al., Phys. Lett. B464 (1999) 135;

L3 Collaboration, M. Acciarri et al., Phys. Lett. B470 (1999) 281;

L3 Collaboration, M. Acciarri et al., Phys. Lett. B479 (2000) 101; L3 Collaboration, M. Acciarri et al., Phys. Lett. B489 (2000) 81.

[7] OPAL Collaboration, G. Alexander et al., Phys. Lett. B387 (1996) 432; OPAL Collaboration, K. Ackerstaff et al., Phys. Lett. B391 (1997) 221; OPAL Collaboration, K. Ackerstaff et al., Eur. Phys. J. C2 (1998) 441; OPAL Collaboration, G. Abbiendi et al., Eur. Phys. J. C6 (1999) 1;

OPAL Collaboration, G. Abbiendi et al., Eur. Phys. J. C13 (2000) 553; OPAL Collaboration, G. Abbiendi et al., Eur. Phys. J. C33 (2004) 173.

[8] L. Arnaudon et al., Z. Phys. C66 (1995) 45;

The LEP Energy Working Group, R. Assmann et al., Z. Phys. C66 (1995) 567;

The LEP Energy Working Group, R. Assmann et al., Eur. Phys. J. C6 (1999) 187.

[9] LEP Energy Working Group, A. Blondel et al., Eur. Phys. J. C11 (1999) 573;

LEP Energy Working Group, R. Assmann et al., Eur. Phys. J. C39 (2005) 253.

[10] DELPHI Collaboration, P. Aarnio et al., Nucl. Instr. and Meth. A303 (1991) 233.

[11] DELPHI Collaboration, P. Abreu et al., Nucl. Instr. and Meth. A378 (1996) 57.

[12] The DELPHI Silicon Tracker Group, P. Chochula et al., Nucl. Instr. and Meth. A412 (1998) 304.

[13] The DELPHI Trigger Group, A. Augustinus et al., Nucl. Instr. and Meth. A515 (2003) 782.

[14] DELPHI STIC Collaboration, S. J. Alvsvaag et al., Nucl. Instr. and Meth. A425 (1999) 106.

[15] S. Jadach et al., Comp. Phys. Comm. 102 (1997) 229.

[16] S. Jadach et al., Phys. Lett. B450 (1999) 262.

[17] S. Jadach, B.F.L. Ward and Z. Was, Comp. Phys. Comm. 130 (2000) 260.

[18] S. Jadach, W. Placzek and B.F.L. Ward, Phys. Lett. B390 (1997) 298.

[19] T. Sjöstrand et al., Comp. Phys. Comm. 135 (2001) 238.

[20] T. Sjöstrand, Comp. Phys. Comm. 82 (1994) 74.

[21] DELPHI Collaboration, P. Abreu et al., Z. Phys. C73 (1996) 11.

[22] S. Jadach, J. Kühn and Z. Was, Comp. Phys. Comm. 64 (1991) 275;

S. Jadach et al., Comp. Phys. Comm. 76 (1993) 361.

[23] E. Accomando and A. Ballestrero, Comp. Phys. Comm. 99 (1997) 270;

E. Accomando, A. Ballestrero and E. Maina, Comp. Phys. Comm. 150 (2003) 166.

[24] A. Ballestrero et al., Comp. Phys. Comm. 152 (2003) 175.

[25] F. A. Berends, P. H. Daverveldt and R. Kleiss, Comp. Phys. Comm. 40 (1986) 271. 
[26] D. Bardin et al., Comp. Phys. Comm. 133 (2001) 229.

[27] G. Montagna et al., Nucl. Phys. B401 (1993) 3;

G. Montagna et al., Comp. Phys. Comm. 76 (1993) 328.

[28] W. Beenakker, F. Berends and S. van der Marck, Nucl. Phys. B349 (1991) 323.

[29] L. Lyons et al., Nucl. Instr. and Meth. A270 (1988) 110;

A. Valassi, Nucl. Instr. and Meth. A500 (2003) 391.

[30] S. Catani et al., Phys. Lett. B269 (1991) 432.

[31] L. Lönnblad, Comp. Phys. Comm. 71 (1992) 15.

[32] M. Kobel et al., "Two-Fermion Production in Electron Positron Collisions" in S. Jadach et al. [eds], "Reports of the Working Groups on Precision Calculations for LEP2 Physics: proceedings" CERN 2000-009, hep-ph/0007180.

[33] M. Czakon, J. Gluza and J. Hejczyk, Nucl. Phys. B642 (2002) 157.

[34] A. Leike, T. Riemann and J. Rose, Phys. Lett. B273 (1991) 513;

T. Riemann, Phys. Lett. B293 (1992) 451.

[35] S. Kirsch and T. Riemann, Comp. Phys. Comm. 88 (1995) 89.

[36] A. Leike, S. Riemann and T. Riemann, hep-ph/9808374 (1991).

[37] A. Leike, S. Riemann and T. Riemann, Phys. Lett. B291 (1992) 187.

[38] P. Langacker, R. Robinett and J. Rosner, Phys. Rev. D30 (1984) 1470.

[39] D. London and J. Rosner, Phys. Rev. D34 (1986) 1530.

[40] J. Pati and A. Salam, Phys. Rev. D10 (1974) 275.

[41] R. Mohapatra and J. Pati, Phys. Rev. D11 (1975) 566.

[42] A. Leike and S. Riemann, Z. Phys. C75 (1997) 341.

[43] F. James, Comp. Phys. Comm. 20 (1980) 29.

[44] G. Altarelli, B. Mele and M. Ruiz-Altaba, Z. Phys. C45 (1989) 109; erratum Z. Phys. C47 (1990) 676.

[45] E. Eichten, K. Lane and M. Peskin, Phys. Rev. Lett. 50 (1983) 811.

[46] H. Kroha, Phys. Rev. D46 (1992) 58.

[47] ALEPH Collaboration, D. Buskulic et al., Z. Phys. C59 (1993) 215.

[48] OPAL Collaboration, G. Alexander et al., Phys. Lett. B387 (1996) 432.

[49] N. Arkani-Hamed et al., Phys. Rev. D59 (1999) 086004.

[50] C. D. Hoyle et al., Phys. Rev. D70 (2004) 042004.

[51] G. F. Giudice, T. Plehn, and A. Strumia, Nucl. Phys. B706 (2004) 455.

[52] J. L. Hewett, Phys. Rev. Lett. 82 (1999) 4765.

[53] T. G. Rizzo, Phys. Rev. D59 (1999) 115010.

[54] G. F. Giudice, R. Rattazzi and J.D. Wells, Nucl. Phys. B544 (1999) 3.

[55] J. Kalinowski et al., Phys. Lett. B406 (1997) 314. 